\title{
Hexakis( $m$-phenylene ethynylene) Macrocycles with Multiple H-Bonding Side Chains and Modified Cavities: Altered Stacking Strength and Persistent Tubular Assembly
}

\author{
Yulong Zhong, ${ }^{\dagger}$ Qiuhua Wang, ${ }^{\dagger}$ Yi Yang, ${ }^{\dagger}$ Zhonglin Lu, ${ }^{\dagger}$ Lan He, ${ }^{* \dagger}$ and Bing Gong ${ }^{* \dagger}$ \\ ${ }^{\dagger}$ College of Chemistry, Beijing Normal University, Beijing 100875, China \\ "Department of Chemistry, The State University of New York at Buffalo, Buffalo, New York 14260, \\ United States \\ $\S$ National Institute for Food and Drug Control, Beijing 100050, China
}

\section{Supporting Information}

I. General Information $\quad$ S2

II. Synthetic Procedures and Analytical Data $\quad$ S2

III. ${ }^{1} \mathrm{H}$ and ${ }^{13} \mathrm{C}$ NMR Spectra of Macrocycles 1a-l $\quad$ S37

IV. MALDI-TOF Spectra of Macrocycles 1a-I S49

V. ${ }^{1} \mathrm{H}$ NMR Spectra of Macrocycles 1a, 1c, 1g, 1i, and 1k $\quad$ S60

VI. UV Spectra of Macrocycles 1a, 1c, $1 \mathbf{g}, \mathbf{1 i}$, and 1k

VII. CD Spectra of Macrocycles 1a, 1c, 1g, 1i, and 1k S63 


\section{General information}

Chemicals were purchased from commercial sources and used as received. Silica gel foranalytical thin layer chromatography (TLC) and column chromatography (200 300 mesh) werepurchased from Qingdao Haiyang Chemical Co., Ltd \& Special Silica Gel Factory.

${ }^{1} \mathrm{H}$ NMRspectra were recorded at $400 \mathrm{MHz}$ and ${ }^{13} \mathrm{C}$ NMR spectra were measured at $100 \mathrm{MHz}$ on a BrukerAV400 spectrometer at ambient temperature. Chemical shifts were reported in parts per milliondownfield from TMS (tetramethylsilane). MS (MALDI-TOF) spectra were performed with an Autoflex instrument and an ultraflexTOF/TOF instrument. HRMS wereperformed with a micrOTOF-QII instrument. Circular dichroism spectra and UV spectra were measured on ChirascanTMfromApplied Photophysics Ltd.

\section{Synthetic Procedures and Analytical Data}

Scheme S1. Synthesis of Monomeric Building Blocks.

(a)

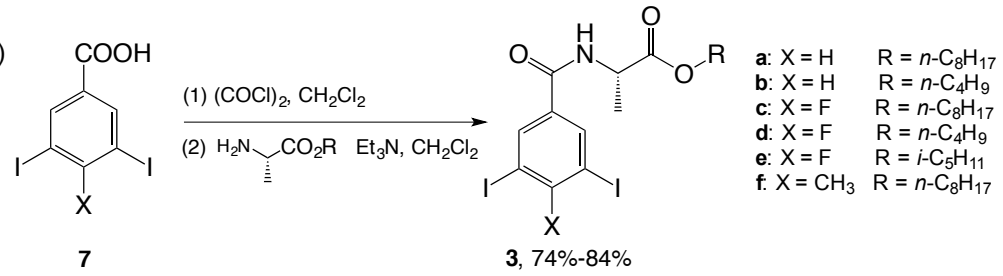

(b)

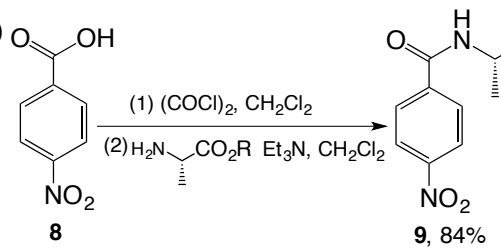

(c)
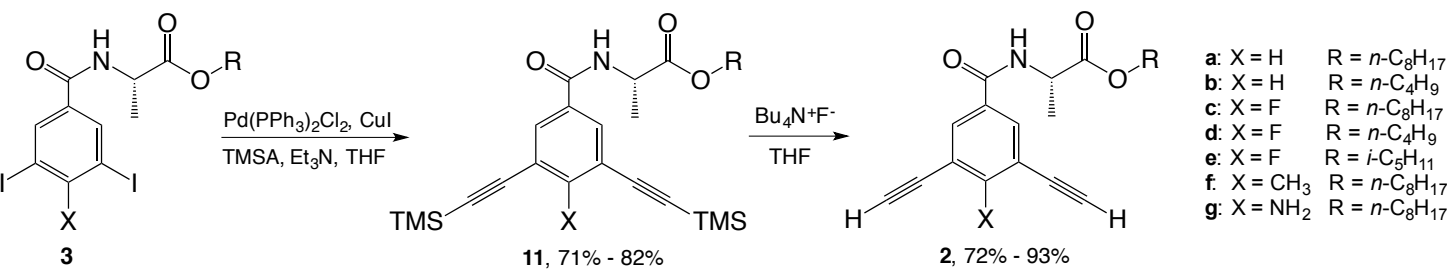
Scheme S2. Synthesis of Trimeric Precursors.

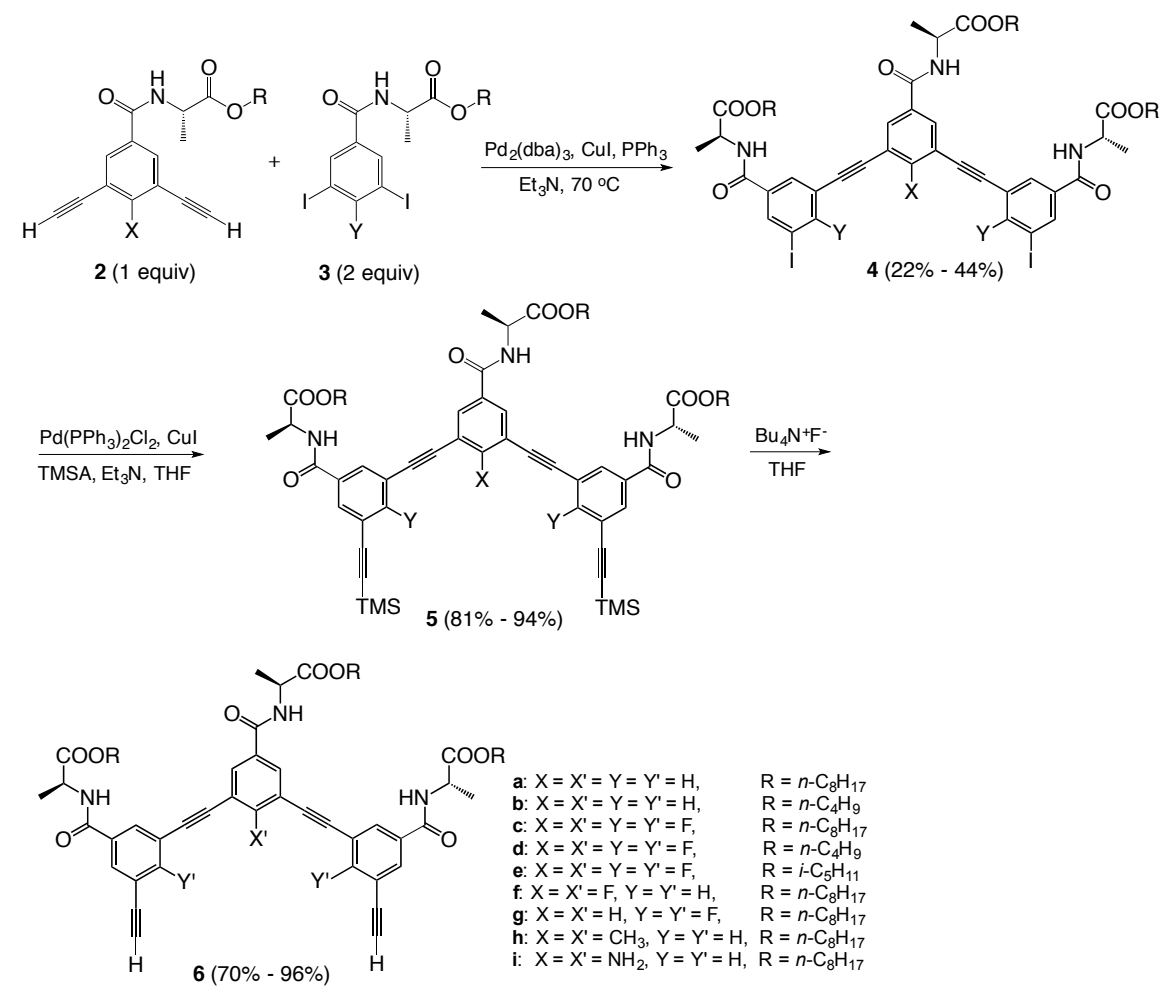

Scheme S3. Synthesis of Macrocycles 1.

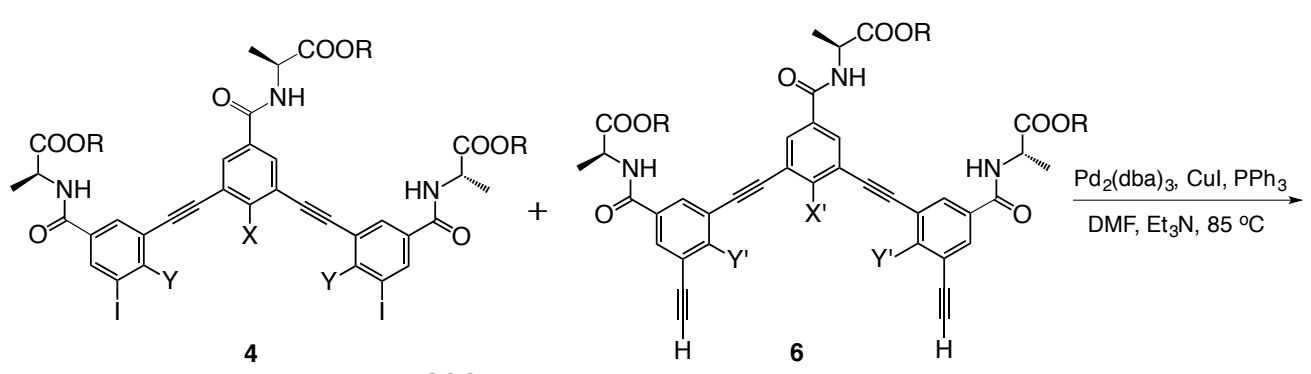

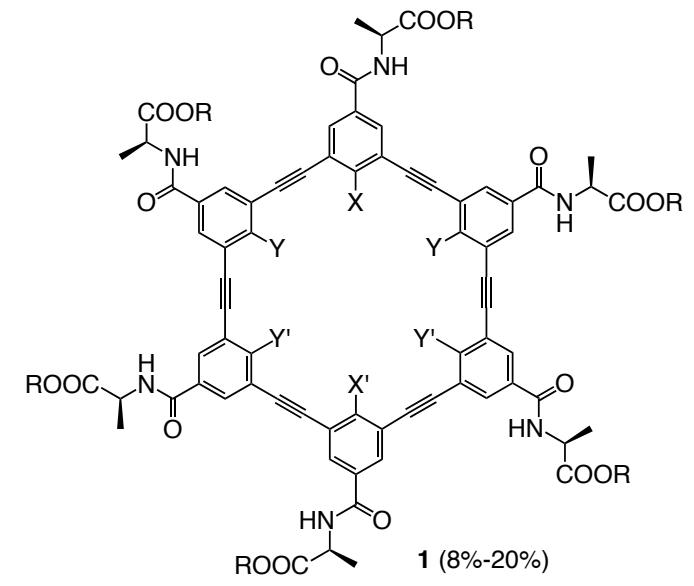

1a: $X=X^{\prime}=Y=Y^{\prime}=H$

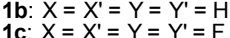

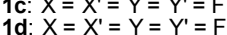

1d: $X=X^{\prime}=Y=Y^{\prime}=F$

1f: $X=Y^{\prime}=F, X^{\prime}=Y=H$

1g: $X=X^{\prime}=F, Y=Y^{\prime}=H$

1h: $X=\mathrm{CH}_{3}, \mathrm{X}^{\prime}=\mathrm{Y}=\mathrm{Y}^{\prime}=\mathrm{H}$

ii: $X=X^{\prime}=C^{\prime} H_{3}, Y=Y^{\prime}=H$

1.: $X=X^{\prime}=\mathrm{NH}_{2}, Y=Y^{\prime}=\mathrm{H}^{\prime}$

11: $X=\mathrm{NH}_{2}, X^{\prime}=F, Y=Y^{\prime}=H$

$\begin{aligned} \mathrm{R} & =n-\mathrm{C}_{8} \mathrm{H}_{17} \\ \mathrm{R} & =n-\mathrm{C}_{4} \mathrm{H}_{9} \\ \mathrm{R} & =n-\mathrm{C}_{8} \mathrm{H}_{17}\end{aligned}$

$\begin{array}{ll}\mathrm{R} & =n-\mathrm{C}_{4} \mathrm{H}_{9} \\ \mathrm{R}=n-\mathrm{C}_{8} & \mathrm{H}_{17} \\ \mathrm{R} & =n-\mathrm{C}_{4} \mathrm{H}_{9}\end{array}$

$\mathrm{R}=n-\mathrm{C}_{4} \mathrm{H}_{9}$

$\mathrm{R}=i-\mathrm{C}_{5} \mathrm{H}_{11}$

$\mathrm{R}=n-\mathrm{C}_{8} \mathrm{H}_{17}$

$\mathrm{R}=n-\mathrm{C}_{8} \mathrm{H}_{17}$

$\mathrm{R}=n-\mathrm{C}_{8} \mathrm{H}_{17}$

$\mathrm{R}=n-\mathrm{C}_{8} \mathrm{H}_{17}$

$\mathrm{R}=n-\mathrm{C}_{8} \mathrm{H}_{17}$

$\mathrm{R}=n-\mathrm{C}_{8} \mathrm{H}_{8} \mathrm{H}_{17}$

ROOC 1 (8\%-20\%) 
(S)-Octyl-2-(3,5-diiodobenzamido)propanoate (3a). To a $250 \mathrm{~mL}$ round bottom flask was added 3,5-diiodobenzoic acid (10.0 g, $26.7 \mathrm{mmol}), 100 \mathrm{~mL}$ redistilled $\mathrm{CH}_{2} \mathrm{Cl}_{2}$ and $2.5 \mathrm{~mL}(\mathrm{COCl})_{2}$ (oxalyl chloride) $(3.7 \mathrm{~g}, 29.3 \mathrm{mmol})$, then $0.1 \mathrm{~mL} \mathrm{DMF}$ was added dropwise. The solution was stirred for $6 \mathrm{~h}$. After removing the solvent and the redundant $(\mathrm{COCl})_{2}$ under reduced pressure, the residue was resolve in $20 \mathrm{~mL}$ redistilled $\mathrm{CH}_{2} \mathrm{Cl}_{2}$ and added dropwise to a $250 \mathrm{~mL}$ ice-cooled round bottom flask containing $100 \mathrm{~mL}$ redistilled $\mathrm{CH}_{2} \mathrm{Cl}_{2}, 11 \mathrm{~mL} \mathrm{Et}_{3} \mathrm{~N}(8.0 \mathrm{~g}, 79.5 \mathrm{mmol})$ and $(S)$-octyl-2-aminopropanoate hydrochloride $(6.7 \mathrm{~g}, 28.2 \mathrm{mmol})$. The mixture was stirred for $8 \mathrm{~h}$ at r.t.. The solution was washed with water and brine, and then concentrated in vacuo. The crude product was purified by column chromatography (petroleum ether/acetone $=8 / 1$ ) to afford a white solid $(11.2 \mathrm{~g}$, 75\%). ${ }^{1} \mathbf{H}$ NMR $\left(400 \mathrm{MHz}, \mathrm{CDCl}_{3}\right) \delta 8.16(\mathrm{t}, J=1.5 \mathrm{~Hz}, 1 \mathrm{H}), 8.06(\mathrm{~d}, J=1.5 \mathrm{~Hz}, 2 \mathrm{H})$, $6.90(\mathrm{~d}, J=7.3 \mathrm{~Hz}, 1 \mathrm{H}), 4.74(\mathrm{p}, J=7.2 \mathrm{~Hz}, 1 \mathrm{H}), 4.24-4.15(\mathrm{~m}, 2 \mathrm{H}), 1.72-1.64(\mathrm{~m}$, 2H), $1.51(\mathrm{~d}, J=7.1 \mathrm{~Hz}, 3 \mathrm{H}), 1.43-1.21(\mathrm{~m}, 10 \mathrm{H}), 0.90-0.86(\mathrm{~m}, 3 \mathrm{H}) .{ }^{13} \mathbf{C}$ NMR (101 $\left.\mathrm{MHz}, \mathrm{CDCl}_{3}\right) \delta 173.25,163.87,148.11,137.30,135.49,94.88,66.11,48.87,31.85,29.25$, 28.60, 25.90, 22.73, 18.59, 14.20. HRMS (ESI): calcd for $\mathrm{C}_{18} \mathrm{H}_{25} \mathrm{I}_{2} \mathrm{NO}_{3} \mathrm{Na}[\mathrm{M}+\mathrm{Na}]^{+}$ 579.9816 found 579.9787 .

(S)-Octyl-2-(3,5-bis(2-(trimethylsilyl)ethynyl)benzamido)propanoate (11a). To a 100 $\mathrm{mL}$ two-necked flask charged with 3a (5.6g, $10.0 \mathrm{mmol}), \mathrm{Pd}\left(\mathrm{PPh}_{3}\right)_{2} \mathrm{Cl}_{2}(214.5 \mathrm{mg}, 0.3$ mmol) and $\mathrm{CuI}(114.3 \mathrm{mg}, 0.6 \mathrm{mmol})$ under argon, $40 \mathrm{~mL}$ THF and $20 \mathrm{~mL} \mathrm{Et}_{3} \mathrm{~N}$ were added via syringe. The solution was stirred for $10 \mathrm{~min}$ and then $5.6 \mathrm{~mL}$ of ethynyltrimethylsilane $(3.9 \mathrm{~g}, 40 \mathrm{mmol})$ was injected into the solution. The mixture was stirred overnight at r.t.. After removing the solvent under reduced pressure, the residue was purified by column chromatography (petroleum ether/acetone $=30 / 1$ ) to afford a yellow oil (3.9g, 78\%). ${ }^{\mathbf{1}} \mathbf{H}$ NMR $\left(400 \mathrm{MHz}, \mathrm{CDCl}_{3}\right) \delta 7.57(\mathrm{~d}, J=1.5 \mathrm{~Hz}, 2 \mathrm{H}), 7.42(\mathrm{t}, J$ $=1.5 \mathrm{~Hz}, 1 \mathrm{H}), 6.68(\mathrm{~d}, J=7.3 \mathrm{~Hz}, 1 \mathrm{H}), 4.50(\mathrm{p}, J=7.1 \mathrm{~Hz}, 1 \mathrm{H}), 3.98-3.86(\mathrm{~m}, 2 \mathrm{H})$, $1.48-1.35(\mathrm{~m}, 2 \mathrm{H}), 1.26(\mathrm{~d}, J=7.1 \mathrm{~Hz}, 3 \mathrm{H}), 1.14-0.96(\mathrm{~m}, 10 \mathrm{H}), 0.66-0.61(\mathrm{~m}, 3 \mathrm{H})$, 0.00 (s, 18H). ${ }^{13} \mathrm{C}$ NMR $\left(101 \mathrm{MHz}, \mathrm{CDCl}_{3}\right) \delta 173.15,165.24,137.84,134.40,130.27$, 
124.02, 103.04, 96.24, 65.84, 48.71, 31.78, 29.17, 28.55, 25.82, 22.66, 18.59, 14.13. HRMS (ESI): calcd for $\mathrm{C}_{28} \mathrm{H}_{43} \mathrm{NO}_{3} \mathrm{Si}_{2} \mathrm{Na}[\mathrm{M}+\mathrm{Na}]^{+} 520.2674$ found 520.2656 .

(S)-Octyl-2-(3,5-diethynylbenzamido)propanoate (2a). A solution of 11a (3.0g, 6.0 $\mathrm{mmol})$ in $30 \mathrm{~mL}$ THF was added a solution of tetrabutylammonium fluoride (4.8g, 18.1 $\mathrm{mmol}$ ) in $10 \mathrm{~mL}$ THF at r.t. The mixture was stirred for $30 \mathrm{~min}$. After removing the solvent under reduced pressure, the residue was redissolved in $30 \mathrm{~mL} \mathrm{CHCl}_{3}$ and washed with water and brine. Then the organic layer was dried over anhydrous $\mathrm{Na}_{2} \mathrm{SO}_{4}$ and the solvent was removed under reduced pressure. The crude product was purified by column chromatography (petroleum ether/acetone $=10 / 1)$ to afford a white solid $(1.9 \mathrm{~g}, 90 \%) .{ }^{1} \mathbf{H}$ NMR $\left(400 \mathrm{MHz}, \mathrm{CDCl}_{3}\right) \delta 7.88(\mathrm{~d}, J=1.5 \mathrm{~Hz}, 2 \mathrm{H}), 7.71(\mathrm{t}, J=1.6 \mathrm{~Hz}, 1 \mathrm{H}), 6.79(\mathrm{~d}, J=$ $7.2 \mathrm{~Hz}, 1 \mathrm{H}), 4.76(\mathrm{t}, J=7.2 \mathrm{~Hz}, 1 \mathrm{H}), 4.25-4.10(\mathrm{~m}, 2 \mathrm{H}), 3.15(\mathrm{~s}, 2 \mathrm{H}), 1.72-1.61(\mathrm{~m}$, 2H), $1.52(\mathrm{~d}, J=7.1 \mathrm{~Hz}, 3 \mathrm{H}), 1.41-1.19(\mathrm{~m}, 10 \mathrm{H}), 0.94-0.83(\mathrm{~m}, 3 \mathrm{H}) .{ }^{13} \mathbf{C}$ NMR $(101$ $\left.\mathrm{MHz}, \mathrm{CDCl}_{3}\right) \delta 173.22,165.11,138.30,134.79,130.95,123.28,81.75,79.19,66.07$, 48.85, 31.88, 29.28, 28.64, 25.93, 22.76, 18.78, 14.22. HRMS (ESI): calcd for $\mathrm{C}_{22} \mathrm{H}_{27} \mathrm{NO}_{3} \mathrm{Na}[\mathrm{M}+\mathrm{Na}]^{+} 376.1883$ found 376.1876 .

Compound 4a. To a $100 \mathrm{~mL}$ two-necked flask charged with 3a (557.2 mg, $1.0 \mathrm{mmol})$, 2a (176.4 mg, $0.50 \mathrm{mmol}), \mathrm{Pd}_{2}(\mathrm{dba})_{3}(13.7 \mathrm{mg}, 0.015 \mathrm{mmol})$ and $\mathrm{CuI}(5.7 \mathrm{mg}, 0.030$ $\mathrm{mmol}), \mathrm{PPh}_{3}(39.8 \mathrm{mg}, 0.15 \mathrm{mmol})$ under argon, $50 \mathrm{~mL}$ degassed $\mathrm{Et}_{3} \mathrm{~N}$ was added via syringe. The mixture was stirred at $75^{\circ} \mathrm{C}$ for $12 \mathrm{~h}$, and then removed the solvent under reduced pressure. The residue was purified by column chromatography $\left(\mathrm{CHCl}_{3} / \mathrm{THF}=\right.$ 50/1) to afford a light yellow solid (143.0 mg, 24\%). ${ }^{1} \mathbf{H}$ NMR (400 MHz, $\left.\mathrm{CDCl}_{3}\right) \delta 8.13$ (d, $J=1.7 \mathrm{~Hz}, 2 \mathrm{H}), 7.99$ (d, $J=1.6 \mathrm{~Hz}, 2 \mathrm{H}), 7.92(\mathrm{~d}, J=1.5 \mathrm{~Hz}, 2 \mathrm{H}), 7.88(\mathrm{~d}, J=1.6 \mathrm{~Hz}$, 2H), $7.76(\mathrm{~d}, J=1.6 \mathrm{~Hz}, 1 \mathrm{H}), 7.13$ (d, $J=7.3 \mathrm{~Hz}, 1 \mathrm{H}), 7.02(\mathrm{~d}, J=7.3 \mathrm{~Hz}, 2 \mathrm{H}), 4.89-$ $4.75(\mathrm{~m}, 3 \mathrm{H}), 4.27-4.13(\mathrm{~m}, 6 \mathrm{H}), 1.70-1.64(\mathrm{~m}, 6 \mathrm{H}), 1.55(\mathrm{t}, J=6.8 \mathrm{~Hz}, 9 \mathrm{H}), 1.35-$ $1.25(\mathrm{~m}, 30 \mathrm{H}), 0.88(\mathrm{t}, J=6.7 \mathrm{~Hz}, 9 \mathrm{H}) .{ }^{13} \mathbf{C}$ NMR $\left(101 \mathrm{MHz}, \mathrm{CDCl}_{3}\right) \delta 173.43,173.33$, $165.12,164.57,143.04,137.25,136.56,135.95,134.91,130.56,129.53,125.00,123.69$, $93.98,89.76,88.58,66.12,48.91,31.90,30.46,29.84,29.30,28.67,25.95,22.77,18.74$, 
18.72, 14.23. MS (MALDI-TOF): calcd for $\mathrm{C}_{58} \mathrm{H}_{75} \mathrm{I}_{2} \mathrm{~N}_{3} \mathrm{O}_{9} \mathrm{Na}[\mathrm{M}+\mathrm{Na}]^{+} 1234.3485$ found 1234.8787.

Compound 5a. To a $50 \mathrm{~mL}$ two-necked flask charged with compound 4a (242.4 mg, $0.20 \mathrm{mmol}), \mathrm{Pd}\left(\mathrm{PPh}_{3}\right)_{2} \mathrm{Cl}_{2}(4.3 \mathrm{mg}, 0.006 \mathrm{mmol})$ and $\mathrm{CuI}(2.3 \mathrm{mg}, 0.012 \mathrm{mmol})$ under argon, $15 \mathrm{~mL}$ THF and $8 \mathrm{~mL} \mathrm{Et}_{3} \mathrm{~N}$ were added via syringe. The solution was stirred for $10 \mathrm{~min}$ and then $0.11 \mathrm{mLethynyltrimethylsilane}(78.4 \mathrm{mg}, 0.8 \mathrm{mmol})$ was injected into the solution. The mixture was stirred overnight at r.t.. After removing the solvent under reduced pressure, the residue was purified by column chromatography $\left(\mathrm{CHCl}_{3} / \mathrm{THF}=\right.$ 80/1) to afford a yellow oil (203.2 mg, 88\%). ${ }^{\mathbf{1}} \mathbf{H}$ NMR (400 MHz, $\left.\mathrm{CDCl}_{3}\right) \delta 7.95$ (d, J = $1.5 \mathrm{~Hz}, 2 \mathrm{H}), 7.88$ (dt, J = 9.1, $1.7 \mathrm{~Hz}, 4 \mathrm{H}), 7.74-7.68(\mathrm{~m}, 3 \mathrm{H}), 7.40$ (d, J = 7.4 Hz, 1H), $7.20(\mathrm{~d}, \mathrm{~J}=7.3 \mathrm{~Hz}, 2 \mathrm{H}), 4.92-4.74(\mathrm{~m}, 3 \mathrm{H}), 4.24-4.09(\mathrm{~m}, 6 \mathrm{H}), 1.70-1.59(\mathrm{~m}, 6 \mathrm{H})$, $1.54(\mathrm{t}, \mathrm{J}=7.1 \mathrm{~Hz}, 9 \mathrm{H}), 1.39-1.17(\mathrm{~m}, 30 \mathrm{H}), 0.92-0.79(\mathrm{~m}, 9 \mathrm{H}), 0.25(\mathrm{~s}, 18 \mathrm{H}) .{ }^{13} \mathrm{C}$ NMR $\left(101 \mathrm{MHz}, \mathrm{CDCl}_{3}\right) \delta 173.45,173.35,165.32,165.25,137.52,137.05,134.63$, $134.50,130.76,130.52,130.18,124.25,123.70,123.42,102.99,96.54,89.23,89.00$, $65.94,48.81,31.83,29.22,28.58,25.87,22.70,18.55,18.49,14.17$. MS (MALDI-TOF): calcd for $\mathrm{C}_{68} \mathrm{H}_{93} \mathrm{~N}_{3} \mathrm{O}_{9} \mathrm{Si}_{2} \mathrm{Na}[\mathrm{M}+\mathrm{Na}]^{+} 1174.6343$ found 1174.6947 .

Compound 6a. A solution of compound 5a (173.0 mg, $0.15 \mathrm{mmol})$ in $10 \mathrm{~mL}$ THF was added a solution of tetrabutylammonium fluoride $(117.5 \mathrm{mg}, 0.45 \mathrm{mmol})$ in $3 \mathrm{~mL}$ THF at r.t.. The mixture was stirred for 30min. After removing the solvent under reduced pressure, the residue was redissolved in $15 \mathrm{~mL} \mathrm{CHCl}_{3}$ and washed with water and brine. Then the organic layer was dried over anhydrous $\mathrm{Na}_{2} \mathrm{SO}_{4}$ and the solvent was removed under reduced pressure. The crude product was purified by column chromatography $\left(\mathrm{CHCl}_{3} / \mathrm{THF}=30 / 1\right)$ to afford a light yellow solid $(144.7 \mathrm{mg}, 96 \%) .{ }^{1} \mathbf{H}$ NMR $(400 \mathrm{MHz}$, $\left.\mathrm{CDCl}_{3}\right) \delta 7.94(\mathrm{~d}, J=1.5 \mathrm{~Hz}, 2 \mathrm{H}), 7.92(\mathrm{t}, J=1.5 \mathrm{~Hz}, 2 \mathrm{H}), 7.90(\mathrm{t}, J=1.5 \mathrm{~Hz}, 2 \mathrm{H}), 7.78$ (t, $J=1.5 \mathrm{~Hz}, 1 \mathrm{H}), 7.75(\mathrm{t}, J=1.4 \mathrm{~Hz}, 2 \mathrm{H}), 7.10(\mathrm{~d}, J=7.3 \mathrm{~Hz}, 1 \mathrm{H}), 6.99$ (d, $J=7.3 \mathrm{~Hz}$, 2H), $4.88-4.76(\mathrm{~m}, 3 \mathrm{H}), 4.25-4.13(\mathrm{~m}, 6 \mathrm{H}), 3.16(\mathrm{~s}, 2 \mathrm{H}), 1.71-1.62(\mathrm{~m}, 6 \mathrm{H}), 1.56(\mathrm{~m}$, 9H), $1.38-1.21(\mathrm{~m}, 30 \mathrm{H}), 0.88(\mathrm{t}, J=6.6 \mathrm{~Hz}, 9 \mathrm{H}) .{ }^{13} \mathbf{C} \mathbf{N M R}\left(101 \mathrm{MHz}, \mathrm{CDCl}_{3}\right)$ 
$\delta 173.26,173.19,165.07,137.67,137.14,134.76,134.72,130.85,130.40,123.67,123.57$, $123.28,89.10,81.72,79.07,65.94,48.76,31.76,29.16,28.54,25.82,22.63,18.60,14.09$. MS (MALDI-TOF): calcd for $\mathrm{C}_{62} \mathrm{H}_{77} \mathrm{~N}_{3} \mathrm{O}_{9} \mathrm{Na}[\mathrm{M}+\mathrm{Na}]^{+} 1030.5552$ found 1030.5910 .

Macrocycle1a. To a $100 \mathrm{~mL}$ two-necked flask charged with $\operatorname{Pd}_{2}(\mathrm{dba})_{3}(22.6 \mathrm{mg}, 0.025$ $\mathrm{mmol})$ and $\mathrm{CuI}(9.4 \mathrm{mg}, 0.049 \mathrm{mmol}), \mathrm{PPh}_{3}(65.6 \mathrm{mg}, 0.25 \mathrm{mmol})$ under argon, $20 \mathrm{~mL}$ degassed $\mathrm{Et}_{3} \mathrm{~N}$ and $40 \mathrm{~mL}$ degassed DMF were added via syringe. The solution was stirred at $85^{\circ} \mathrm{C}$ for $10 \mathrm{~min}$. A solution of compound $4 \mathrm{a}(60.6 \mathrm{mg}, 0.050 \mathrm{mmol})$ and compound 6a (49.9 mg, $0.050 \mathrm{mmol})$ in $10 \mathrm{~mL}$ DMF was slowly injected into the flask within $48 \mathrm{~h}$ by a syringe pump and then the solution was stirred for further $12 \mathrm{~h}$. The reaction mixture was concentrated in vacuo and purified by column chromatography $\left(\mathrm{CHCl}_{3} / \mathrm{THF} / \mathrm{AcOH}=25 / 1 / 0.5\right)$ to afford the crude product. The crude product was then washed with a few acetone and methanol to give the desired product $(19.5 \mathrm{mg}, 20 \%)$ as a white solid. ${ }^{1}$ H NMR (400 MHz, DMSO- $\left.d_{6}\right) \delta 9.05(\mathrm{~d}, J=6.3 \mathrm{~Hz}, 6 \mathrm{H}), 8.14(\mathrm{~s}, 12 \mathrm{H})$, $7.95(\mathrm{~s}, 6 \mathrm{H}), 4.56-4.45(\mathrm{~m}, 6 \mathrm{H}), 4.16-3.99(\mathrm{~m}, 12 \mathrm{H}), 1.57$ (m, 12H), $1.46(\mathrm{~d}, J=7.2$ $\mathrm{Hz}, 18 \mathrm{H}), 1.35-0.98(\mathrm{~m}, 60 \mathrm{H}), 0.82(\mathrm{t}, J=6.8 \mathrm{~Hz}, 18 \mathrm{H}) .{ }^{13} \mathbf{C}$ NMR $(101 \mathrm{MHz}$, DMSO- $\left.d_{6}\right) \delta 172.21,164.30,136.54,134.62,130.31,122.56,88.81,64.28,48.44,31.09$, 28.54, 28.04, 25.25, 21.97, 16.49, 13.71. MS (MALDI-TOF): calcd for $\mathrm{C}_{120} \mathrm{H}_{150} \mathrm{~N}_{6} \mathrm{O}_{18} \mathrm{Na}$ $[\mathrm{M}+\mathrm{Na}]^{+} 1986.0899$ found 1986.0107.

(S)-Butyl-2-(3,5-diiodobenzamido)propanoate (3b). To a $250 \mathrm{~mL}$ round bottom flask was added 3,5-diiodobenzoic acid (10.0 g, $26.7 \mathrm{mmol}), 100 \mathrm{~mL}$ redistilled $\mathrm{CH}_{2} \mathrm{Cl}_{2}$ and $2.5 \mathrm{~mL}(\mathrm{COCl})_{2}$ (oxalyl chloride) $(3.7 \mathrm{~g}, 29.3 \mathrm{mmol})$, then $0.1 \mathrm{~mL}$ DMF was added dropwise. The solution was stirred for $6 \mathrm{~h}$. After removing the solvent and the redundant $(\mathrm{COCl})_{2}$ under reduced pressure, the residue was resolve in $20 \mathrm{~mL}$ redistilled $\mathrm{CH}_{2} \mathrm{Cl}_{2}$ and added dropwise to a $250 \mathrm{~mL}$ ice-cooled round bottom flask containing $100 \mathrm{~mL}$ redistilled $\mathrm{CH}_{2} \mathrm{Cl}_{2}, 11 \mathrm{~mL} \mathrm{Et}_{3} \mathrm{~N}(8.0 \mathrm{~g}, 79.5 \mathrm{mmol})$ and $(S)$-butyl-2-aminopropanoate hydrochloride $(5.1 \mathrm{~g}, 28.1 \mathrm{mmol})$. The mixture was stirred for $8 \mathrm{~h}$ at r.t.. The solution was washed with water and brine, and then concentrated in vacuo. The crude product was purified by 
column chromatography (petroleum ether/acetone $=6 / 1)$ to afford a white solid $(9.9 \mathrm{~g}$, 74\%). ${ }^{1}$ H NMR $\left(400 \mathrm{MHz}, \mathrm{CDCl}_{3}\right) \delta 8.17(\mathrm{t}, J=1.5 \mathrm{~Hz}, 1 \mathrm{H}), 8.06(\mathrm{~d}, J=1.5 \mathrm{~Hz}, 2 \mathrm{H})$, $6.76(\mathrm{~d}, J=7.0 \mathrm{~Hz}, 1 \mathrm{H}), 4.74(\mathrm{~m}, 1 \mathrm{H}), 4.22-4.18(\mathrm{~m}, 2 \mathrm{H}), 1.66(\mathrm{~m}, 2 \mathrm{H}), 1.51(\mathrm{~d}, J=7.1$ $\mathrm{Hz}, 3 \mathrm{H}), 1.43-1.37(\mathrm{~m}, 2 \mathrm{H}), 0.95(\mathrm{~m}, 3 \mathrm{H}) .{ }^{\mathbf{1 3}} \mathbf{C} \mathbf{N M R}\left(101 \mathrm{MHz}, \mathrm{CDCl}_{3}\right) \delta 173.09$, $163.73,148.05,137.24,135.34,94.80,65.71,48.75,30.51,19.04,18.57,13.68$. HRMS (ESI): calcd for $\mathrm{C}_{14} \mathrm{H}_{17} \mathrm{I}_{2} \mathrm{NO}_{3} \mathrm{Na}[\mathrm{M}+\mathrm{Na}]^{+} 523.9190$ found 523.9186 .

(S)-Butyl-2-(3,5-bis(2-(trimethylsilyl)ethynyl)benzamido)propanoate(11b). To a 100 $\mathrm{mL}$ two-necked flask charged with $3 \mathbf{b}(5.0 \mathrm{~g}, 10.0 \mathrm{mmol}), \mathrm{Pd}\left(\mathrm{PPh}_{3}\right)_{2} \mathrm{Cl}_{2}(214.5 \mathrm{mg}, 0.3$ mmol) and $\mathrm{CuI}(114.3 \mathrm{mg}, 0.6 \mathrm{mmol})$ under argon, $40 \mathrm{~mL}$ THF and $20 \mathrm{~mL} \mathrm{Et}_{3} \mathrm{~N}$ were added via syringe. The solution was stirred for $10 \mathrm{~min}$ and then 5.6 mLethynyltrimethylsilane $(3.9 \mathrm{~g}, 40 \mathrm{mmol})$ was injected into the solution. The mixture was stirred overnight at r.t.. After removing the solvent under reduced pressure, the residue was purified by column chromatography (petroleum ether/acetone $=30 / 1$ ) to afford a yellow oil (3.5g, 79\%). ${ }^{1} \mathbf{H}$ NMR $\left(400 \mathrm{MHz}, \mathrm{CDCl}_{3}\right) \delta 7.74(\mathrm{~d}, J=1.5 \mathrm{~Hz}, 2 \mathrm{H})$, $7.60(\mathrm{~s}, 1 \mathrm{H}), 6.72(\mathrm{~d}, J=7.2 \mathrm{~Hz}, 1 \mathrm{H}), 4.68(\mathrm{~m}, 1 \mathrm{H}), 4.11(\mathrm{~m}, 2 \mathrm{H}), 1.62-1.54(\mathrm{~m}, 2 \mathrm{H})$, $1.43(\mathrm{~m}, 3 \mathrm{H}), 1.34-1.28(\mathrm{~m}, 2 \mathrm{H}), 0.87$ (t, $J=7.4 \mathrm{~Hz}, 3 \mathrm{H}), 0.17$ (s, 18H). ${ }^{13} \mathbf{C}$ NMR (101 $\left.\mathrm{MHz}, \mathrm{CDCl}_{3}\right) \delta 173.35,165.38,138.04,134.57,130.40,124.22,103.17,96.48,65.78$, 48.87, 30.74, 27.11, 19.25, 18.86, 14.33, 13.86, 1.22. HRMS (ESI): calcd for $\mathrm{C}_{24} \mathrm{H}_{35} \mathrm{NO}_{3} \mathrm{Si}_{2} \mathrm{Na}[\mathrm{M}+\mathrm{Na}]^{+} 464.2048$ found 464.2021 .

(S)-Butyl-2-(3,5-diethynylbenzamido)propanoate(2b). A solution of $\mathbf{1 1 b}$ (2.6g, 5.9 mmol) in $30 \mathrm{~mL}$ THF was added a solution of tetrabutylammoniumfluoride $(4.6 \mathrm{~g}, 17.7$ mmol) in $10 \mathrm{~mL}$ THF at r.t.. The mixture was stirred for 30min. After removing the solvent under reduced pressure, the residue was redissolved in $30 \mathrm{~mL} \mathrm{CHCl}_{3}$ and washed with water and brine. Then the organic layer was dried over anhydrous $\mathrm{Na}_{2} \mathrm{SO}_{4}$ and the solvent was removed under reduced pressure. The crude product was purified by column chromatography $($ petroleum ether/acetone $=10 / 1)$ to afford a white solid $(1.6 \mathrm{~g}, 91 \%) .{ }^{1} \mathbf{H}$ NMR (400 MHz, $\left.\mathrm{CDCl}_{3}\right) \delta 7.88(\mathrm{~d}, J=1.4 \mathrm{~Hz}, 2 \mathrm{H}), 7.72(\mathrm{~d}, J=1.3 \mathrm{~Hz}, 1 \mathrm{H}), 6.76(\mathrm{~d}, J=$ 
$7.0 \mathrm{~Hz}, 1 \mathrm{H}), 4.76(\mathrm{~m}, 1 \mathrm{H}), 4.25-4.14(\mathrm{~m}, 2 \mathrm{H}), 3.15(\mathrm{~s}, 2 \mathrm{H}), 1.67(\mathrm{~m}, 2 \mathrm{H}), 1.52(\mathrm{~d}, J=$ $7.1 \mathrm{~Hz}, 3 \mathrm{H}), 1.40(\mathrm{~m}, 2 \mathrm{H}), 0.95(\mathrm{t}, J=7.4 \mathrm{~Hz}, 3 \mathrm{H}) .{ }^{13} \mathbf{C} \mathbf{~ N M R}\left(101 \mathrm{MHz}, \mathrm{CDCl}_{3}\right) \delta$ $173.10,164.98,138.18,134.67,130.82,123.16,81.62,79.06,65.66,48.71,30.54,19.05$, 18.68, 13.67. HRMS (ESI): calcd for $\mathrm{C}_{18} \mathrm{H}_{19} \mathrm{NO}_{3} \mathrm{Na}[\mathrm{M}+\mathrm{Na}]^{+} 320.1257$ found 320.1265 .

Compound 4b. To a $100 \mathrm{~mL}$ two-necked flask charged with $\mathbf{3 b}(501.0 \mathrm{mg}, 1.0 \mathrm{mmol})$, 2b (148.5 mg, $0.50 \mathrm{mmol}), \mathrm{Pd}_{2}(\mathrm{dba})_{3}(13.7 \mathrm{mg}, 0.015 \mathrm{mmol})$ and $\mathrm{CuI}(5.7 \mathrm{mg}, 0.030$ $\mathrm{mmol}), \mathrm{PPh}_{3}(39.8 \mathrm{mg}, 0.15 \mathrm{mmol})$ under argon, $50 \mathrm{~mL}$ degassed $\mathrm{Et}_{3} \mathrm{~N}$ was added via syringe. The mixture was stirred at $75^{\circ} \mathrm{C}$ for $12 \mathrm{~h}$, and then removed the solvent under reduced pressure. The residue was purified by column chromatography $\left(\mathrm{CHCl}_{3} / \mathrm{THF}=\right.$ 40/1) to afford a light yellow solid (207.4 mg, 40\%). ${ }^{1} \mathbf{H}$ NMR (400 MHz, $\left.\mathrm{CDCl}_{3}\right) \delta 8.13$ (t, $J=1.6 \mathrm{~Hz}, 2 \mathrm{H}), 7.99$ (t, $J=1.5 \mathrm{~Hz}, 2 \mathrm{H}), 7.92$ (d, $J=1.5 \mathrm{~Hz}, 2 \mathrm{H}), 7.88$ (t, $J=1.4 \mathrm{~Hz}$, 2H), $7.77(\mathrm{~d}, J=1.5 \mathrm{~Hz}, 1 \mathrm{H}), 7.08(\mathrm{~d}, J=7.3 \mathrm{~Hz}, 1 \mathrm{H}), 6.98(\mathrm{~d}, J=7.3 \mathrm{~Hz}, 2 \mathrm{H}), 4.81(\mathrm{~m}$, $3 \mathrm{H}), 4.28-4.14(\mathrm{~m}, 6 \mathrm{H}), 1.72-1.62(\mathrm{~m}, 6 \mathrm{H}), 1.55(\mathrm{~m}, 9 \mathrm{H}), 1.41$ (m, 6H), $0.95(\mathrm{t}, J=$ $7.4 \mathrm{~Hz}, 9 \mathrm{H}) .{ }^{13} \mathbf{C}$ NMR $\left(101 \mathrm{MHz}, \mathrm{CDCl}_{3}\right) \delta 173.45,173.31,165.06,164.51,142.80$, $137.00,136.45,135.65,134.56,130.50,129.48,124.78,123.46,93.79,89.60,88.40$, $65.65,48.75,30.55,29.69,19.06,18.43,13.69$. MS (MALDI-TOF): calcd for $\mathrm{C}_{46} \mathrm{H}_{51} \mathrm{I}_{2} \mathrm{~N}_{3} \mathrm{O}_{9} \mathrm{Na}[\mathrm{M}+\mathrm{Na}]^{+} 1066.1607$ found 1066.3543 .

Compound 5b. To a $50 \mathrm{~mL}$ two-necked flask charged with compound $\mathbf{4 b}(208.1 \mathrm{mg}$, $0.20 \mathrm{mmol}), \mathrm{Pd}\left(\mathrm{PPh}_{3}\right)_{2} \mathrm{Cl}_{2}(4.3 \mathrm{mg}, 0.006 \mathrm{mmol})$ and $\mathrm{CuI}(2.3 \mathrm{mg}, 0.012 \mathrm{mmol})$ under argon, $15 \mathrm{~mL}$ THF and $8 \mathrm{~mL} \mathrm{Et}_{3} \mathrm{~N}$ were added via syringe. The solution was stirred for $10 \mathrm{~min}$ and then $0.11 \mathrm{mLethynyltrimethylsilane} \mathrm{(} 78.4 \mathrm{mg}, 0.8 \mathrm{mmol})$ was injected into the solution. The mixture was stirred overnight at r.t.. After removing the solvent under reduced pressure, the residue was purified by column chromatography $\left(\mathrm{CHCl}_{3} / \mathrm{THF}=\right.$ 80/1) to afford a yellow oil (185.2 mg, 94\%). ${ }^{1} \mathbf{H}$ NMR (400 MHz, $\left.\mathrm{CDCl}_{3}\right) \delta 7.87(\mathrm{~d}, J=$ $1.4 \mathrm{~Hz}, 2 \mathrm{H}), 7.82(\mathrm{t}, J=1.5 \mathrm{~Hz}, 2 \mathrm{H}), 7.80(\mathrm{t}, J=1.5 \mathrm{~Hz}, 2 \mathrm{H}), 7.67$ (t, $J=1.4 \mathrm{~Hz}, 1 \mathrm{H})$, $7.65(\mathrm{t}, J=1.4 \mathrm{~Hz}, 2 \mathrm{H}), 7.21(\mathrm{~d}, J=5.9 \mathrm{~Hz}, 1 \mathrm{H}), 7.03(\mathrm{~d}, J=7.3 \mathrm{~Hz}, 2 \mathrm{H}), 4.75(\mathrm{~m}, 3 \mathrm{H})$, $4.18-4.06(\mathrm{~m}, 6 \mathrm{H}), 1.58(\mathrm{~m}, 6 \mathrm{H}), 1.48(\mathrm{~m}, 9 \mathrm{H}), 1.31(\mathrm{~m}, 6 \mathrm{H}), 0.87$ (t, $J=7.4 \mathrm{~Hz}, 9 \mathrm{H})$, 
0.19 (s, 18H). ${ }^{13} \mathbf{C}$ NMR $\left(101 \mathrm{MHz}, \mathrm{CDCl}_{3}\right) \delta 173.50,173.43,165.42,137.67,137.22$, $134.83,134.68,130.80,130.57,130.28,124.39,123.85,123.57,103.09,96.71,89.37$, 89.12, 65.77, 48.91, 30.73, 19.23, 18.72, 13.86, 1.21. MS (MALDI-TOF): calcd for $\mathrm{C}_{56} \mathrm{H}_{69} \mathrm{~N}_{3} \mathrm{O}_{9} \mathrm{Si}_{2} \mathrm{Na}[\mathrm{M}+\mathrm{Na}]^{+} 1006.4465$ found 1006.6065 .

Compound 6b. A solution of compound $\mathbf{5 b}(147.6 \mathrm{mg}, 0.15 \mathrm{mmol})$ in $10 \mathrm{~mL}$ THF was added a solution of tetrabutylammonium fluoride $(117.5 \mathrm{mg}, 0.45 \mathrm{mmol})$ in $3 \mathrm{~mL}$ THF at r.t.. The mixture was stirred for 30min. After removing the solvent under reduced pressure, the residue was redissolved in $15 \mathrm{~mL} \mathrm{CHCl}_{3}$ and washed with water and brine. Then the organic layer was dried over anhydrous $\mathrm{Na}_{2} \mathrm{SO}_{4}$ and the solvent was removed under reduced pressure. The crude product was purified by column chromatography $\left(\mathrm{CHCl}_{3} / \mathrm{THF}=30 / 1\right)$ to afford alight yellow solid $(117.7 \mathrm{mg}, 93 \%) .{ }^{\mathbf{1}} \mathbf{H} \mathbf{~ N M R}(400 \mathrm{MHz}$, $\left.\mathrm{CDCl}_{3}\right) \delta 7.94(\mathrm{~d}, J=1.5 \mathrm{~Hz}, 2 \mathrm{H}), 7.93(\mathrm{t}, J=1.6 \mathrm{~Hz}, 2 \mathrm{H}), 7.90(\mathrm{t}, J=1.5 \mathrm{~Hz}, 2 \mathrm{H}), 7.81$ $(\mathrm{t}, J=1.5 \mathrm{~Hz}, 1 \mathrm{H}), 7.77(\mathrm{t}, J=1.4 \mathrm{~Hz}, 2 \mathrm{H}), 6.93(\mathrm{~d}, J=7.2 \mathrm{~Hz}, 1 \mathrm{H}), 6.86(\mathrm{~d}, J=7.2 \mathrm{~Hz}$, $2 \mathrm{H}), 4.88-4.73(\mathrm{~m}, 3 \mathrm{H}), 4.30-4.14(\mathrm{~m}, 6 \mathrm{H}), 3.17(\mathrm{~s}, 2 \mathrm{H}), 1.72-1.63(\mathrm{~m}, 6 \mathrm{H}), 1.55(\mathrm{~m}$, 9H), $1.41(\mathrm{~m}, 6 \mathrm{H}), 0.95(\mathrm{t}, J=7.4 \mathrm{~Hz}, 9 \mathrm{H}) .{ }^{13} \mathbf{C}$ NMR (101 MHz, $\left.\mathrm{CDCl}_{3}\right) \delta 173.29$, $173.21,165.09,137.65,137.12,134.71,134.68,130.86,130.39,123.64,123.54,123.25$, $89.09,89.06,81.71,79.07,65.63,48.74,30.55,19.05,18.58,18.54,13.68 . \mathrm{MS}$ (MALDI-TOF): calcd for $\mathrm{C}_{50} \mathrm{H}_{53} \mathrm{~N}_{3} \mathrm{O}_{9} \mathrm{Na}[\mathrm{M}+\mathrm{Na}]^{+} 862.3674$ found 862.4790.

Macrocycle1b. To a $100 \mathrm{~mL}$ two-necked flask charged with $\operatorname{Pd}_{2}(\mathrm{dba})_{3}(22.6 \mathrm{mg}, 0.025$ $\mathrm{mmol})$ and $\mathrm{CuI}(9.4 \mathrm{mg}, 0.049 \mathrm{mmol}), \mathrm{PPh}_{3}(65.6 \mathrm{mg}, 0.25 \mathrm{mmol})$ under argon, $20 \mathrm{~mL}$ degassed $\mathrm{Et}_{3} \mathrm{~N}$ and $40 \mathrm{~mL}$ degassed $\mathrm{DMF}$ were added via syringe. The solution was stirred at $85^{\circ} \mathrm{C}$ for $10 \mathrm{~min}$. A solution of compound $4 \mathbf{b}(52.2 \mathrm{mg}, 0.050 \mathrm{mmol})$ and compound $6 \mathbf{b}(42.0 \mathrm{mg}, 0.050 \mathrm{mmol})$ in $10 \mathrm{~mL}$ DMF was slowly injected into the flask within $48 \mathrm{~h}$ by a syringe pump and then the solution was stirred for further $12 \mathrm{~h}$. The reaction mixture was concentrated in vacuo and purified by column chromatography $\left(\mathrm{CHCl}_{3} / \mathrm{CH}_{3} \mathrm{OH} / \mathrm{AcOH}=30 / 1 / 0.25\right)$ to afford the crude product. The crude product was then washed with a few acetone and methanol to give the desired product $(7.6 \mathrm{mg}, 9 \%)$ as 
a white solid. ${ }^{1} \mathbf{H}$ NMR (400 MHz, DMSO-d 6 ) $\delta 9.04(\mathrm{~d}, J=6.6 \mathrm{~Hz}, 6 \mathrm{H}), 8.14(\mathrm{~s}, 12 \mathrm{H})$, $8.00(\mathrm{~s}, 6 \mathrm{H}), 4.55-4.43(\mathrm{~m}, 6 \mathrm{H}), 4.16-3.98(\mathrm{~m}, 12 \mathrm{H}), 1.57(\mathrm{~m}, 12 \mathrm{H}), 1.42(\mathrm{~m}, 18 \mathrm{H})$, $1.33(\mathrm{~m}, 12 \mathrm{H}), 0.88(\mathrm{~m}, 18 \mathrm{H}) .{ }^{13} \mathbf{C}$ NMR (101 MHz, DMSO-d $) \delta 170.96,162.99,135.05$, $133.49,130.16,130.06,129.41,127.58,121.25,87.59,62.69,47.16,28.68,17.05,15.16$, 12.02.MS (MALDI-TOF): calcd for $\mathrm{C}_{96} \mathrm{H}_{102} \mathrm{~N}_{6} \mathrm{O}_{18} \mathrm{Na}[\mathrm{M}+\mathrm{Na}]^{+} 1649.7143$ found 1650.0584 .

(S)-Octyl-2-(4-fluoro-3,5-diiodobenzamido)propanoate (3c). To a $250 \mathrm{~mL}$ round bottom flask was added 4-fluoro-3,5-diiodobenzoic acid (10.0 g, $25.5 \mathrm{mmol}), 100 \mathrm{~mL}$ redistilled $\mathrm{CH}_{2} \mathrm{Cl}_{2}$ and $2.4 \mathrm{~mL}(\mathrm{COCl})_{2}(\mathrm{Oxalyl}$ chloride) $(3.6 \mathrm{~g}, 28.3 \mathrm{mmol})$, then $0.1 \mathrm{~mL}$ DMF was added dropwise. The solution was stirred for $6 \mathrm{~h}$. After removing the solvent and the redundant $(\mathrm{COCl})_{2}$ under reduced pressure, the residue was resolve in $20 \mathrm{~mL}$ redistilled $\mathrm{CH}_{2} \mathrm{Cl}_{2}$ and added dropwise to a $250 \mathrm{~mL}$ ice-cooled round bottom flask containing $100 \mathrm{~mL}$ redistilled $\mathrm{CH}_{2} \mathrm{Cl}_{2}, 11 \quad \mathrm{~mL}^{2} \mathrm{Et}_{3} \mathrm{~N} \quad(8.0 \mathrm{~g}, 79.5 \mathrm{mmol})$ and $(S)$-octyl-2-aminopropanoate hydrochloride $(6.4 \mathrm{~g}, 26.9 \mathrm{mmol})$. The mixture was stirred for $8 \mathrm{~h}$ at r.t.. The solution was washed with water and brine, and then concentrated in vacuo. The crude product was purified by column chromatography (petroleum ether/acetone $=8 / 1)$ to afford a white solid $(11.5 \mathrm{~g}, 78 \%) .{ }^{1} \mathbf{H}$ NMR $\left(400 \mathrm{MHz}, \mathrm{CDCl}_{3}\right)$ $\delta 8.13(\mathrm{~d}, J=5.4 \mathrm{~Hz}, 2 \mathrm{H}), 6.83(\mathrm{~d}, J=7.2 \mathrm{~Hz}, 1 \mathrm{H}), 4.74(\mathrm{~m}, 1 \mathrm{H}), 4.18(\mathrm{~m}, 2 \mathrm{H}), 1.72-$ $1.60(\mathrm{~m}, 2 \mathrm{H}), 1.51(\mathrm{~d}, J=7.2 \mathrm{~Hz}, 3 \mathrm{H}), 1.40-1.15(\mathrm{~m}, 8 \mathrm{H}), 0.87(\mathrm{t}, J=6.8 \mathrm{~Hz} 3 \mathrm{H}) .{ }^{13} \mathbf{C}$ NMR $\left(101 \mathrm{MHz}, \mathrm{CDCl}_{3}\right) \delta 173.43,173.35,164.47,163.70,163.17,161.24,138.78$ (d, $J$ $=2.2 \mathrm{~Hz}), 133.20(\mathrm{~d}, J=4.1 \mathrm{~Hz}), 129.41,115.91,115.67,80.63(\mathrm{~d}, J=29.8 \mathrm{~Hz}), 66.19$, 48.95, 31.88, 29.27, 28.63, 25.93, 22.76, 18.67, 14.22. HRMS (ESI): calcd for $\mathrm{C}_{18} \mathrm{H}_{24} \mathrm{FI}_{2} \mathrm{NO}_{3} \mathrm{Na}[\mathrm{M}+\mathrm{Na}]^{+} 597.9722$ found 597.9701 .

\section{(S)-Octyl-2-(4-fluoro-3,5-bis(2-(trimethylsilyl)ethynyl)benzamido)propanoate (11c).}

To a $100 \mathrm{~mL}$ two-necked flask charged with 3c (5.7g, $9.9 \mathrm{mmol}), \mathrm{Pd}\left(\mathrm{PPh}_{3}\right)_{2} \mathrm{Cl}_{2}(214.5 \mathrm{mg}$, $0.3 \mathrm{mmol})$ and $\mathrm{CuI}(114.3 \mathrm{mg}, 0.6 \mathrm{mmol})$ under argon, $40 \mathrm{~mL}$ THF and $20 \mathrm{~mL} \mathrm{Et}_{3} \mathrm{~N}$ were added via syringe. The solution was stirred for $10 \mathrm{~min}$ and then 5.6 
mLethynyltrimethylsilane (3.9 g, $40 \mathrm{mmol})$ was injected into the solution. The mixture was stirred overnight at r.t.. After removing the solvent under reduced pressure, the residue was purified by column chromatography (petroleum ether/acetone $=30 / 1)$ to afford a yellow oil (3.6g, 71\%). ${ }^{1} \mathbf{H}$ NMR $\left(400 \mathrm{MHz}, \mathrm{CDCl}_{3}\right) \delta 7.82(\mathrm{~d}, J=6.1 \mathrm{~Hz}, 2 \mathrm{H})$, $6.75(\mathrm{~d}, J=7.2 \mathrm{~Hz}, 1 \mathrm{H}), 4.72(\mathrm{p}, J=7.1 \mathrm{~Hz}, 1 \mathrm{H}), 4.22-4.11(\mathrm{~m}, 2 \mathrm{H}), 1.73-1.60(\mathrm{~m}$, 2H), $1.49(\mathrm{~d}, J=7.1 \mathrm{~Hz}, 3 \mathrm{H}), 1.35-1.19(\mathrm{~m}, 10 \mathrm{H}), 0.86(\mathrm{t}, J=6.8 \mathrm{~Hz}, 3 \mathrm{H}), 0.25(\mathrm{~s}$, 18H). ${ }^{13}$ C NMR (101 MHz, $\left.\mathrm{CDCl}_{3}\right) \delta 173.27,164.97(\mathrm{~d}, J=263.0 \mathrm{~Hz}), 164.43,132.62(\mathrm{~d}$, $J=2.0 \mathrm{~Hz}), 129.94(\mathrm{~d}, J=4.2 \mathrm{~Hz}), 112.79$ (d, $J=16.8 \mathrm{~Hz}), 102.30$ (d, $J=3.6 \mathrm{~Hz}), 96.19$, $66.03,48.82,31.86,29.25,28.61,27.01,25.89,22.74,18.76,14.22$ (d, $J=4.0 \mathrm{~Hz}),-0.19$. HRMS (ESI): calcd for $\mathrm{C}_{28} \mathrm{H}_{42} \mathrm{FNO}_{3} \mathrm{Si}_{2} \mathrm{Na}[\mathrm{M}+\mathrm{Na}]^{+} 538.2579$ found 538.2563.

(S)-Octyl-2-(4-fluoro-3,5-diethynylbenzamido)propanoate (2c). A solution of 11c $(3.0 \mathrm{~g}, 5.8 \mathrm{mmol})$ in $30 \mathrm{~mL}$ THF was added a solution of tetrabutylammonium fluoride $(4.5 \mathrm{~g}, 17.2 \mathrm{mmol})$ in $10 \mathrm{~mL}$ THF at r.t.. The mixture was stirred for 30min. After removing the solvent under reduced pressure, the residue was re-dissolved in $30 \mathrm{~mL}$ $\mathrm{CHCl}_{3}$ and washed with water and brine. Then the organic layer was dried over anhydrous $\mathrm{Na}_{2} \mathrm{SO}_{4}$ and the solvent was removed under reduced pressure. The crude product was purified by column chromatography (petroleum ether/acetone $=10 / 1$ ) to afford a white solid (2.0g, 93\%). ${ }^{1} \mathbf{H}$ NMR (400 MHz, $\left.\mathrm{CDCl}_{3}\right) \delta 7.90(\mathrm{~d}, J=6.1 \mathrm{~Hz}, 2 \mathrm{H})$, $6.76(\mathrm{~s}, 1 \mathrm{H}), 4.74(\mathrm{p}, J=7.1 \mathrm{~Hz}, 1 \mathrm{H}), 4.23-4.12(\mathrm{~m}, 2 \mathrm{H}), 3.37$ (s, 2H), $1.69-1.61$ (m, 2H), $1.51(\mathrm{~d}, J=7.1 \mathrm{~Hz}, 3 \mathrm{H}), 1.37-1.20(\mathrm{~m}, 10 \mathrm{H}), 0.87(\mathrm{t}, J=6.8 \mathrm{~Hz}, 3 \mathrm{H}) .{ }^{13} \mathbf{C}$ NMR $\left(101 \mathrm{MHz} \mathrm{CDCl}_{3}\right) \delta 173.24,166.86,164.23,133.35$ (d, $\left.J=2.1 \mathrm{~Hz}\right), 130.32$ (d, $J=4.3$ Hz), 111.89 (d, $J=16.3 \mathrm{~Hz}), 84.35$ (d, $J=3.7 \mathrm{~Hz}), 75.44,66.13,48.90,31.88,29.28$, 28.64, 25.93, 22.76, 18.76, 14.21. HRMS (ESI): calcd for $\mathrm{C}_{22} \mathrm{H}_{26} \mathrm{FNO}_{3} \mathrm{Na}[\mathrm{M}+\mathrm{Na}]^{+}$ 394.1789 found 394.1778 .

Compound 4c. To a $100 \mathrm{~mL}$ two-necked flask charged with 3c (575.2 mg, $1.0 \mathrm{mmol}), 2 \mathrm{c}$ (185.7 mg, $0.50 \mathrm{mmol}), \mathrm{Pd}_{2}(\mathrm{dba})_{3}(13.7 \mathrm{mg}, 0.015 \mathrm{mmol})$ and $\mathrm{CuI}(5.7 \mathrm{mg}, 0.030 \mathrm{mmol})$, $\mathrm{PPh}_{3}\left(39.8 \mathrm{mg}, 0.15 \mathrm{mmol}\right.$ ) under argon, $50 \mathrm{~mL}$ degassed $\mathrm{Et}_{3} \mathrm{~N}$ was added via syringe. 
The mixture was stirred at $75^{\circ} \mathrm{C}$ for $12 \mathrm{~h}$, and then removed the solvent under reduced pressure. The residue was purified by column chromatography $\left(\mathrm{CHCl}_{3} / \mathrm{THF}=40 / 1\right)$ to afford a light yellow solid (239.2 mg, 38). ${ }^{1} \mathbf{H}$ NMR (400 MHz, $\left.\mathrm{CDCl}_{3}\right) \delta 8.23(\mathrm{dd}, J=5.5$, $2.2 \mathrm{~Hz}, 2 \mathrm{H}), 8.00(\mathrm{~d}, J=6.1 \mathrm{~Hz}, 2 \mathrm{H}), 7.94(\mathrm{dd}, J=6.0,2.2 \mathrm{~Hz}, 2 \mathrm{H}), 6.93(\mathrm{~d}, J=7.3 \mathrm{~Hz}$, 1H), $6.86(\mathrm{~d}, J=7.3 \mathrm{~Hz}, 2 \mathrm{H}), 4.85-4.73(\mathrm{~m}, 3 \mathrm{H}), 4.31-4.11(\mathrm{~m}, 6 \mathrm{H}), 1.76-1.63(\mathrm{~m}$, $6 \mathrm{H}), 1.57-1.51(\mathrm{~m}, 9 \mathrm{H}), 1.40-1.22(\mathrm{~m}, 30 \mathrm{H}), 0.88(\mathrm{t}, J=6.8 \mathrm{~Hz}, 9 \mathrm{H}) .{ }^{13} \mathbf{C}$ NMR $(101$ $\left.\mathrm{MHz}, \mathrm{CDCl}_{3}\right) \delta 173.47,173.39,164.25,163.78,162.93,162.29,139.48,133.15,132.50$, 131.66, 112.07, 87.79, $81.84(\mathrm{~d}, J=26.4 \mathrm{~Hz}), 77.42(\mathrm{~d}, J=11.5 \mathrm{~Hz}), 77.16,76.84,66.15$, 48.97, 31.90, 29.30, 28.66, 25.95, 22.77, 18.63, 14.23. MS (MALDI-TOF): calcd for $\mathrm{C}_{58} \mathrm{H}_{72} \mathrm{~F}_{3} \mathrm{I}_{2} \mathrm{~N}_{3} \mathrm{O}_{9} \mathrm{Na}[\mathrm{M}+\mathrm{Na}]^{+} 1288.3202$ found 1288.6489 .

Compound 5c. To a $50 \mathrm{~mL}$ two-necked flask charged with compound 4c (253.2 mg, 0.20 $\mathrm{mmol}), \mathrm{Pd}\left(\mathrm{PPh}_{3}\right)_{2} \mathrm{Cl}_{2}(4.3 \mathrm{mg}, 0.006 \mathrm{mmol})$ and $\mathrm{CuI}(2.3 \mathrm{mg}, 0.012 \mathrm{mmol})$ under argon, $15 \mathrm{~mL} \mathrm{THF}$ and $8 \mathrm{~mL} \mathrm{Et}_{3} \mathrm{~N}$ were added via syringe. The solution was stirred for $10 \mathrm{~min}$ and then $0.11 \mathrm{~mL}$ ethynyltrimethylsilane $(78.4 \mathrm{mg}, 0.8 \mathrm{mmol})$ was injected into the solution. The mixture was stirred overnight at room temperature After removing the solvent under reduced pressure, the residue was purified by column chromatography $\left(\mathrm{CHCl}_{3} / \mathrm{THF}=60 / 1\right)$ to afford a yellow oil $(217.8 \mathrm{mg}, 90 \%) .{ }^{1} \mathbf{H} \mathbf{N M R}\left(400 \mathrm{MHz}, \mathrm{CDCl}_{3}\right)$ $\delta 8.00(\mathrm{~d}, J=6.0 \mathrm{~Hz}, 2 \mathrm{H}), 7.95(\mathrm{dd}, J=6.1,2.3 \mathrm{~Hz}, 2 \mathrm{H}), 7.91(\mathrm{dd}, J=6.1,2.3 \mathrm{~Hz}, 2 \mathrm{H})$, $6.88(\mathrm{~d}, J=7.3 \mathrm{~Hz}, 1 \mathrm{H}), 6.81(\mathrm{~d}, J=7.3 \mathrm{~Hz}, 2 \mathrm{H}), 4.85-4,72(\mathrm{~m}, 3 \mathrm{H}), 4.26-4.12(\mathrm{~m}$, $6 \mathrm{H}), 1.73-1.63(\mathrm{~m}, 6 \mathrm{H}), 1.57-1.51(\mathrm{~m}, 9 \mathrm{H}), 1.38-1.21(\mathrm{~m}, 30 \mathrm{H}), 0.87(\mathrm{t}, J=6.7 \mathrm{~Hz}$, 9H), 0.28 (s, 18H). ${ }^{13}$ C NMR (101 MHz, $\left.\mathrm{CDCl}_{3}\right)$ 8173.27, 166.03, 164.38, 164.29, 163.40, 133.38, 132.99, 132.34, 130.27, 113.13 (d, $J=16.5 \mathrm{~Hz}), 112.27,102.85,96.03$, $87.98,87.48,66.11,48.93,31.89,29.29,28.66,25.94,22.77,18.79,14.22,-0.15 . \mathrm{MS}$ (MALDI-TOF): calcd for $\mathrm{C}_{68} \mathrm{H}_{90} \mathrm{~F}_{3} \mathrm{~N}_{3} \mathrm{O}_{9} \mathrm{Si}_{2} \mathrm{Na}[\mathrm{M}+\mathrm{Na}]^{+} 1228.6060$ found 1228.9036 .

Compound 6c. A solution of compound $\mathbf{5 c}(181.0 \mathrm{mg}, 0.15 \mathrm{mmol})$ in $10 \mathrm{~mL}$ THF was added a solution of tetrabutylammonium fluoride $(117.5 \mathrm{mg}, 0.45 \mathrm{mmol})$ in $3 \mathrm{~mL}$ THF at r.t.. The mixture was stirred for 30min. After removing the solvent under reduced 
pressure, the residue was re-dissolved in $15 \mathrm{~mL} \mathrm{CHCl}_{3}$ and washed with water and brine. Then the organic layer was dried over anhydrous $\mathrm{Na}_{2} \mathrm{SO}_{4}$ and the solvent was removed under reduced pressure. The crude product was purified by column chromatography $\left(\mathrm{CHCl}_{3} / \mathrm{THF}=30 / 1\right)$ to afford a light yellow solid $(146.5 \mathrm{mg}, 92 \%) .{ }^{1} \mathbf{H}$ NMR $(400 \mathrm{MHz}$, $\left.\mathrm{CDCl}_{3}\right) \delta 7.94(\mathrm{~d}, J=2.3 \mathrm{~Hz}, 2 \mathrm{H}), 7.89$ (t, $\left.J=1.6 \mathrm{~Hz}, 2 \mathrm{H}\right), 7.78$ (q, $\left.J=1.4 \mathrm{~Hz}, 2 \mathrm{H}\right), 7.00$ - $6.83(\mathrm{~m}, 3 \mathrm{H}), 4.87-4.75(\mathrm{~m}, 3 \mathrm{H}), 4.25-4.13(\mathrm{~m}, 6 \mathrm{H}), 3.17$ (s, 2H), $1.75-1.62(\mathrm{~m}$, $6 \mathrm{H}), 1.55(\mathrm{~d}, J=7.1 \mathrm{~Hz}, 9 \mathrm{H}), 1.39-1.23(\mathrm{~m}, 30 \mathrm{H}), 0.88(\mathrm{t}, J=6.8 \mathrm{~Hz}, 9 \mathrm{H}) .{ }^{13} \mathbf{C}$ NMR $\left(101 \mathrm{MHz}, \mathrm{CDCl}_{3}\right) \delta 173.41,173.33,164.31,163.62,133.79,133.15,132.76,130.36$, $112.61-111.84,87.67(\mathrm{~d}, J=7.5 \mathrm{~Hz}), 84.45,75.45,66.11,48.95,31.89,29.29,28.65$, 25.94, 22.76, 18.66, 14.22.MS (MALDI-TOF): calcd for $\mathrm{C}_{62} \mathrm{H}_{74} \mathrm{~F}_{3} \mathrm{~N}_{3} \mathrm{O}_{9} \mathrm{Na}[\mathrm{M}+\mathrm{Na}]^{+}$ 1084.5269 found 1084.7414.

Macrocycle1c. To a $100 \mathrm{~mL}$ two-necked flask charged with $\operatorname{Pd}_{2}(\mathrm{dba})_{3}(22.6 \mathrm{mg}, 0.025$ $\mathrm{mmol})$ and $\mathrm{CuI}(9.4 \mathrm{mg}, 0.049 \mathrm{mmol}), \mathrm{PPh}_{3}(65.6 \mathrm{mg}, 0.25 \mathrm{mmol})$ under argon, $20 \mathrm{~mL}$ degassed $\mathrm{Et}_{3} \mathrm{~N}$ and $40 \mathrm{~mL}$ degassed $\mathrm{DMF}$ were added via syringe. The solution was stirred at $85^{\circ} \mathrm{C}$ for $10 \mathrm{~min}$. A solution of compound $4 \mathbf{c}(63.3 \mathrm{mg}, 0.050 \mathrm{mmol})$ and compound 6c $(53.1 \mathrm{mg}, 0.050 \mathrm{mmol})$ in $10 \mathrm{~mL}$ DMF was slowly injected into the flask within $48 \mathrm{~h}$ by a syringe pump and then the solution was stirred for further $12 \mathrm{~h}$. The reaction mixture was concentrated in vacuo and purified by column chromatography $\left(\mathrm{CHCl}_{3} / \mathrm{THF}=10 / 1\right.$ and then THF $)$ to afford the crude product. The crude product was then washed with a few methanol to give the desired product $(18.6 \mathrm{mg}, 18 \%)$ as a yellow solid. ${ }^{1} \mathbf{H}$ NMR $\left(400 \mathrm{MHz}, \mathrm{DMSO}-d_{6}\right) \delta 8.85-8.76(\mathrm{~m}, 6 \mathrm{H}), 8.35-7.85(\mathrm{~m}, 12 \mathrm{H}), 4.55$ $-4.41(\mathrm{~m}, 6 \mathrm{H}), 4.20-3.96(\mathrm{~m}, 12 \mathrm{H}), 1.60$ (p, $J=6.8 \mathrm{~Hz}, 12 \mathrm{H}), 1.46(\mathrm{~d}, J=7.2 \mathrm{~Hz}, 18 \mathrm{H})$, $1.34-1.11(\mathrm{~m}, 60 \mathrm{H}), 0.82(\mathrm{t}, J=6.6 \mathrm{~Hz}, 18 \mathrm{H}) .{ }^{13} \mathbf{C}$ NMR $\left(101 \mathrm{MHz}, \mathrm{DMSO}-d_{6}\right) \delta$ $172.19,164.19,163.32,161.57,133.04129 .81,110.70(\mathrm{~d}, J=16.0 \mathrm{~Hz}), 87.08,64.29$, 48.56, 31.10, 28.54, 28.04, 25.24, 21.97, 16.43, 13.68. MS (MALDI-TOF): calcd for $\mathrm{C}_{120} \mathrm{H}_{144} \mathrm{~F}_{6} \mathrm{~N}_{6} \mathrm{O}_{18} \mathrm{Na}[\mathrm{M}+\mathrm{Na}]^{+} 2094.0334$ found 2094.2743 .

(S)-Butyl-2-(4-fluoro-3,5-diiodobenzamido)propanoate (3d). To a $250 \mathrm{~mL}$ round 
bottom flask was added 4-fluoro-3,5-diiodobenzoic acid (10.0 g, $25.5 \mathrm{mmol}), 100 \mathrm{~mL}$ redistilled $\mathrm{CH}_{2} \mathrm{Cl}_{2}$ and $2.4 \mathrm{~mL}(\mathrm{COCl})_{2}$ (oxalyl chloride) $(3.6 \mathrm{~g}, 28.3 \mathrm{mmol})$, then $0.1 \mathrm{~mL}$ DMF was added dropwise. The solution was stirred for $6 \mathrm{~h}$. After removing the solvent and the redundant $(\mathrm{COCl})_{2}$ under reduced pressure, the residue was resolve in $20 \mathrm{~mL}$ redistilled $\mathrm{CH}_{2} \mathrm{Cl}_{2}$ and added dropwise to a $250 \mathrm{~mL}$ ice-cooled round bottom flask containing $100 \mathrm{~mL}$ redistilled $\mathrm{CH}_{2} \mathrm{Cl}_{2}, 11 \mathrm{~mL} \mathrm{Et}_{3} \mathrm{~N}(8.0 \mathrm{~g}, 79.5 \mathrm{mmol})$ and (S)-butyl-2-aminopropanoate hydrochloride (4.9 g, $27.0 \mathrm{mmol})$. The mixture was stirred for $8 \mathrm{~h}$ at r.t.. The solution was washed with water and brine, and then concentrated in vacuo. The crude product was purified by column chromatography (petroleum ether/acetone $=5 / 1)$ to afford a white solid $(10.8 \mathrm{~g}, 82 \%) .{ }^{1} \mathbf{H}$ NMR $\left(400 \mathrm{MHz}, \mathrm{CDCl}_{3}\right) \delta$ $8.15(\mathrm{~d}, J=5.4 \mathrm{~Hz}, 2 \mathrm{H}), 6.72(\mathrm{~d}, J=7.2 \mathrm{~Hz}, 1 \mathrm{H}), 4.74(\mathrm{t}, J=7.2 \mathrm{~Hz}, 1 \mathrm{H}), 4.21(\mathrm{td}, J=$ 6.6, 2.5 Hz, 2H), $1.72-1.63(\mathrm{~m}, 2 \mathrm{H}), 1.52(\mathrm{~d}, J=7.1 \mathrm{~Hz}, 3 \mathrm{H}), 1.47-1.34(\mathrm{~m}, 2 \mathrm{H}), 0.96$ $(\mathrm{t}, J=7.4 \mathrm{~Hz}, 3 \mathrm{H}) .{ }^{13} \mathbf{C} \mathbf{N M R}\left(101 \mathrm{MHz}, \mathrm{CDCl}_{3}\right) \delta 173.13,163.01,162.36(\mathrm{~d}, J=247.7$ H), 138.62 (d, $J=2.2 \mathrm{~Hz}), 133.13$ (d, $J=4.1 \mathrm{~Hz}), 80.53$ (d, $J=29.8 \mathrm{~Hz}), 65.75,48.82$, 30.52, 19.04, 18.59, 13.67. HRMS (ESI): calcd for $\mathrm{C}_{14} \mathrm{H}_{16} \mathrm{FI}_{2} \mathrm{NO}_{3} \mathrm{Na}[\mathrm{M}+\mathrm{Na}]^{+} 541.9096$ found 541.9101 .

\section{(S)-Butyl-2-(4-fluoro-3,5-bis(2-(trimethylsilyl)ethynyl)benzamido)propanoate(11d).}

To a $100 \mathrm{~mL}$ two-necked flask charged with 3d (5.2g, $10.0 \mathrm{mmol}), \mathrm{Pd}\left(\mathrm{PPh}_{3}\right)_{2} \mathrm{Cl}_{2}(214.5$ $\mathrm{mg}, 0.3 \mathrm{mmol})$ and $\mathrm{CuI}(114.3 \mathrm{mg}, 0.6 \mathrm{mmol})$ under argon, $40 \mathrm{~mL}$ THF and $20 \mathrm{~mL} \mathrm{Et}_{3} \mathrm{~N}$ were added via syringe. The solution was stirred for $10 \mathrm{~min}$ and then 5.6

mLethynyltrimethylsilane $(3.9 \mathrm{~g}, 40 \mathrm{mmol})$ was injected into the solution. The mixture was stirred overnight at r.t.. After removing the solvent under reduced pressure, the residue was purified by column chromatography (petroleum ether/acetone $=25 / 1$ ) to afford a yellow oil (3.7g, 80\%). ${ }^{1} \mathbf{H}$ NMR $\left(400 \mathrm{MHz}, \mathrm{CDCl}_{3}\right) \delta 7.83(\mathrm{~d}, J=6.1 \mathrm{~Hz}, 2 \mathrm{H})$, $6.72(\mathrm{~d}, J=7.2 \mathrm{~Hz}, 1 \mathrm{H}), 4.74(\mathrm{p}, J=7.1 \mathrm{~Hz}, 1 \mathrm{H}), 4.19(\mathrm{td}, J=6.6,3.0 \mathrm{~Hz}, 2 \mathrm{H}), 1.71-$ $1.61(\mathrm{~m}, 2 \mathrm{H}), 1.50(\mathrm{~d}, J=7.2 \mathrm{~Hz}, 3 \mathrm{H}), 1.45-1.34(\mathrm{~m}, 2 \mathrm{H}), 0.95$ (t, $J=7.4 \mathrm{~Hz}, 3 \mathrm{H}), 0.26$

(s, 18H). ${ }^{13}$ C NMR (101 MHz, $\left.\mathrm{CDCl}_{3}\right) \delta 173.46,166.47,164.60,132.79,130.11,113.00$ 
(d, $J=16.8 \mathrm{~Hz}), 102.53$ (d, $J=3.7 \mathrm{~Hz}), 96.37,65.96,49.00,30.82,19.33,19.01,13.96$, -0.00.HRMS (ESI): calcd for $\mathrm{C}_{24} \mathrm{H}_{34} \mathrm{FNO}_{3} \mathrm{Si}_{2} \mathrm{Na}[\mathrm{M}+\mathrm{Na}]^{+} 482.1953$ found 482.1958 .

(S)-Butyl-2-(4-fluoro-3,5-diethynylbenzamido)propanoate (2d). A solution of 11d $(2.7 \mathrm{~g}, 5.9 \mathrm{mmol})$ in $30 \mathrm{~mL}$ THF was added a solution of tetrabutylammonium fluoride (4.6g, $17.7 \mathrm{mmol}$ ) in $10 \mathrm{~mL}$ THF at r.t. The mixture was stirred for $30 \mathrm{~min}$. After removing the solvent under reduced pressure, the residue was redissolvedin $30 \mathrm{~mL}$ $\mathrm{CHCl}_{3}$ and washed with water and brine. Then the organic layer was dried over anhydrous $\mathrm{Na}_{2} \mathrm{SO}_{4}$ and the solvent was removed under reduced pressure. The crude product was purified by column chromatography (petroleum ether/acetone $=8 / 1$ ) to afford a white solid (1.6g, 86\%). ${ }^{1} \mathbf{H}$ NMR (400 MHz, $\left.\mathrm{CDCl}_{3}\right) \delta 7.91(\mathrm{~d}, J=6.1 \mathrm{~Hz}, 2 \mathrm{H})$, $6.78(\mathrm{~d}, J=7.3 \mathrm{~Hz}, 1 \mathrm{H}), 4.74(\mathrm{p}, J=7.1 \mathrm{~Hz}, 1 \mathrm{H}), 4.20(\mathrm{td}, J=6.7,2.8 \mathrm{~Hz}, 2 \mathrm{H}), 3.38$ (s, 2H), $1.71-1.60(\mathrm{~m}, 2 \mathrm{H}), 1.52(\mathrm{~d}, J=7.1 \mathrm{~Hz}, 3 \mathrm{H}), 1.47-1.33(\mathrm{~m}, 2 \mathrm{H}), 0.95(\mathrm{t}, J=7.4$ $\mathrm{Hz}, 3 \mathrm{H}){ }^{13} \mathbf{C}$ NMR $\left(101 \mathrm{MHz}, \mathrm{CDCl}_{3}\right) \delta 173.25,166.86,164.23,133.35$ (d, $\left.J=2.2 \mathrm{~Hz}\right)$, 130.31 (d, $J=4.3 \mathrm{~Hz}), 111.90$ (d, $J=16.4 \mathrm{~Hz}), 84.37$ (d, $J=3.6 \mathrm{~Hz}), 75.44,65.84,48.90$, 30.66, 19.17, 18.78, 13.80. HRMS (ESI): calcd for $\mathrm{C}_{18} \mathrm{H}_{18} \mathrm{FNO}_{3} \mathrm{Na}[\mathrm{M}+\mathrm{Na}]+338.1163$ found 338.1163 .

Compound 4d. To a $100 \mathrm{~mL}$ two-necked flask charged with $3 d$ (519.0 mg, $1.0 \mathrm{mmol})$, 2d (157.7 mg, $0.50 \mathrm{mmol}), \mathrm{Pd}_{2}(\mathrm{dba})_{3}(13.7 \mathrm{mg}, 0.015 \mathrm{mmol})$ and $\mathrm{CuI}(5.7 \mathrm{mg}, 0.030$ $\mathrm{mmol}), \mathrm{PPh}_{3}\left(39.8 \mathrm{mg}, 0.15 \mathrm{mmol}\right.$ ) under argon, $50 \mathrm{~mL}$ degassed $\mathrm{Et}_{3} \mathrm{~N}$ was added via syringe. The mixture was stirred at $75^{\circ} \mathrm{C}$ for $12 \mathrm{~h}$, and then removed the solvent under reduced pressure. The residue was purified by column chromatography $\left(\mathrm{CHCl}_{3} / \mathrm{THF}=\right.$ 40/1) to afford a light yellow solid (243.8 mg, 44\%). ${ }^{1} \mathbf{H}$ NMR (400 MHz, $\left.\mathrm{CDCl}_{3}\right) \delta 8.23$ (dd, $J=5.5,2.2 \mathrm{~Hz}, 2 \mathrm{H}), 7.99(\mathrm{~d}, J=6.0 \mathrm{~Hz}, 2 \mathrm{H}), 7.94(\mathrm{dd}, J=6.0,2.2 \mathrm{~Hz}, 2 \mathrm{H}), 7.04-$ $6.96(\mathrm{~m}, 1 \mathrm{H}), 6.91(\mathrm{~d}, J=7.4 \mathrm{~Hz}, 2 \mathrm{H}), 4.88-4.70(\mathrm{~m}, 3 \mathrm{H}), 4.30-4.12(\mathrm{~m}, 6 \mathrm{H}), 1.67$ (p, $J=6.9 \mathrm{~Hz}, 6 \mathrm{H}), 1.61-1.52(\mathrm{~m}, 10 \mathrm{H}), 1.47-1.35(\mathrm{~m}, 8 \mathrm{H}), 0.95(\mathrm{t}, J=7.4 \mathrm{~Hz}, 6 \mathrm{H}) .{ }^{13} \mathbf{C}$ NMR (101 MHz, $\left.\mathrm{CDCl}_{3}\right) \delta 173.33,163.79,162.32,139.44,133.12,132.46,131.74$, 111.65, 87.81, 65.86, 48.98, 30.69, 19.20, 18.70, 13.82, 0.14. MS (MALDI-TOF): calcd 
for $\mathrm{C}_{46} \mathrm{H}_{48} \mathrm{~F}_{3} \mathrm{I}_{2} \mathrm{~N}_{3} \mathrm{O}_{9} \mathrm{Na}[\mathrm{M}+\mathrm{Na}]^{+} 1120.1324$ found 1120.3549 .

Compound 5d. To a $50 \mathrm{~mL}$ two-necked flask charged with compound $4 \mathbf{d}$ (219.5 mg, $0.20 \mathrm{mmol}), \mathrm{Pd}\left(\mathrm{PPh}_{3}\right)_{2} \mathrm{Cl}_{2}(4.3 \mathrm{mg}, 0.006 \mathrm{mmol})$ and $\mathrm{CuI}(2.3 \mathrm{mg}, 0.012 \mathrm{mmol})$ under argon, $15 \mathrm{~mL}$ THF and $8 \mathrm{~mL} \mathrm{Et}_{3} \mathrm{~N}$ were added via syringe. The solution was stirred for $10 \mathrm{~min}$ and then $0.11 \mathrm{mLethynyltrimethylsilane}(78.4 \mathrm{mg}, 0.8 \mathrm{mmol})$ was injected into the solution. The mixture was stirred overnight at r.t.. After removing the solvent under reduced pressure, the residue was purified by column chromatography $\left(\mathrm{CHCl}_{3} / \mathrm{THF}=\right.$ 60/1) to afford a yellow oil (191.1 mg, 92\%). ${ }^{1} \mathbf{H}$ NMR (400 MHz, $\left.\mathrm{CDCl}_{3}\right) \delta 8.02(\mathrm{~d}, J=$ $6.0 \mathrm{~Hz}, 2 \mathrm{H}), 7.93(\mathrm{ddd}, J=12.6,6.1,2.3 \mathrm{~Hz}, 4 \mathrm{H}), 7.10$ (d, $J=7.4 \mathrm{~Hz}, 1 \mathrm{H}), 6.96$ (d, $J=$ $7.3 \mathrm{~Hz}, 2 \mathrm{H}), 4.88-4.74(\mathrm{~m}, 3 \mathrm{H}), 4.26-4.11(\mathrm{~m}, 6 \mathrm{H}), 1.73-1.61(\mathrm{~m}, 6 \mathrm{H}), 1.55(\mathrm{t}, J=$ $7.0 \mathrm{~Hz}, 9 \mathrm{H}), 1.48-1.34(\mathrm{~m}, 6 \mathrm{H}), 0.95(\mathrm{t}, J=7.4 \mathrm{~Hz}, 9 \mathrm{H}), 0.28(\mathrm{~s}, 18 \mathrm{H}) .{ }^{13} \mathbf{C}$ NMR $(101$ $\left.\mathrm{MHz}, \mathrm{CDCl}_{3}\right) \delta 173.32,165.97,164.46,164.36,163.35,133.54,133.08,132.33,130.51$ $130.26(\mathrm{~d}, J=3.8 \mathrm{~Hz}), 113.11(\mathrm{~d}, J=16.4 \mathrm{~Hz}), 112.28(\mathrm{~d}, J=16.0 \mathrm{~Hz}), 111.94(\mathrm{~d}, J=$ 15.9 Hz), 102.79, 96.05, 87.93, 87.45, 65.76, 48.95 (d, $J=3.0 \mathrm{~Hz}), 30.68,19.17,18.65$, 13.77, -0.17. MS (MALDI-TOF): calcd for $\mathrm{C}_{56} \mathrm{H}_{66} \mathrm{~F}_{3} \mathrm{~N}_{3} \mathrm{O}_{9} \mathrm{Si}_{2} \mathrm{Na}[\mathrm{M}+\mathrm{Na}]^{+} 1060.4182$ found 1060.5063 .

Compound 6d. A solution of compound $\mathbf{5 d}(155.7 \mathrm{mg}, 0.15 \mathrm{mmol})$ in $10 \mathrm{~mL}$ THF was added a solution of tetrabutylammonium fluoride $(117.5 \mathrm{mg}, 0.45 \mathrm{mmol})$ in $3 \mathrm{~mL}$ THF at r.t.. The mixture was stirred for 30min. After removing the solvent under reduced pressure, the residue was redissolved in $15 \mathrm{~mL} \mathrm{CHCl}_{3}$ and washed with water and brine. Then the organic layer was dried over anhydrous $\mathrm{Na}_{2} \mathrm{SO}_{4}$ and the solvent was removed under reduced pressure. The crude product was purified by column chromatography $\left(\mathrm{CHCl}_{3} / \mathrm{THF}=25 / 1\right)$ to afford a light yellow solid $(122.7 \mathrm{mg}, 92 \%) .{ }^{\mathbf{1}} \mathbf{H} \mathbf{~ N M R}(400 \mathrm{MHz}$, $\left.\mathrm{CDCl}_{3}\right) \delta 8.15-7.85(\mathrm{~m}, 6 \mathrm{H}), 6.87(\mathrm{~d}, J=7.2 \mathrm{~Hz}, 1 \mathrm{H}), 6.81(\mathrm{~d}, J=7.3 \mathrm{~Hz}, 2 \mathrm{H}), 4.91-$ $4.66(\mathrm{~m}, 3 \mathrm{H}), 4.35-4.10(\mathrm{~m}, 6 \mathrm{H}), 3.40(\mathrm{~s}, 2 \mathrm{H}), 1.75-1.62(\mathrm{~m}, 6 \mathrm{H}), 1.61-1.48(\mathrm{~m}$, 10H), $1.47-1.33(\mathrm{~m}, 6 \mathrm{H}), 0.95(\mathrm{t}, J=7.4 \mathrm{~Hz}, 9 \mathrm{H}) .{ }^{13} \mathbf{C}$ NMR (101 MHz, DMSO-d 6 ) $\delta$ $172.38,164.91,163.43,163.39,162.32,133.90(\mathrm{~d}, J=39.2 \mathrm{~Hz}), 133.21,130.75,130.54$ 
(d, $J=3.9 \mathrm{~Hz}), 110.98(\mathrm{~d}, J=15.7 \mathrm{~Hz}), 110.71(\mathrm{~d}, J=15.8 \mathrm{~Hz}), 110.50(\mathrm{~d}, J=16.0 \mathrm{~Hz})$, $88.05,87.37,87.07,75.18,64.17,48.66,30.14,18.52,16.60,13.48$. MS (MALDI-TOF): calcd for $\mathrm{C}_{50} \mathrm{H}_{50} \mathrm{~F}_{3} \mathrm{~N}_{3} \mathrm{O}_{9} \mathrm{Na}[\mathrm{M}+\mathrm{Na}]^{+} 916.3391$ found 916.5231 .

Macrocycle1d. To a $100 \mathrm{~mL}$ two-necked flask charged with $\operatorname{Pd}_{2}(\mathrm{dba})_{3}(22.6 \mathrm{mg}, 0.025$ $\mathrm{mmol}$ ) and $\mathrm{CuI}(9.4 \mathrm{mg}, 0.049 \mathrm{mmol}), \mathrm{PPh}_{3}(65.6 \mathrm{mg}, 0.25 \mathrm{mmol})$ under argon, $20 \mathrm{~mL}$ degassed $\mathrm{Et}_{3} \mathrm{~N}$ and $40 \mathrm{~mL}$ degassed $\mathrm{DMF}$ were added via syringe. The solution was stirred at $85^{\circ} \mathrm{C}$ for $10 \mathrm{~min}$. A solution of compound $4 \mathbf{d}(54.9 \mathrm{mg}, 0.050 \mathrm{mmol})$ and compound 6d (44.7 mg, $0.050 \mathrm{mmol})$ in $10 \mathrm{~mL}$ DMF was slowly injected into the flask within $48 \mathrm{~h}$ by a syringe pump and then the solution was stirred for further $12 \mathrm{~h}$. The reaction mixture was concentrated in vacuo and purified by column chromatography $\left(\mathrm{CHCl}_{3} / \mathrm{THF}=10 / 1\right.$ and then THF $)$ to afford the crude product. The crude product was then washed with a few acetone and methanol to give the desired product $(9.2 \mathrm{mg}, 11 \%)$ as a yellow solid. ${ }^{1} \mathbf{H}$ NMR $\left(400 \mathrm{MHz}, \mathrm{DMSO}-d_{6}\right) \delta 8.89(\mathrm{~d}, J=6.3 \mathrm{~Hz}, 6 \mathrm{H}), 8.03(\mathrm{~d}, J=$ $6.3 \mathrm{~Hz}, 12 \mathrm{H}), 4.54-4.35(\mathrm{~m}, 6 \mathrm{H}), 4.25-3.92(\mathrm{~m}, 12 \mathrm{H}), 1.63-1.53(\mathrm{~m}, 10 \mathrm{H}), 1.50-$ $1.42(\mathrm{~m}, 14 \mathrm{H}), 1.40-1.30(\mathrm{~m}, 13 \mathrm{H}), 1.26-1.21(\mathrm{~m}, 5 \mathrm{H}), 0.88(\mathrm{t}, J=7.4 \mathrm{~Hz}, 18 \mathrm{H}) .{ }^{13} \mathbf{C}$ NMR (101 MHz, DMSO- $\left.d_{6}\right) \delta 172.37,163.21,162.25,161.53,133.18,129.97,110.70$ (d, $J=15.5 \mathrm{~Hz}), 87.19,64.08,48.74,35.72,30.72,30.12,18.46,16.50,13.42 . \mathrm{MS}$ (MALDI-TOF): calcd for $\mathrm{C}_{96} \mathrm{H}_{96} \mathrm{~F}_{6} \mathrm{~N}_{6} \mathrm{O}_{18} \mathrm{Na}[\mathrm{M}+\mathrm{Na}]^{+} 1757.6578$ found 1757.9289. (S)-Octyl-2-(4-methyl-3,5-diiodobenzamido)propanoate (3f). To a $250 \mathrm{~mL}$ round bottom flask was added 3,5-diiodo-4-methylbenzoic acid (10.0 g, $25.8 \mathrm{mmol}), 100 \mathrm{~mL}$ redistilled $\mathrm{CH}_{2} \mathrm{Cl}_{2}$ and $2.4 \mathrm{~mL}(\mathrm{COCl})_{2}$ (Oxalyl chloride) $(3.6 \mathrm{~g}, 28.4 \mathrm{mmol})$, then $0.1 \mathrm{~mL}$ DMF was added dropwise. The solution was stirred for $6 \mathrm{~h}$. After removing the solvent and the redundant $(\mathrm{COCl})_{2}$ under reduced pressure, the residue was resolve in $20 \mathrm{~mL}$ redistilled $\mathrm{CH}_{2} \mathrm{Cl}_{2}$ and added dropwise to a $250 \mathrm{~mL}$ ice-cooled round bottom flask containing $100 \mathrm{~mL}$ redistilled $\mathrm{CH}_{2} \mathrm{Cl}_{2}, 11 \mathrm{~mL} \mathrm{Et}_{3} \mathrm{~N}(8.0 \mathrm{~g}, 79.5 \mathrm{mmol})$ and (S)-octyl-2-aminopropanoate hydrochloride $(6.4 \mathrm{~g}, 26.9 \mathrm{mmol})$. The mixture was stirred for $8 \mathrm{~h}$ at r.t.. The solution was washed with water and brine, and then concentrated in 
vacuo. The crude product was purified by column chromatography (petroleum ether/acetone $=8 / 1)$ to afford a white solid $(11.8 \mathrm{~g}, 80 \%) .{ }^{1} \mathbf{H} \mathbf{~ N M R}\left(400 \mathrm{MHz}, \mathrm{CDCl}_{3}\right) \delta$ 8.19 (s, 2H), 6.99 (d, J= $7.4 \mathrm{~Hz}, 1 \mathrm{H}), 4.76$ (p, J=7.3 Hz, 1H), $4.25-4.12$ (m, 2H), 2.76 (s, 3H), $1.74-1.60(\mathrm{~m}, 2 \mathrm{H}), 1.51$ (d, $J=7.1 \mathrm{~Hz}, 3 \mathrm{H}), 1.41-1.16(\mathrm{~m}, 10 \mathrm{H}), 0.93-0.83$ (m, 3H). ${ }^{13} \mathbf{C}$ NMR $\left(101 \mathrm{MHz}, \mathrm{CDCl}_{3}\right) \delta 173.43,163.50,146.86,138.16,134.34,99.02$, 66.03, 48.78, 35.18, 31.84, 29.24, 28.61, 25.90, 22.72, 18.55, 14.20. HRMS (ESI): calcd for $\mathrm{C}_{19} \mathrm{H}_{27} \mathrm{I}_{2} \mathrm{NO}_{3} \mathrm{Na}[\mathrm{M}+\mathrm{Na}]^{+} 593.9973$ found 593.9945 .

\section{(S)-Octyl-2-(4-methyl-3,5-bis(2-(trimethylsilyl)ethynyl)benzamido)propanoate (11f).}

To a $100 \mathrm{~mL}$ two-necked flask charged with $\mathbf{3 f}(5.7 \mathrm{~g}, 10.0 \mathrm{mmol}), \mathrm{Pd}\left(\mathrm{PPh}_{3}\right)_{2} \mathrm{Cl}_{2}(214.5$ $\mathrm{mg}, 0.3 \mathrm{mmol})$ and $\mathrm{CuI}(114.3 \mathrm{mg}, 0.6 \mathrm{mmol})$ under argon, $40 \mathrm{~mL}$ THF and $20 \mathrm{~mL} \mathrm{Et}_{3} \mathrm{~N}$ were added via syringe. The solution was stirred for $10 \mathrm{~min}$ and then $5.6 \mathrm{~mL}$ ethynyltrimethylsilane $(3.9 \mathrm{~g}, 40 \mathrm{mmol})$ was injected into the solution. The mixture was stirred overnight at r.t. After removing the solvent under reduced pressure, the residue was purified by column chromatography (petroleum ether/acetone $=30 / 1$ ) to afforda yellow oil (4.2g, 82\%). ${ }^{1} \mathbf{H}$ NMR (400 MHz, $\left.\mathrm{CDCl}_{3}\right) \delta 7.54$ (s, $\left.2 \mathrm{H}\right), 6.64(\mathrm{~d}, J=7.3 \mathrm{~Hz}$, 1H), 4.49 (p, $J=7.1 \mathrm{~Hz}, 1 \mathrm{H}), 3.97-3.83(\mathrm{~m}, 2 \mathrm{H}), 2.30(\mathrm{~s}, 3 \mathrm{H}), 1.45-1.31(\mathrm{~m}, 2 \mathrm{H}), 1.24$ (d, $J=7.2 \mathrm{~Hz}, 3 \mathrm{H}), 1.17-0.88(\mathrm{~m}, 10 \mathrm{H}), 0.61(\mathrm{t}, J=6.7 \mathrm{~Hz}, 3 \mathrm{H}), 0.00(\mathrm{~d}, J=3.9 \mathrm{~Hz}$, 18H). ${ }^{13}$ C NMR (101 MHz, $\left.\mathrm{CDCl}_{3}\right) \delta 173.27,165.28,146.35,131.34,130.72,123.93$, $102.61,99.75,65.80,48.66,31.80,29.19,28.57,26.95,25.84,22.67,19.22,18.63,14.13$. HRMS (ESI): calcd for $\mathrm{C}_{29} \mathrm{H}_{45} \mathrm{NO}_{3} \mathrm{Si}_{2} \mathrm{Na}[\mathrm{M}+\mathrm{Na}]^{+} 534.2830$ found 534.2804 .

(S)-Octyl-2-(4-methyl-3,5-diethynylbenzamido)propanoate (2f). A solution of $11 \mathrm{f}$ (3.1g, $6.1 \mathrm{mmol}$ ) in $30 \mathrm{~mL}$ THF was added a solution of tetrabutylammonium fluoride (4.8g, $18.1 \mathrm{mmol})$ in $10 \mathrm{~mL}$ THF at r.t. The mixture was stirred for 30min. After removing the solvent under reduced pressure, the residue was redissolved in $30 \mathrm{~mL}$ $\mathrm{CHCl}_{3}$ and washed with water and brine. Then the organic layer was dried over anhydrous $\mathrm{Na}_{2} \mathrm{SO}_{4}$ and the solvent was removed under reduced pressure. The crude product was purified by column chromatography (petroleum ether/acetone $=10 / 1$ ) to 
afford a white solid (2.0g, 93\%). ${ }^{1} \mathbf{H}$ NMR $\left(400 \mathrm{MHz}, \mathrm{CDCl}_{3}\right) \delta 7.87$ (s, 2H), 6.75 (d, $J=$ $7.2 \mathrm{~Hz}, 1 \mathrm{H}), 4.76$ (p, J=7.1 Hz, 1H), $4.27-4.10$ (m, 2H), 3.34 (s, 2H), $2.62(\mathrm{~s}, 3 \mathrm{H})$, $1.74-1.62(\mathrm{~m}, 2 \mathrm{H}), 1.52(\mathrm{~d}, J=7.1 \mathrm{~Hz}, 3 \mathrm{H}), 1.47-1.13(\mathrm{~m}, 10 \mathrm{H}), 0.93-0.83(\mathrm{t}, J=$ $6.7 \mathrm{~Hz}, 3 \mathrm{H}) .{ }^{13} \mathbf{C}$ NMR $\left(101 \mathrm{MHz}, \mathrm{CDCl}_{3}\right) \delta 173.32,165.13,147.03,131.70,131.46$, $123.27,82.50,81.25,66.02,48.78,31.89,29.28,28.65,25.94,22.76,19.27,18.84,14.22$. HRMS (ESI): calcd for $\mathrm{C}_{23} \mathrm{H}_{29} \mathrm{NO}_{3} \mathrm{Na}[\mathrm{M}+\mathrm{Na}]+390.2040$ found 390.2052 .

Compound 4h. To a $100 \mathrm{~mL}$ two-necked flask charged with $\mathbf{3 a}(557.2 \mathrm{mg}, 1.0 \mathrm{mmol}), \mathbf{2 f}$ (183.7 $\mathrm{mg}, 0.50 \mathrm{mmol}), \mathrm{Pd}_{2}(\mathrm{dba})_{3}(13.7 \mathrm{mg}, 0.015 \mathrm{mmol})$ and $\mathrm{CuI}(5.7 \mathrm{mg}, 0.030 \mathrm{mmol})$, $\mathrm{PPh}_{3}(39.8 \mathrm{mg}, 0.15 \mathrm{mmol})$ under argon, $50 \mathrm{~mL}$ degassed $\mathrm{Et}_{3} \mathrm{~N}$ was added via syringe. The mixture was stirred at $75^{\circ} \mathrm{C}$ for $12 \mathrm{~h}$, and then removed the solvent under reduced pressure. The residue was purified by column chromatography $\left(\mathrm{CHCl}_{3} / \mathrm{THF}=60 / 1\right)$ to afford a light yellow solid (168.9 mg, 28\%). ${ }^{1} \mathbf{H}$ NMR $\left(400 \mathrm{MHz}, \mathrm{CDCl}_{3}\right) \delta 8.12(\mathrm{t}, J=$ $1.6 \mathrm{~Hz}, 2 \mathrm{H}), 8.01$ (t, $J=1.6 \mathrm{~Hz}, 2 \mathrm{H}), 7.92(\mathrm{~s}, 2 \mathrm{H}), 7.90$ (t, $J=1.5 \mathrm{~Hz}, 2 \mathrm{H}), 6.96(\mathrm{~d}, J=$ $7.4 \mathrm{~Hz}, 1 \mathrm{H}), 6.93-6.82(\mathrm{~m}, 2 \mathrm{H}), 4.81(\mathrm{~m}, 3 \mathrm{H}), 4.20$ (m, 6H), $2.70(\mathrm{~s}, 3 \mathrm{H}), 1.67$ (m, 6H), $1.55(\mathrm{~d}, J=7.1 \mathrm{~Hz}, 9 \mathrm{H}), 1.41-1.17(\mathrm{~m}, 30 \mathrm{H}), 0.88(\mathrm{t}, J=6.4 \mathrm{~Hz}, 9 \mathrm{H}) .{ }^{13} \mathbf{C}$ NMR $(101$ $\left.\mathrm{MHz}, \mathrm{CDCl}_{3}\right) \delta 173.51,173.31,165.03,164.52,145.65,142.72,136.24,135.69,132.10$, $131.58,131.08,129.42,125.06,123.41,93.78,91.75,89.08,65.96,65.91,48.76,48.65$, 31.76, 29.16, 28.52, 25.81, 22.63, 19.48, 18.52, 18.45, 14.10. MS (MALDI-TOF): calcd for $\mathrm{C}_{59} \mathrm{H}_{77} \mathrm{I}_{2} \mathrm{~N}_{3} \mathrm{O}_{9} \mathrm{Na}[\mathrm{M}+\mathrm{Na}]^{+} 1248.3641$ found 1248.6861 .

Compound 5h. To a $50 \mathrm{~mL}$ two-necked flask charged with compound $\mathbf{4 h}(245.2 \mathrm{mg}$, $0.20 \mathrm{mmol}), \mathrm{Pd}\left(\mathrm{PPh}_{3}\right)_{2} \mathrm{Cl}_{2}(4.3 \mathrm{mg}, 0.006 \mathrm{mmol})$ and $\mathrm{CuI}(2.3 \mathrm{mg}, 0.012 \mathrm{mmol})$ under argon, $15 \mathrm{~mL}$ THF and $8 \mathrm{~mL} \mathrm{Et}_{3} \mathrm{~N}$ were added via syringe. The solution was stirred for $10 \mathrm{~min}$ and then $0.11 \mathrm{mLethynyltrimethylsilane}(78.4 \mathrm{mg}, 0.8 \mathrm{mmol})$ was injected into the solution. The mixture was stirred overnight at r.t.. After removing the solvent under reduced pressure, the residue was purified by column chromatography $\left(\mathrm{CHCl}_{3} / \mathrm{THF}=\right.$ 90/1) to afford a yellow oil (219.1 mg, 94\%). $\left.{ }^{1} \mathbf{H} \mathbf{~ N M R ~ ( 4 0 0 ~ M H z , ~} \mathrm{CDCl}_{3}\right) \delta 7.93(\mathrm{~s}, 2 \mathrm{H})$, $7.90(\mathrm{~d}, J=1.6 \mathrm{~Hz}, 2 \mathrm{H}), 7.85(\mathrm{t}, J=1.7 \mathrm{~Hz}, 2 \mathrm{H}), 7.71(\mathrm{t}, J=1.5 \mathrm{~Hz}, 2 \mathrm{H}), 7.31(\mathrm{~d}, J=7.4$ 
$\mathrm{Hz}, 1 \mathrm{H}), 7.17(\mathrm{~d}, J=7.3 \mathrm{~Hz}, 2 \mathrm{H}), 4.82(\mathrm{~m}, 3 \mathrm{H}), 4.28-4.02(\mathrm{~m}, 6 \mathrm{H}), 2.66(\mathrm{~s}, 3 \mathrm{H}), 1.69-$ $1.57(\mathrm{~m}, 6 \mathrm{H}), 1.53(\mathrm{~d}, J=7.2 \mathrm{~Hz}, 9 \mathrm{H}), 1.37-1.16(\mathrm{~m}, 30 \mathrm{H}), 0.87-0.77(\mathrm{~m}, 9 \mathrm{H}), 0.25(\mathrm{~s}$, 18H). ${ }^{13}$ C NMR $\left(101 \mathrm{MHz}, \mathrm{CDCl}_{3}\right) \delta 173.63,173.43,165.46,165.35,145.79,137.52$, $134.63,131.73,131.18,130.68,130.25,124.31,123.80,123.74,103.11,96.65,92.71$, $88.58,66.04,66.01,48.92,48.86,31.93 \quad 29.33,28.69$ (s, 8H), 25.97, 22.80, 19.60, 18.66, 14.27. MS (MALDI-TOF): calcd for $\mathrm{C}_{69} \mathrm{H}_{95} \mathrm{~N}_{3} \mathrm{O}_{9} \mathrm{Si}_{2} \mathrm{Na}[\mathrm{M}+\mathrm{Na}]^{+} 1188.6499$ found 1188.7687 .

Compound 6h. A solution of compound $\mathbf{5 h}(175.0 \mathrm{mg}, 0.15 \mathrm{mmol})$ in $10 \mathrm{~mL}$ THF was added a solution of tetrabutylammonium fluoride $(117.5 \mathrm{mg}, 0.45 \mathrm{mmol})$ in $3 \mathrm{~mL}$ THF at r.t.. The mixture was stirred for 30min. After removing the solvent under reduced pressure, the residue was redissolved in $15 \mathrm{~mL} \mathrm{CHCl}_{3}$ and washed with water and brine. Then the organic layer was dried over anhydrous $\mathrm{Na}_{2} \mathrm{SO}_{4}$ and the solvent was removed under reduced pressure. The crude product was purified by column chromatography $\left(\mathrm{CHCl}_{3} / \mathrm{THF}=40 / 1\right)$ to afford a light yellow solid $(144.5 \mathrm{mg}, 94 \%) .{ }^{1} \mathbf{H}$ NMR $(400 \mathrm{MHz}$, $\left.\mathrm{CDCl}_{3}\right) \delta 7.91(\mathrm{~m}, 4 \mathrm{H}), 7.88(\mathrm{t}, J=1.4 \mathrm{~Hz}, 2 \mathrm{H}), 7.72(\mathrm{t}, J=1.4 \mathrm{~Hz}, 2 \mathrm{H}), 7.26(\mathrm{~d}, J=7.4$ $\mathrm{Hz}, 1 \mathrm{H}), 7.16(\mathrm{~d}, J=7.3 \mathrm{~Hz}, 2 \mathrm{H}), 4.93-4.70(\mathrm{~m}, 3 \mathrm{H}), 4.23-4.13(\mathrm{~m}, 6 \mathrm{H}), 3.15(\mathrm{~s}, 2 \mathrm{H})$, $2.66(\mathrm{~s}, 3 \mathrm{H}), 1.70-1.60(\mathrm{~m}, 6 \mathrm{H}), 1.54(\mathrm{~d}, J=7.1 \mathrm{~Hz}, 9 \mathrm{H}), 1.35-1.17(\mathrm{~m}, 30 \mathrm{H}), 0.86(\mathrm{~m}$, 9H). ${ }^{13} \mathbf{C}$ NMR $\left(101 \mathrm{MHz}, \mathrm{CDCl}_{3}\right) \delta 173.43,173.23,165.17,165.15,145.64,137.52$, $134.62,131.59,131.02,130.71,130.37,123.75,123.51,123.15,92.40,88.56,81.76$, $79.02,65.88,48.75,48.67,31.74,29.14,28.51,25.80,22.61,19.40,18.45,14.06 . \mathrm{MS}$ (MALDI-TOF): calcd for $\mathrm{C}_{63} \mathrm{H}_{79} \mathrm{~N}_{3} \mathrm{O}_{9} \mathrm{Na}[\mathrm{M}+\mathrm{Na}]+1044.5709$ found 1044.6471.

Macrocycle1h. To a $100 \mathrm{~mL}$ two-necked flask charged with $\mathrm{Pd}_{2}(\mathrm{dba})_{3}(22.6 \mathrm{mg}, 0.025$ $\mathrm{mmol})$ and $\mathrm{CuI}(9.4 \mathrm{mg}, 0.049 \mathrm{mmol}), \mathrm{PPh}_{3}(65.6 \mathrm{mg}, 0.25 \mathrm{mmol})$ under argon, $20 \mathrm{~mL}$ degassed $\mathrm{Et}_{3} \mathrm{~N}$ and $40 \mathrm{~mL}$ degassed $\mathrm{DMF}$ were added via syringe. The solution was stirred at $85^{\circ} \mathrm{C}$ for $10 \mathrm{~min}$. A solution of compound $4 \mathbf{a}(60.6 \mathrm{mg}, 0.050 \mathrm{mmol})$ and compound $6 \mathbf{h}(50.8 \mathrm{mg}, 0.050 \mathrm{mmol})$ in $10 \mathrm{~mL}$ DMF was slowly injected into the flask within $48 \mathrm{~h}$ by a syringe pump and then the solution was stirred for further $12 \mathrm{~h}$. The 
reaction mixture was concentrated in vacuo and purified by column chromatography $\left(\mathrm{CHCl}_{3} / \mathrm{THF} / \mathrm{AcOH}=25 / 1 / 0.5\right)$ to afford the crude product. The crude product was then washed with a few acetone and methanol to give the desired product (18.3 $\mathrm{mg}, 18 \%)$ as a white solid. ${ }^{1} \mathbf{H}$ NMR (400 MHz, DMF-d $\left.d_{7}\right) \delta 9.24(\mathrm{~d}, J=6.5 \mathrm{~Hz}, 5 \mathrm{H}), 9.17$ (d, $J=6.9 \mathrm{~Hz}$, $1 \mathrm{H}), 8.39(\mathrm{~s}, 3 \mathrm{H}), 8.33-8.23(\mathrm{~m}, 9 \mathrm{H}), 8.22-8.16(\mathrm{~m}, 2 \mathrm{H}), 8.15(\mathrm{~s}, 1 \mathrm{H}), 8.09(\mathrm{~m}, 2 \mathrm{H})$, $4.82-4.70(\mathrm{~m}, 6 \mathrm{H}), 4.27(\mathrm{~m}, 12 \mathrm{H}), 3.08(\mathrm{~s}, 3 \mathrm{H}), 1.81-1.63(\mathrm{~m}, 30 \mathrm{H}), 1.49-1.33(\mathrm{~m}$, $60 \mathrm{H}), 0.96(\mathrm{~m}, 18 \mathrm{H}) .{ }^{13} \mathbf{C}$ NMR $\left(101 \mathrm{MHz}, \mathrm{DMSO}-d_{6}\right) \delta 172.40,164.47,136.74,134.58$, $130.51,123.05,122.71,122.64,92.61,88.93,64.44,48.63,31.27,28.71,28.2125 .43$, 22.15, 16.64, 13.88. MS (MALDI-TOF): calcd for $\mathrm{C}_{121} \mathrm{H}_{152} \mathrm{~N}_{6} \mathrm{O}_{18} \mathrm{Na}[\mathrm{M}+\mathrm{Na}]^{+}$ 2000.1055 found 2000.0760 .

Macrocycle1i. To a $100 \mathrm{~mL}$ two-necked flask charged with $\mathrm{Pd}_{2}(\mathrm{dba})_{3}(22.6 \mathrm{mg}, 0.025$ $\mathrm{mmol})$ and $\mathrm{CuI}(9.4 \mathrm{mg}, 0.049 \mathrm{mmol}), \mathrm{PPh}_{3}(65.6 \mathrm{mg}, 0.25 \mathrm{mmol})$ under argon, $20 \mathrm{~mL}$ degassed $\mathrm{Et}_{3} \mathrm{~N}$ and $40 \mathrm{~mL}$ degassed $\mathrm{DMF}$ were added via syringe. The solution was stirred at $85^{\circ} \mathrm{C}$ for $10 \mathrm{~min}$. A solution of compound $4 \mathrm{~h}(61.3 \mathrm{mg}, 0.050 \mathrm{mmol})$ and compound 6h $(51.1 \mathrm{mg}, 0.050 \mathrm{mmol})$ in $10 \mathrm{~mL}$ DMF was slowly injected into the flask within $48 \mathrm{~h}$ by a syringe pump and then the solution was stirred for further $12 \mathrm{~h}$. The reaction mixture was concentrated in vacuo and purified by column chromatography $\left(\mathrm{CHCl}_{3} / \mathrm{THF} / \mathrm{AcOH}=25 / 1 / 0.5\right)$ to afford the crude product. The crude product was then washed with a few acetone and methanol to give the desired product(16.4 mg, 16\%) as a white solid. ${ }^{1} \mathrm{H}$ NMR (400 MHz, DMF- $\left.d_{7}\right) \delta 9.23$ (d, $\left.J=6.7 \mathrm{~Hz}, 4 \mathrm{H}\right), 9.16(\mathrm{~d}, J=6.8 \mathrm{~Hz}$, 2H), $8.41(\mathrm{~s}, 4 \mathrm{H}), 8.25(\mathrm{~m}, 8 \mathrm{H}), 8.13(\mathrm{~m}, 4 \mathrm{H}), 4.77(\mathrm{~m}, 6 \mathrm{H}), 4.28(\mathrm{~m}, 12 \mathrm{H}), 3.01(\mathrm{~s}, 4 \mathrm{H})$, $1.84-1.60(\mathrm{~m}, 30 \mathrm{H}), 1.53-1.30(\mathrm{~m}, 60 \mathrm{H}), 0.96(\mathrm{~m}, 18 \mathrm{H}) .{ }^{13} \mathbf{C}$ NMR $(101 \mathrm{MHz}$, DMSO- $\left.d_{6}\right) \delta 172.39,164.43,132.09,131.98,131.88,131.52,131.42,128.59,128.48$, $122.93,122.75,122.69,64.41,48.62,31.25,28.69,28.18,25.41,22.13,19.64,16.61$, 13.88. MS (MALDI-TOF): calcd for $\mathrm{C}_{122} \mathrm{H}_{154} \mathrm{~N}_{6} \mathrm{O}_{18} \mathrm{Na}[\mathrm{M}+\mathrm{Na}]+2014.1212$ found 2014.0349.

Compound 4f. To a $100 \mathrm{~mL}$ two-necked flask charged with $\mathbf{3 a}(557.2 \mathrm{mg}, 1.0 \mathrm{mmol}), \mathbf{2 c}$ 
(185.7 mg, $0.50 \mathrm{mmol}), \mathrm{Pd}_{2}(\mathrm{dba})_{3}(13.7 \mathrm{mg}, 0.015 \mathrm{mmol})$ and $\mathrm{CuI}(5.7 \mathrm{mg}, 0.030 \mathrm{mmol})$, $\mathrm{PPh}_{3}(39.8 \mathrm{mg}, 0.15 \mathrm{mmol})$ under argon, $50 \mathrm{~mL}$ degassed $\mathrm{Et}_{3} \mathrm{~N}$ was added via syringe. The mixture was stirred at $75^{\circ} \mathrm{C}$ for $12 \mathrm{~h}$, and then removed the solvent under reduced pressure. The residue was purified by column chromatography $\left(\mathrm{CHCl}_{3} / \mathrm{THF}=50 / 1\right)$ to afford a light yellow solid(210.5 mg, 34\%). ${ }^{1} \mathbf{H}$ NMR $\left(400 \mathrm{MHz}, \mathrm{CDCl}_{3}\right) \delta 8.16(\mathrm{t}, J=1.5$ $\mathrm{Hz}, 2 \mathrm{H}), 8.02(\mathrm{t}, J=1.4 \mathrm{~Hz}, 2 \mathrm{H}), 7.96(\mathrm{~d}, J=6.1 \mathrm{~Hz}, 2 \mathrm{H}), 7.91(\mathrm{~d}, J=1.3 \mathrm{~Hz}, 2 \mathrm{H}), 7.08$ (d, $J=7.3 \mathrm{~Hz}, 1 \mathrm{H}), 7.01(\mathrm{~d}, J=7.3 \mathrm{~Hz}, 2 \mathrm{H}), 4.82(\mathrm{~m}, 3 \mathrm{H}), 4.26-4.14(\mathrm{~m}, 6 \mathrm{H}), 1.74-$ $1.62(\mathrm{~m}, 6 \mathrm{H}), 1.55(\mathrm{~m}, 9 \mathrm{H}), 1.28(\mathrm{~m}, 30 \mathrm{H}), 0.88(\mathrm{t}, J=6.8 \mathrm{~Hz}, 9 \mathrm{H}) .{ }^{13} \mathbf{C} \mathbf{N M R}(101 \mathrm{MHz}$, $\left.\mathrm{CDCl}_{3}\right) \delta 173.33,173.19,164.38,164.13,162.82,142.94,136.73,135.80,132.70,129.45$, $124.49,112.28,93.81,93.21,83.24,65.97,48.77,31.75,29.14,28.52,25.80,22.62$, 18.54, 18.52, 14.08. MS (MALDI-TOF): calcd for $\mathrm{C}_{58} \mathrm{H}_{74} \mathrm{FI}_{2} \mathrm{~N}_{3} \mathrm{O}_{9} \mathrm{Na}[\mathrm{M}+\mathrm{Na}]^{+}$ 1252.3391 found 1252.0112 .

Compound 5f. To a $50 \mathrm{~mL}$ two-necked flask charged with compound $\mathbf{4 f}$ ( $246.0 \mathrm{mg}, 0.20$ $\mathrm{mmol}), \mathrm{Pd}\left(\mathrm{PPh}_{3}\right)_{2} \mathrm{Cl}_{2}(4.3 \mathrm{mg}, 0.006 \mathrm{mmol})$ and $\mathrm{CuI}(2.3 \mathrm{mg}, 0.012 \mathrm{mmol})$ under argon, $15 \mathrm{~mL}$ THF and $8 \mathrm{~mL} \mathrm{Et}_{3} \mathrm{~N}$ were added via syringe. The solution was stirred for $10 \mathrm{~min}$ and then $0.11 \mathrm{mLethynyltrimethylsilane}(78.4 \mathrm{mg}, 0.8 \mathrm{mmol}$ ) was injected into the solution. The mixture was stirred overnight at r.t.. After removing the solvent under reduced pressure, the residue was purified by column chromatography $\left(\mathrm{CHCl}_{3} / \mathrm{THF}=\right.$ 90/1) to afford a yellow oil(211.1 mg, 90\%). ${ }^{1} \mathbf{H}$ NMR $\left(400 \mathrm{MHz}, \mathrm{CDCl}_{3}\right) \delta 7.98(\mathrm{~d}, J=$ $6.1 \mathrm{~Hz}, 2 \mathrm{H}), 7.92$ (s, 2H), 7.89 (s, 2H), 7.76 (s, 2H), 7.18 (d, $J=7.3 \mathrm{~Hz}, 1 \mathrm{H}), 7.04$ (d, $J=$ $7.3 \mathrm{~Hz}, 2 \mathrm{H}), 4.87-4.76(\mathrm{~m}, 3 \mathrm{H}), 4.24-4.11(\mathrm{~m}, 6 \mathrm{H}), 1.73-1.61(\mathrm{~m}, 6 \mathrm{H}), 1.55(\mathrm{~m}, 9 \mathrm{H})$, $1.36-1.23(\mathrm{~m}, 30 \mathrm{H}), 0.87(\mathrm{t}, J=6.7 \mathrm{~Hz}, 9 \mathrm{H}), 0.26(\mathrm{~s}, 18 \mathrm{H}) .{ }^{13} \mathbf{C} \mathbf{N M R}(101 \mathrm{MHz}$, $\left.\mathrm{CDCl}_{3}\right) \delta 173.25,173.19,165.13,164.26,162.72,137.58,134.56,132.63,130.86,130.20$, 130.07, 124.31, 123.08, 112.33 (d, $J=16.3 \mathrm{~Hz}), 102.78,96.68,94.04,82.58,65.88$, $48.76(\mathrm{~d}, J=6.4 \mathrm{~Hz}), 31.73,29.12,28.51,25.78,22.60,18.55,18.49,14.05,-0.22 . \mathrm{MS}$ (MALDI-TOF): calcd for $\mathrm{C}_{68} \mathrm{H}_{92} \mathrm{FN}_{3} \mathrm{O}_{9} \mathrm{Si}_{2} \mathrm{Na}[\mathrm{M}+\mathrm{Na}]^{+} 1192.6248$ found 1192.4454.

Compound 6f. A solution of compound $\mathbf{5 f}(175.6 \mathrm{mg}, 0.15 \mathrm{mmol})$ in $10 \mathrm{~mL}$ THF was 
added a solution of tetrabutylammonium fluoride $(117.5 \mathrm{mg}, 0.45 \mathrm{mmol})$ in $3 \mathrm{~mL}$ THF at r.t.. The mixture was stirred for $30 \mathrm{~min}$. After removing the solvent under reduced pressure, the residue was redissolved in $15 \mathrm{~mL} \mathrm{CHCl}_{3}$ and washed with water and brine. Then the organic layer was dried over anhydrous $\mathrm{Na}_{2} \mathrm{SO}_{4}$ and the solvent was removed under reduced pressure. The crude product was purified by column chromatography $\left(\mathrm{CHCl}_{3} / \mathrm{THF}=40 / 1\right)$ to afford a light yellow solid $(142.8 \mathrm{mg}, 93 \%) .{ }^{1} \mathbf{H} \mathbf{~ N M R}(400 \mathrm{MHz}$, $\left.\mathrm{CDCl}_{3}\right) \delta 7.97(\mathrm{~d}, J=6.1 \mathrm{~Hz}, 2 \mathrm{H}), 7.94-7.90(\mathrm{~m}, 4 \mathrm{H}), 7.76(\mathrm{t}, J=1.4 \mathrm{~Hz}, 2 \mathrm{H}), 7.24(\mathrm{~d}$, $J=7.4 \mathrm{~Hz}, 1 \mathrm{H}), 7.11(\mathrm{~d}, J=7.4 \mathrm{~Hz}, 2 \mathrm{H}), 4.87-4.77(\mathrm{~m}, 3 \mathrm{H}), 4.24-4.11(\mathrm{~m}, 6 \mathrm{H}), 3.16$ (s, 2H), $1.71-1.61(\mathrm{~m}, 6 \mathrm{H}), 1.55(\mathrm{~m}, 9 \mathrm{H}), 1.35-1.21(\mathrm{~m}, 30 \mathrm{H}), 0.87$ (t, $J=6.8 \mathrm{~Hz}, 9 \mathrm{H})$. ${ }^{13}$ C NMR $\left(101 \mathrm{MHz}, \mathrm{CDCl}_{3}\right) \delta$ 173.32, 173.20, 165.04, 164.26, $164.06(\mathrm{~d}, J=263.8 \mathrm{~Hz})$, $137.71,134.67,132.70,131.17,130.41,130.21,125.44,123.29,123.19,112.25$ (d, $J=$ $16.3 \mathrm{~Hz}), 93.83,82.72,81.63,79.1365 .91$ (d, $J=2.8 \mathrm{~Hz}), 48.76$ (d, $J=3.9 \mathrm{~Hz}), 31.73$, $31.73,29.13,29.13,28.51,28.51,25.79,25.79,22.60,22.60,18.48,18.46$ (d, $J=5.5 \mathrm{~Hz}$ ), 18.43, 14.05, 14.05. MS (MALDI-TOF): calcd for $\mathrm{C}_{62} \mathrm{H}_{76} \mathrm{FN}_{3} \mathrm{O}_{9} \mathrm{Na}[\mathrm{M}+\mathrm{Na}]^{+} 1048.5458$ found 1048.3972 .

Compound 4g. To a $100 \mathrm{~mL}$ two-necked flask charged with $\mathbf{3 c}(575.2 \mathrm{mg}, 1.0 \mathrm{mmol}), \mathbf{2 a}$ (176.8 $\mathrm{mg}, 0.50 \mathrm{mmol}), \mathrm{Pd}_{2}(\mathrm{dba})_{3}(13.7 \mathrm{mg}, 0.015 \mathrm{mmol})$ and $\mathrm{CuI}(5.7 \mathrm{mg}, 0.030 \mathrm{mmol})$, $\mathrm{PPh}_{3}$ (39.8 mg, $0.15 \mathrm{mmol}$ ) under argon, $50 \mathrm{~mL}$ degassed $\mathrm{Et}_{3} \mathrm{~N}$ was added via syringe. The mixture was stirred at $75^{\circ} \mathrm{C}$ for $12 \mathrm{~h}$, and then removed the solvent under reduced pressure. The residue was purified by column chromatography $\left(\mathrm{CHCl}_{3} / \mathrm{THF}=50 / 1\right)$ to afford a light yellow solid (236.5 mg, 38\%). ${ }^{1} \mathbf{H}$ NMR (400 MHz, $\left.\mathrm{CDCl}_{3}\right) \delta 8.21$ (dd, $J=$ 5.5, 2.1 Hz, 2H), 7.96 (d, $J=1.4 \mathrm{~Hz}, 2 \mathrm{H}), 7.91$ (dd, $J=6.0,2.1 \mathrm{~Hz}, 2 \mathrm{H}), 7.79(\mathrm{~s}, 1 \mathrm{H})$, 7.30 (d, $J=7.5 \mathrm{~Hz}, 1 \mathrm{H}), 7.15$ (d, $J=7.3 \mathrm{~Hz}, 2 \mathrm{H}), 4.84(\mathrm{~m}, 3 \mathrm{H}), 4.25-4.13(\mathrm{~m}, 6 \mathrm{H}), 1.74$ $-1.62(\mathrm{~m}, 6 \mathrm{H}), 1.56(\mathrm{~m}, 9 \mathrm{H}), 1.41-1.21(\mathrm{~m}, 30 \mathrm{H}), 0.93-0.83(\mathrm{~m}, 9 \mathrm{H}) .{ }^{13} \mathbf{C} \mathbf{N M R}(101$ $\left.\mathrm{MHz}, \mathrm{CDCl}_{3}\right) \delta 173.39,173.33,164.98,163.68,163.36(\mathrm{~d}, J=255.8 \mathrm{~Hz}), 138.97,$,137.17 , 134.68, 132.39, 131.40 (d, $J=4.0 \mathrm{~Hz}), 130.78,123.27,111.83$ (d, $J=19.2 \mathrm{~Hz}), 99.99$, $94.01,82.77,81.73,81.46,65.97,48.80,31.74,29.14,28.52,25.80,22.61,18.41,14.07$. 
MS (MALDI-TOF): calcd for $\mathrm{C}_{58} \mathrm{H}_{73} \mathrm{~F}_{2} \mathrm{I}_{2} \mathrm{~N}_{3} \mathrm{O}_{9} \mathrm{Na}[\mathrm{M}+\mathrm{Na}]+1270.3296$ found 1270.2638 .

Compound 5g. To a $50 \mathrm{~mL}$ two-necked flask charged with compound $\mathbf{4 g}$ (249.6 mg, $0.20 \mathrm{mmol}), \mathrm{Pd}\left(\mathrm{PPh}_{3}\right)_{2} \mathrm{Cl}_{2}(4.3 \mathrm{mg}, 0.006 \mathrm{mmol})$ and $\mathrm{CuI}(2.3 \mathrm{mg}, 0.012 \mathrm{mmol})$ under argon, $15 \mathrm{~mL}$ THF and $8 \mathrm{~mL} \mathrm{Et}_{3} \mathrm{~N}$ were added via syringe. The solution was stirred for $10 \mathrm{~min}$ and then $0.11 \mathrm{mLethynyltrimethylsilane}(78.4 \mathrm{mg}, 0.8 \mathrm{mmol})$ was injected into the solution. The mixture was stirred overnight at r.t.. After removing the solvent under reduced pressure, the residue was purified by column chromatography $\left(\mathrm{CHCl}_{3} / \mathrm{THF}=\right.$ 80/1) to afford a yellow oil (215.7 mg, 91\%). ${ }^{\mathbf{1}} \mathbf{H} \mathbf{~ N M R}\left(400 \mathrm{MHz}, \mathrm{CDCl}_{3}\right) \delta 7.99(\mathrm{~d}, J=$ $1.4 \mathrm{~Hz}, 2 \mathrm{H}), 7.92(\mathrm{~m}, 4 \mathrm{H}), 7.82$ (t, $J=1.3 \mathrm{~Hz}, 1 \mathrm{H}), 7.21$ (d, $J=7.4 \mathrm{~Hz}, 1 \mathrm{H}), 7.02$ (d, $J=$ $7.3 \mathrm{~Hz}, 2 \mathrm{H}), 4.82(\mathrm{~m}, 3 \mathrm{H}), 4.26-4.11(\mathrm{~m}, 6 \mathrm{H}), 1.72-1.60(\mathrm{~m}, 6 \mathrm{H}), 1.55(\mathrm{~m}, 9 \mathrm{H}), 1.29$ $(\mathrm{m}, 30 \mathrm{H}), 0.91-0.83(\mathrm{~m}, 9 \mathrm{H}), 0.28(\mathrm{~s}, 18 \mathrm{H}) .{ }^{13} \mathbf{C}$ NMR $\left(101 \mathrm{MHz}, \mathrm{CDCl}_{3}\right) \delta 173.24$, $173.19,165.03,164.50$ (d, $J=263.6 \mathrm{~Hz}), 164.32$, 137.23, 134.76, 133.05, 132.19, 130.66, $130.03(\mathrm{~d}, J=4.0 \mathrm{~Hz}), 123.43,112.86(\mathrm{~d}, J=16.5 \mathrm{~Hz}), 112.07$ (d, $J=16.5 \mathrm{~Hz})$, $102.45,96.01,93.66,87.92,86.79,82.95,65.90,48.76,31.73,29.12,28.50,25.78,22.60$, $18.51(\mathrm{~d}, J=3.3 \mathrm{~Hz}), 14.05,-0.31$. MS (MALDI-TOF): calcd for $\mathrm{C}_{68} \mathrm{H}_{91} \mathrm{~F}_{2} \mathrm{~N}_{3} \mathrm{O}_{9} \mathrm{Si}_{2} \mathrm{Na}[\mathrm{M}$ $+\mathrm{Na}]+1210.6154$ found 1210.4253 .

Compound 6g. A solution of compound $5 \mathrm{~g}(178.3 \mathrm{mg}, 0.15 \mathrm{mmol})$ in $10 \mathrm{~mL}$ THF was added a solution of tetrabutylammonium fluoride $(117.5 \mathrm{mg}, 0.45 \mathrm{mmol})$ in $3 \mathrm{~mL}$ THF at r.t.. The mixture was stirred for 30min. After removing the solvent under reduced pressure, the residue was redissolved in $15 \mathrm{~mL} \mathrm{CHCl}_{3}$ and washed with water and brine. Then the organic layer was dried over anhydrous $\mathrm{Na}_{2} \mathrm{SO}_{4}$ and the solvent was removed under reduced pressure. The crude product was purified by column chromatography $\left(\mathrm{CHCl}_{3} / \mathrm{THF}=40 / 1\right)$ to afford a light yellow solid $(147.5 \mathrm{mg}, 94 \%) .{ }^{1} \mathbf{H}$ NMR $(400 \mathrm{MHz}$, $\left.\mathrm{CDCl}_{3}\right) \delta 7.98(\mathrm{~d}, J=1.4 \mathrm{~Hz}, 2 \mathrm{H}), 7.94(\mathrm{~d}, J=6.1 \mathrm{~Hz}, 4 \mathrm{H}), 7.81(\mathrm{~s}, 1 \mathrm{H}), 7.30(\mathrm{~d}, J=7.5$ Hz, 1H), 7.12 (d, J=7.3 Hz, 2H), $4.82(\mathrm{~m}, 3 \mathrm{H}), 4.25-4.12(\mathrm{~m}, 6 \mathrm{H}), 3.38(\mathrm{~s}, 2 \mathrm{H}), 1.74-$ $1.60(\mathrm{~m}, 6 \mathrm{H}), 1.55(\mathrm{~m}, 9 \mathrm{H}), 1.29(\mathrm{~m}, 30 \mathrm{H}), 0.92-0.82(\mathrm{~m}, 9 \mathrm{H}) .{ }^{\mathbf{1 3}} \mathbf{C}$ NMR $(101 \mathrm{MHz}$, 
$\left.\mathrm{CDCl}_{3}\right) \delta 173.33,173.23,165.04,164.74(\mathrm{~d}, J=264.0 \mathrm{~Hz}), 164.23,137.19,134.69$, 133.36, 132.65, 130.77, 130.15 (d, $J=4.2 \mathrm{~Hz}), 123.32,112.17$ (d, $J=16.4 \mathrm{~Hz}), 111.76$ (d, $J=16.3 \mathrm{~Hz}$ ), 93.82, 84.11, 82.76, 75.41, 65.92, 48.78, 31.73, 29.13, 28.51, 25.79, 22.60, 18.44, 14.05. MS (MALDI-TOF): calcd for $\mathrm{C}_{62} \mathrm{H}_{75} \mathrm{~F}_{2} \mathrm{~N}_{3} \mathrm{O}_{9} \mathrm{Na}[\mathrm{M}+\mathrm{Na}]+$ 1066.5364 found 1066.5379 .

Macrocycle1f. To a $100 \mathrm{~mL}$ two-necked flask charged with $\operatorname{Pd}_{2}(\mathrm{dba})_{3}(22.6 \mathrm{mg}, 0.025$ $\mathrm{mmol})$ and $\mathrm{CuI}(9.4 \mathrm{mg}, 0.049 \mathrm{mmol}), \mathrm{PPh}_{3}(65.6 \mathrm{mg}, 0.25 \mathrm{mmol})$ under argon, $20 \mathrm{~mL}$ degassed $\mathrm{Et}_{3} \mathrm{~N}$ and $40 \mathrm{~mL}$ degassed DMF were added via syringe. The solution was stirred at $85^{\circ} \mathrm{C}$ for $10 \mathrm{~min}$. A solution of compound $4 \mathbf{f}(60.0 \mathrm{mg}, 0.050 \mathrm{mmol})$ and compound $6 \mathrm{~g}$ (50.8 $\mathrm{mg}, 0.050 \mathrm{mmol})$ in $10 \mathrm{~mL}$ DMF was slowly injected into the flask within $48 \mathrm{~h}$ by a syringe pump and then the solution was stirred for further $12 \mathrm{~h}$. The reaction mixture was concentrated in vacuo and purified by column chromatography $\left(\mathrm{CHCl}_{3} / \mathrm{THF} / \mathrm{AcOH}=25 / 1 / 0.5\right)$ to afford the crude product. The crude product was then washed with a few acetone and methanol to give the desired product $(17.7 \mathrm{mg}, 18 \%)$ as a light yellow solid. ${ }^{1}$ H NMR (400 MHz, DMF- $\left.d_{7}\right) \delta 9.08(\mathrm{~d}, J=6.5 \mathrm{~Hz}, 6 \mathrm{H}), 8.19(\mathrm{~d}, J=$ $6.4 \mathrm{~Hz}, 3 \mathrm{H}), 8.13$ (d, $J=1.4 \mathrm{~Hz}, 6 \mathrm{H}), 8.02-7.99$ (m, 6H), $4.70-4.59$ (m, 6H), $4.23-$ $4.09(\mathrm{~m}, 12 \mathrm{H}), 1.65(\mathrm{~m}, 12 \mathrm{H}), 1.61(\mathrm{t}, J=7.1 \mathrm{~Hz}, 18 \mathrm{H}), 1.31(\mathrm{~m}, 60 \mathrm{H}), 0.85$ (t, $J=6.9$ $\mathrm{Hz}, 18 \mathrm{H}) .{ }^{13} \mathbf{C}$ NMR $\left(101 \mathrm{MHz}, \mathrm{DMF}-d_{7}\right) \delta 172.80,172.74,166.09,123.66,122.46$, 89.22, 82.77, 74.55, 64.73 (s, -4H), 49.42, 31.68, 29.14, 29.12, 25.80, 22.48, 16.56, 13.65. MS (MALDI-TOF): calcd for $\mathrm{C}_{120} \mathrm{H}_{147} \mathrm{~F}_{3} \mathrm{~N}_{6} \mathrm{O}_{18} \mathrm{Na}[\mathrm{M}+\mathrm{Na}]+2040.0616$ found 2040.5899 .

Macrocycle1g. To a $100 \mathrm{~mL}$ two-necked flask charged with $\operatorname{Pd}_{2}(\mathrm{dba})_{3}(22.6 \mathrm{mg}, 0.025$ $\mathrm{mmol})$ and $\mathrm{CuI}(9.4 \mathrm{mg}, 0.049 \mathrm{mmol}), \mathrm{PPh}_{3}(65.6 \mathrm{mg}, 0.25 \mathrm{mmol})$ under argon, $20 \mathrm{~mL}$ degassed $\mathrm{Et}_{3} \mathrm{~N}$ and $40 \mathrm{~mL}$ degassed DMF were added via syringe. The solution was stirred at $85^{\circ} \mathrm{C}$ for $10 \mathrm{~min}$. A solution of compound $\mathbf{4 f}(61.5 \mathrm{mg}, 0.050 \mathrm{mmol})$ and compound $\mathbf{6 f}(51.3 \mathrm{mg}, 0.050 \mathrm{mmol})$ in $10 \mathrm{~mL}$ DMF was slowly injected into the flask within $48 \mathrm{~h}$ by a syringe pump and then the solution was stirred for further $12 \mathrm{~h}$. The 
reaction mixture was concentrated in vacuo and purified by column chromatography $\left(\mathrm{CHCl}_{3} / \mathrm{THF} / \mathrm{AcOH}=25 / 1 / 0.5\right)$ to afford the crude product. The crude product was then washed with a few acetone and methanol to give the desired product $(15.8 \mathrm{mg}, 16 \%)$ as a light yellow solid. ${ }^{1}$ H NMR (400 MHz, DMSO-d $\left.d_{6}\right) \delta 8.97$ (s, 6H), 8.13 (d, $\left.J=6.1 \mathrm{~Hz}, 4 \mathrm{H}\right)$, $8.06(\mathrm{~s}, 8 \mathrm{H}), 7.90(\mathrm{~s}, 4 \mathrm{H}), 4.53-4.43(\mathrm{~m}, 6 \mathrm{H}), 4.15-4.00(\mathrm{~m}, 12 \mathrm{H}), 1.57(\mathrm{~m}, 12 \mathrm{H}), 1.46$ $(\mathrm{d}, J=7.2 \mathrm{~Hz}, 18 \mathrm{H}), 1.34-1.13(\mathrm{~m}, 60 \mathrm{H}), 0.81(\mathrm{t}, J=6.8 \mathrm{~Hz}, 18 \mathrm{H}) .{ }^{13} \mathbf{C}$ NMR $(101$ MHz, DMF- $\left.d_{7}\right) \delta 172.95,172.89,165.09,137.08,136.24,133.48,131.04,123.27(\mathrm{~d}, J=$ 64.0 Hz), 111.81, 111.67, 94.89, 89.34, 82.63, 64.90, 49.50, 31.86, 29.31, 28.78, 25.98, 22.66, 16.74, 13.83. MS (MALDI-TOF): calcd for $\mathrm{C}_{120} \mathrm{H}_{148} \mathrm{~F}_{2} \mathrm{~N}_{6} \mathrm{O}_{18} \mathrm{Na}[\mathrm{M}+\mathrm{Na}]+$ 2022.0710 found 2021.4785.

(S)-Octyl-2-(4-nitrobenzamido)propanoate (9). To a $250 \mathrm{~mL}$ round bottom flask was added 4-nitrobenzoic acid (10.0 g, $59.9 \mathrm{mmol}), 100 \mathrm{~mL}$ redistilled $\mathrm{CH}_{2} \mathrm{Cl}_{2}$ and $5.6 \mathrm{~mL}$ $(\mathrm{COCl})_{2}$ (oxalyl chloride) $(8.4 \mathrm{~g}, 66.1 \mathrm{mmol})$, then $0.1 \mathrm{~mL}$ DMF was added dropwise. The solution was stirred for $6 \mathrm{~h}$. After removing the solvent and the redundant $(\mathrm{COCl})_{2}$ under reduced pressure, the residue was resolve in $20 \mathrm{~mL}$ redistilled $\mathrm{CH}_{2} \mathrm{Cl}_{2}$ and added dropwise to a $250 \mathrm{~mL}$ ice-cooled round bottom flask containing $100 \mathrm{~mL}$ redistilled $\mathrm{CH}_{2} \mathrm{Cl}_{2}, 26 \mathrm{~mL} \mathrm{Et} 3 \mathrm{~N}(18.1 \mathrm{~g}, 17.9 \mathrm{mmol})$ and $(S)$-octyl-2-aminopropanoate hydrochloride $(14.9 \mathrm{~g}, 62.9 \mathrm{mmol})$. The mixture was stirred for $8 \mathrm{~h}$ at r.t.. The solution was washed with water and brine, and then concentrated in vacuo. The crude product was purified by column chromatography (petroleum ether/acetone $=6 / 1)$ to afford a white solid $(17.6 \mathrm{~g}$, $84 \%) .{ }^{1} \mathbf{H}$ NMR $\left(400 \mathrm{MHz}, \mathrm{CDCl}_{3}\right) \delta 8.28(\mathrm{~d}, J=8.7 \mathrm{~Hz}, 2 \mathrm{H}), 7.97(\mathrm{~d}, J=8.7 \mathrm{~Hz}, 2 \mathrm{H})$, $6.94(\mathrm{~d}, J=6.8 \mathrm{~Hz}, 1 \mathrm{H}), 4.78(\mathrm{~m}, 1 \mathrm{H}), 4.28-4.10(\mathrm{~m}, 2 \mathrm{H}), 1.72-1.62(\mathrm{~m}, 2 \mathrm{H}), 1.55(\mathrm{~d}$, $J=7.1 \mathrm{~Hz}, 3 \mathrm{H}), 1.37-1.23(\mathrm{~m}, 10 \mathrm{H}), 0.88(\mathrm{t}, J=6.7 \mathrm{~Hz}, 3 \mathrm{H}) .{ }^{13} \mathbf{C}$ NMR $(101 \mathrm{MHz}$, $\left.\mathrm{CDCl}_{3}\right) \delta 173.02,164.69,149.72,139.49,128.26,123.80,66.07,48.87,31.72,29.12$, 28.49, 25.77, 22.60, 18.56, 14.05. HRMS (ESI): calcd for $\mathrm{C}_{18} \mathrm{H}_{27} \mathrm{~N}_{2} \mathrm{O}_{5}[\mathrm{M}+\mathrm{H}]+$ 351.1914 found 351.1915 .

(S)-Octyl-2-(4-aminobenzamido)propanoate (10). To a $250 \mathrm{~mL}$ two-necked flask 
charged with $9(17.5 \mathrm{~g}, 50.0 \mathrm{mmol})$ and $1.8 \mathrm{~g}$ of $10 \% \mathrm{Pd} / \mathrm{Cunder}$ hydrogen, $100 \mathrm{~mL}$ $\mathrm{CH}_{3} \mathrm{OH}$ were added via syringe. The solution was stirred for $12 \mathrm{~h}$ and filtered. The filtration was then concentrated to afford a white solid (15.6g, 97\%) as the product. ${ }^{1} \mathbf{H}$ NMR $\left(400 \mathrm{MHz}, \mathrm{CDCl}_{3}\right) \delta 7.64(\mathrm{~d}, J=8.6 \mathrm{~Hz}, 2 \mathrm{H}), 6.66(\mathrm{~d}, J=8.6 \mathrm{~Hz}, 2 \mathrm{H}), 6.61(\mathrm{~d}, J=$ $7.2 \mathrm{~Hz}, 1 \mathrm{H}), 4.77(\mathrm{~m}, 1 \mathrm{H}), 4.16(\mathrm{~m}, 2 \mathrm{H}), 3.99(\mathrm{~s}, 2 \mathrm{H}), 1.73-1.60(\mathrm{~m}, 2 \mathrm{H}), 1.50$ (d, J= $7.1 \mathrm{~Hz}, 3 \mathrm{H}), 1.39-1.21(\mathrm{~m}, 10 \mathrm{H}), 0.88(\mathrm{t}, J=6.8 \mathrm{~Hz}, 3 \mathrm{H}) .{ }^{13} \mathbf{C} \mathbf{N M R}\left(101 \mathrm{MHz}, \mathrm{CDCl}_{3}\right)$ $\delta 173.63,166.45,149.76,128.82,123.54,114.09,65.64,48.41,31.74,29.14,28.52,25.80$, 22.61, 18.93, 14.06. HRMS (ESI): calcd for $\mathrm{C}_{18} \mathrm{H}_{29} \mathrm{~N}_{2} \mathrm{O}_{3}[\mathrm{M}+\mathrm{H}]+321.2173$ found 321.2172 .

(S)-Octyl-2-(4-amino-3,5-diiodobenzamido)propanoate (3g). To a $250 \mathrm{~mL}$ flask was added $10(15 \mathrm{~g}, 46.8 \mathrm{mmol})$ and $150 \mathrm{~mL}$ ethanol, followed with iodine $(26.2 \mathrm{~g}, 103.0$ $\mathrm{mmol})$ and $\mathrm{Ag}_{2} \mathrm{SO}_{4}(16.1 \mathrm{~g}, 51.5 \mathrm{mmol})$. The mixture was stirred for $2 \mathrm{~h}$ and filtered. The filtration was then concentrated to afford the crude product. The crude product was purified by column (petroleum ether/acetone $=4 / 1)$ to give the desired product $(13.1 \mathrm{~g}$, 49\%) as a white solid. ${ }^{1} \mathbf{H}$ NMR (400 MHz, $\left.\mathrm{CDCl}_{3}\right) \delta 8.08(\mathrm{~s}, 2 \mathrm{H}), 6.59(\mathrm{~d}, J=7.1 \mathrm{~Hz}$, 1H), $4.96(\mathrm{~s}, 2 \mathrm{H}), 4.74(\mathrm{p}, J=7.2 \mathrm{~Hz}, 1 \mathrm{H}), 4.25-4.10(\mathrm{~m}, 2 \mathrm{H}), 1.75-1.59(\mathrm{~m}, 2 \mathrm{H}), 1.50$ $(\mathrm{d}, J=7.1 \mathrm{~Hz}, 3 \mathrm{H}), 1.40-1.22(\mathrm{~m}, 10 \mathrm{H}), 0.89(\mathrm{t}, J=6.8 \mathrm{~Hz}, 3 \mathrm{H}) .{ }^{13} \mathbf{C}$ NMR $(101 \mathrm{MHz}$, $\left.\mathrm{CDCl}_{3}\right) \delta 173.42,163.59,148.84,138.47,126.27,79.96,65.84,48.59,31.75,29.14,28.52$, 25.81, 22.62, 18.72, 14.08. HRMS (ESI): calcd for $\mathrm{C}_{18} \mathrm{H}_{27} \mathrm{I}_{2} \mathrm{~N}_{2} \mathrm{O}_{3}[\mathrm{M}+\mathrm{H}]+573.0106$ found 573.0109 .

\section{(S)-Octyl-2-(4-methyl-3,5-bis(2-(trimethylsilyl)ethynyl)benzamido)propanoate (11g).}

To a $100 \mathrm{~mL}$ two-necked flask charged with $3 \mathrm{~g}(5.7 \mathrm{~g}, 10.0 \mathrm{mmol}), \mathrm{Pd}\left(\mathrm{PPh}_{3}\right)_{2} \mathrm{Cl}_{2}(214.5$ $\mathrm{mg}, 0.3 \mathrm{mmol})$ and $\mathrm{CuI}(114.3 \mathrm{mg}, 0.6 \mathrm{mmol})$ under argon, $40 \mathrm{~mL}$ THF and $20 \mathrm{~mL} \mathrm{Et}_{3} \mathrm{~N}$ were added via syringe. The solution was stirred for $10 \mathrm{~min}$ and then 5.6 mLethynyltrimethylsilane $(3.9 \mathrm{~g}, 40 \mathrm{mmol})$ was injected into the solution. The mixture was stirred overnight at r.t.. After removing the solvent under reduced pressure, the residue was purified by column chromatography (petroleum ether/acetone $=30 / 1$ ) to 
afford a yellow oil (4.1g, 80\%). ${ }^{1} \mathbf{H}$ NMR $\left(400 \mathrm{MHz}, \mathrm{CDCl}_{3}\right) \delta 7.73(\mathrm{~s}, 2 \mathrm{H}), 6.58(\mathrm{~d}, J=$ $7.2 \mathrm{~Hz}, 1 \mathrm{H}), 5.14(\mathrm{~s}, 2 \mathrm{H}), 4.74(\mathrm{~m}, 1 \mathrm{H}), 4.24-4.08(\mathrm{~m}, 2 \mathrm{H}), 1.72-1.56(\mathrm{~m}, 2 \mathrm{H}), 1.48(\mathrm{~d}$, $J=7.1 \mathrm{~Hz}, 3 \mathrm{H}), 1.38-1.20(\mathrm{~m}, 10 \mathrm{H}), 0.87(\mathrm{t}, J=6.8 \mathrm{~Hz}, 3 \mathrm{H}), 0.26(\mathrm{~s}, 18 \mathrm{H}) .{ }^{13} \mathbf{C} \mathbf{N M R}$ (101 MHz, $\left.\mathrm{CDCl}_{3}\right) \delta 173.46,165.14,151.94,131.68,122.33,106.88,101.14,100.10$, $65.70,48.46,31.73,30.90,29.12,28.50,25.78,22.60,18.82,14.06,-0.03$. HRMS (ESI): calcd for $\mathrm{C}_{28} \mathrm{H}_{45} \mathrm{~N}_{2} \mathrm{O}_{3} \mathrm{Si}_{2}[\mathrm{M}+\mathrm{H}]+513.2963$ found 513.2965.

(S)-Octyl-2-(4-methyl-3,5-diethynylbenzamido)propanoate (2g). A solution of $11 \mathrm{~g}$ (3.1g, $6.1 \mathrm{mmol}$ ) in $30 \mathrm{~mL}$ THF was added a solution of tetrabutylammonium fluoride (4.8g, $18.1 \mathrm{mmol}$ ) in $10 \mathrm{~mL}$ THF at r.t.. The mixture was stirred for 30min. After removing the solvent under reduced pressure, the residue was redissolved in $30 \mathrm{~mL}$ $\mathrm{CHCl}_{3}$ and washed with water and brine. Then the organic layer was dried over anhydrous $\mathrm{Na}_{2} \mathrm{SO}_{4}$ and the solvent was removed under reduced pressure. The crude product was purified by column chromatography (petroleum ether/acetone $=10 / 1$ ) to afford a white solid (1.8g, 80\%). ${ }^{1} \mathbf{H}$ NMR (400 MHz, $\left.\mathrm{CDCl}_{3}\right) \delta 7.80$ (s, 2H), 6.60 (d, $J=$ $7.1 \mathrm{~Hz}, 1 \mathrm{H}), 5.18(\mathrm{~s}, 2 \mathrm{H}), 4.74(\mathrm{~m}, 1 \mathrm{H}), 4.26-4.10(\mathrm{~m}, 2 \mathrm{H}), 3.44(\mathrm{~s}, 2 \mathrm{H}), 1.74-1.59(\mathrm{~m}$, 2H), $1.49(\mathrm{~d}, J=7.1 \mathrm{~Hz}, 3 \mathrm{H}), 1.40-1.21(\mathrm{~m}, 10 \mathrm{H}), 0.88(\mathrm{t}, J=6.9 \mathrm{~Hz}, 3 \mathrm{H}) .{ }^{13} \mathbf{C}$ NMR $\left(101 \mathrm{MHz}, \mathrm{CDCl}_{3}\right) \delta 173.41,165.00,152.44,132.42,122.48,105.85,83.61,79.04,65.75$, 48.51, 31.74, 29.14, 28.51, 25.80, 22.61, 18.79, 14.07. HRMS (ESI): calcd for $\mathrm{C}_{22} \mathrm{H}_{29} \mathrm{~N}_{2} \mathrm{O}_{3}[\mathrm{M}+\mathrm{H}]+369.2173$ found 369.2174 .

Compound 4i. To a $100 \mathrm{~mL}$ two-necked flask charged with $\mathbf{3 a}(557.2 \mathrm{mg}, 1.0 \mathrm{mmol}), \mathbf{2 g}$ (184.2 $\mathrm{mg}, 0.50 \mathrm{mmol}), \mathrm{Pd}_{2}(\mathrm{dba})_{3}(13.7 \mathrm{mg}, 0.015 \mathrm{mmol})$ and $\mathrm{CuI}(5.7 \mathrm{mg}, 0.030 \mathrm{mmol})$, $\mathrm{PPh}_{3}\left(39.8 \mathrm{mg}, 0.15 \mathrm{mmol}\right.$ ) under argon, $50 \mathrm{~mL}$ degassed $\mathrm{Et}_{3} \mathrm{~N}$ was added via syringe. The mixture was stirred at $75^{\circ} \mathrm{C}$ for $12 \mathrm{~h}$, and then removed the solvent under reduced pressure. The residue was purified by column chromatography $\left(\mathrm{CHCl}_{3} / \mathrm{THF}=60 / 1\right)$ to afford a light yellow solid (138.4 mg, 22.6\%). ${ }^{1} \mathbf{H}$ NMR (400 MHz, $\left.\mathrm{CDCl}_{3}\right) \delta 8.11(\mathrm{~s}, 2 \mathrm{H})$, $7.92(\mathrm{~s}, 2 \mathrm{H}), 7.87(\mathrm{~s}, 2 \mathrm{H}), 7.51$ (d, $J=7.5 \mathrm{~Hz}, 2 \mathrm{H}), 7.11(\mathrm{~d}, J=7.5 \mathrm{~Hz}, 1 \mathrm{H}), 5.37(\mathrm{~s}, 2 \mathrm{H})$, $4.87-4.76(\mathrm{~m}, 3 \mathrm{H}), 4.25-4.03(\mathrm{~m}, 6 \mathrm{H}), 1.71-1.58(\mathrm{~m}, 6 \mathrm{H}), 1.51(\mathrm{~m}, 9 \mathrm{H}), 1.27$ (m, 
$30 \mathrm{H}), 0.87(\mathrm{t}, J=6.8 \mathrm{~Hz}, 9 \mathrm{H}) .{ }^{13} \mathbf{C}$ NMR $\left(101 \mathrm{MHz}, \mathrm{CDCl}_{3}\right) \delta 173.95,173.60,165.19$, $164.50,151.39,142.38,136.29,135.38,132.40,129.40,124.85,122.36,106.22,93.73$, $92.92,86.73,65.94,65.83,48.76,31.75,29.15,28.51,25.80,22.62,18.52,18.17$, 14.08.MS (MALDI-TOF): calcd for $\mathrm{C}_{58} \mathrm{H}_{76} \mathrm{I}_{2} \mathrm{~N}_{4} \mathrm{O}_{9} \mathrm{Na}$ [M $\left.+\mathrm{Na}\right]+1249.3594$ found 1249.2244 .

Compound 5i. To a $50 \mathrm{~mL}$ two-necked flask charged with compound $4 \mathbf{i}$ ( $245.4 \mathrm{mg}, 0.20$ $\mathrm{mmol}), \mathrm{Pd}\left(\mathrm{PPh}_{3}\right)_{2} \mathrm{Cl}_{2}(4.3 \mathrm{mg}, 0.006 \mathrm{mmol})$ and $\mathrm{CuI}(2.3 \mathrm{mg}, 0.012 \mathrm{mmol})$ under argon, $15 \mathrm{~mL}$ THF and $8 \mathrm{~mL} \mathrm{Et}_{3} \mathrm{~N}$ were added via syringe. The solution was stirred for $10 \mathrm{~min}$ and then 0.11 mLethynyltrimethylsilane $(78.4 \mathrm{mg}, 0.8 \mathrm{mmol}$ ) was injected into the solution. The mixture was stirred overnight at r.t. After removing the solvent under reduced pressure, the residue was purified by column chromatography $\left(\mathrm{CHCl}_{3} / \mathrm{THF}=\right.$ 90/1) to afford a yellow oil (210.6 mg, 90\%). ${ }^{1} \mathbf{H}$ NMR (400 MHz, $\left.\mathrm{CDCl}_{3}\right) \delta 7.90(\mathrm{~s}, 2 \mathrm{H})$, $7.85(\mathrm{dd}, J=4.6,3.1 \mathrm{~Hz}, 4 \mathrm{H}), 7.70$ (s, 2H), 7.21 (d, $J=7.1 \mathrm{~Hz}, 2 \mathrm{H}), 6.98(\mathrm{~d}, J=7.1 \mathrm{~Hz}$, 1H), 5.29 (s, 2H), $4.86-4.75(\mathrm{~m}, 3 \mathrm{H}), 4.25-4.06(\mathrm{~m}, 6 \mathrm{H}), 1.64(\mathrm{~m}, 6 \mathrm{H}), 1.52(\mathrm{~m}, 9 \mathrm{H})$, $1.27(\mathrm{~m}, 30 \mathrm{H}), 0.87(\mathrm{t}, J=6.7 \mathrm{~Hz}, 9 \mathrm{H}), 0.26(\mathrm{~s}, 18 \mathrm{H}) .{ }^{13} \mathbf{C} \mathbf{~ N M R}\left(101 \mathrm{MHz}, \mathrm{CDCl}_{3}\right)$ $\delta 173.67,173.31,165.22,165.16,151.29,137.17,134.43,132.22,130.43,129.96,124.17$, 123.46, 106.47, 102.92, 96.50, 93.78, 85.95, 65.86, 65.74, 48.74, 48.51, 31.73, 29.13, $28.50,25.78,22.60,18.64,18.41,14.06,-0.20,-0.53$. MS (MALDI-TOF): calcd for $\mathrm{C}_{68} \mathrm{H}_{94} \mathrm{~N}_{4} \mathrm{O}_{9} \mathrm{Si}_{2} \mathrm{Na}[\mathrm{M}+\mathrm{Na}]+1189.6452$ found 1189.4949 .

Compound 6i. A solution of compound $\mathbf{5 i}(175.2 \mathrm{mg}, 0.15 \mathrm{mmol})$ in $10 \mathrm{~mL}$ THF was added a solution of tetrabutylammonium fluoride $(117.5 \mathrm{mg}, 0.45 \mathrm{mmol})$ in $3 \mathrm{~mL}$ THF at r.t.. The mixture was stirred for $30 \mathrm{~min}$. After removing the solvent under reduced pressure, the residue was redissolved in $15 \mathrm{~mL} \mathrm{CHCl}_{3}$ and washed with water and brine. Then the organic layer was dried over anhydrous $\mathrm{Na}_{2} \mathrm{SO}_{4}$ and the solvent was removed under reduced pressure. The crude product was purified by column chromatography $\left(\mathrm{CHCl}_{3} / \mathrm{THF}=40 / 1\right)$ to afford a light yellow solid $(142.1 \mathrm{mg}, 93 \%) .{ }^{1} \mathbf{H} \mathbf{~ N M R}(400 \mathrm{MHz}$, $\left.\mathrm{CDCl}_{3}\right) \delta 7.91(\mathrm{~s}, 2 \mathrm{H}), 7.87(\mathrm{~s}, 2 \mathrm{H}), 7.80(\mathrm{~s}, 2 \mathrm{H}), 7.68(\mathrm{~s}, 2 \mathrm{H}), 7.42(\mathrm{~d}, J=7.4 \mathrm{~Hz}, 2 \mathrm{H})$, 
$7.07(\mathrm{~d}, J=7.5 \mathrm{~Hz}, 1 \mathrm{H}), 5.34(\mathrm{~s}, 2 \mathrm{H}), 4.86-4.74(\mathrm{~m}, 3 \mathrm{H}), 4.23-4.04(\mathrm{~m}, 6 \mathrm{H}), 3.14(\mathrm{~s}$, 2H), $1.63(\mathrm{~m}, 6 \mathrm{H}), 1.51(\mathrm{~m}, 9 \mathrm{H}), 1.34-1.21(\mathrm{~m}, 30 \mathrm{H}), 0.86(\mathrm{t}, J=6.8 \mathrm{~Hz}, 9 \mathrm{H}) .{ }^{13} \mathbf{C}$ NMR (101 MHz, $\left.\mathrm{CDCl}_{3}\right) \delta 173.81,173.44,165.28,165.16,151.40,137.28,134.42$, $132.31,130.78,130.31,123.54,123.09,122.43,106.35,93.57,86.13,81.80,78.99,65.87$, $65.76,48.75,31.74,30.30,29.14,28.50,25.79,22.60,18.52,18.24,14.06 . \mathrm{MS}$ (MALDI-TOF): calcd for $\mathrm{C}_{62} \mathrm{H}_{78} \mathrm{~N}_{4} \mathrm{O}_{9} \mathrm{Na}[\mathrm{M}+\mathrm{Na}]+1045.5661$ found 1045.5385.

Compound 1j. To a $100 \mathrm{~mL}$ two-necked flask charged with $\mathrm{Pd}_{2}(\mathrm{dba})_{3}(22.6 \mathrm{mg}, 0.025$ $\mathrm{mmol})$ and $\mathrm{CuI}(9.4 \mathrm{mg}, 0.049 \mathrm{mmol}), \mathrm{PPh}_{3}(65.6 \mathrm{mg}, 0.25 \mathrm{mmol})$ under argon, $20 \mathrm{~mL}$ degassed $\mathrm{Et}_{3} \mathrm{~N}$ and $40 \mathrm{~mL}$ degassed DMF were added via syringe. The solution was stirred at $85^{\circ} \mathrm{C}$ for $10 \mathrm{~min}$. A solution of compound $4 \mathrm{a}(60.6 \mathrm{mg}, 0.050 \mathrm{mmol})$ and compound $\mathbf{6 i}(51.2 \mathrm{mg}, 0.050 \mathrm{mmol})$ in $10 \mathrm{~mL}$ DMF was slowly injected into the flask within $48 \mathrm{~h}$ by a syringe pump and then the solution was stirred for further $12 \mathrm{~h}$. The reaction mixture was concentrated in vacuo and purified by column chromatography $\left(\mathrm{CHCl}_{3} / \mathrm{THF} / \mathrm{AcOH}=25 / 1 / 0.5\right)$ to afford the crude product. The crude product was then washed with a few acetone and methanol to give the desired product (14.1 $\mathrm{mg}, 14 \%)$ as a light yellow solid. ${ }^{1} \mathbf{H}$ NMR $\left(400 \mathrm{MHz}\right.$, DMSO- $\left.d_{6}\right) \delta 9.07(\mathrm{t}, J=7.2 \mathrm{~Hz}, 5 \mathrm{H}), 8.63(\mathrm{~s}, 1 \mathrm{H})$, 8.35 (s, 2H), 8.17 (s, 6H), 8.13 (s, 4H), 8.07 (s, 1H), 8.01 (s, 2H), 7.96 (s, 2H), 6.58 (s, 2H), $4.54-4.45(\mathrm{~m}, 6 \mathrm{H}), 4.07(\mathrm{~m}, 12 \mathrm{H}), 1.63-1.51(\mathrm{~m}, 12 \mathrm{H}), 1.43(\mathrm{~m}, 18 \mathrm{H}), 1.21(\mathrm{~m}$, $60 \mathrm{H}), 0.80(\mathrm{~m}, 18 \mathrm{H}) .{ }^{13} \mathbf{C}$ NMR $\left(101 \mathrm{MHz}, \mathrm{DMF}-d_{7}\right) \delta 172.80,172.42,172.38,164.71$, $164.56,152.82,135.15,135.10,134.81,130.42,129.90,129.73,123.67,123.03,122.97$, $122.61,105.34,93.10,88.80,88.72,88.38,86.83,64.40,64.23,48.90,31.34,28.80$, 28.54, 28.26, 25.45, 22.15, 16.35, 16.22, 13.33. MS (MALDI-TOF): calcd for $\mathrm{C}_{120} \mathrm{H}_{151} \mathrm{~N}_{7} \mathrm{O}_{18} \mathrm{Na}[\mathrm{M}+\mathrm{Na}]+2001.1008$ found 2001.16.

Compound 1k. To a $100 \mathrm{~mL}$ two-necked flask charged with $\mathrm{Pd}_{2}(\mathrm{dba})_{3}(22.6 \mathrm{mg}, 0.025$ $\mathrm{mmol})$ and $\mathrm{CuI}(9.4 \mathrm{mg}, 0.049 \mathrm{mmol}), \mathrm{PPh}_{3}(65.6 \mathrm{mg}, 0.25 \mathrm{mmol})$ under argon, $20 \mathrm{~mL}$ degassed $\mathrm{Et}_{3} \mathrm{~N}$ and $40 \mathrm{~mL}$ degassed DMF were added via syringe. The solution was stirred at $85^{\circ} \mathrm{C}$ for $10 \mathrm{~min}$. A solution of compound $4 \mathbf{i}(61.4 \mathrm{mg}, 0.050 \mathrm{mmol})$ and 
compound 6i (51.2 mg, $0.050 \mathrm{mmol}$ ) in $10 \mathrm{~mL}$ DMF was slowly injected into the flask within $48 \mathrm{~h}$ by a syringe pump and then the solution was stirred for further $12 \mathrm{~h}$. The reaction mixture was concentrated in vacuo and purified by column chromatography $\left(\mathrm{CHCl}_{3} / \mathrm{THF} / \mathrm{AcOH}=25 / 1 / 0.5\right)$ to afford the crude product. The crude product was then washed with a few acetone and methanol to give the desired product (13.7 $\mathrm{mg}, 14 \%)$ as a light yellow solid. ${ }^{1} \mathbf{H}$ NMR (400 MHz, DMF- $\left.d_{7}\right) \delta 9.23(\mathrm{~d}, J=6.8 \mathrm{~Hz}, 4 \mathrm{H}), 8.83$ (d, $J=$ $6.8 \mathrm{~Hz}, 2 \mathrm{H}), 8.39$ (d, $J=1.3 \mathrm{~Hz}, 4 \mathrm{H}), 8.35-8.25(\mathrm{~m}, 8 \mathrm{H}), 8.22(\mathrm{~s}, 4 \mathrm{H}), 7.10(\mathrm{~s}, 4 \mathrm{H})$, $4.77-4.61(\mathrm{~m}, 6 \mathrm{H}), 4.28-4.12(\mathrm{~m}, 12 \mathrm{H}), 1.70(\mathrm{~m}, 12 \mathrm{H}), 1.61(\mathrm{~m}, 18 \mathrm{H}), 1.48-1.23(\mathrm{~m}$, $60 \mathrm{H}), 0.91(\mathrm{t}, J=6.6 \mathrm{~Hz}, 18 \mathrm{H}) .{ }^{13} \mathbf{C}$ NMR (101 MHz, DMF- $\left.d_{7}\right) \delta 173.13,172.74,165.07$, $164.99,153.33,137.32,135.29,133.02,130.20,130.08,124.05,123.02,105.71,93.33$, $89.06,87.26,64.72,64.55,49.20,31.67,29.35,29.09,28.88,28.58,25.78,22.48,16.69$, 16.55, 13.66. MS (MALDI-TOF): calcd for $\mathrm{C}_{120} \mathrm{H}_{152} \mathrm{~N}_{8} \mathrm{O}_{18} \mathrm{Na}[\mathrm{M}+\mathrm{Na}]+2016.1117$ found 2017.19 .

(S)-isopentyl-2-(4-fluoro-3,5-diiodobenzamido)propanoate (3e). To a $250 \mathrm{~mL}$ round bottom flask was added 4-fluoro-3,5-diiodobenzoic acid (10.0 g, $25.5 \mathrm{mmol}), 100 \mathrm{~mL}$ redistilled $\mathrm{CH}_{2} \mathrm{Cl}_{2}$ and $2.4 \mathrm{~mL}(\mathrm{COCl})_{2}$ (Oxalyl chloride) $(3.6 \mathrm{~g}, 28.3 \mathrm{mmol})$, then $0.1 \mathrm{~mL}$ DMF was added dropwise. The solution was stirred for 6hrs. After removing the solvent and the redundant $(\mathrm{COCl})_{2}$ under reduced pressure, the residue was resolve in $20 \mathrm{~mL}$ redistilled $\mathrm{CH}_{2} \mathrm{Cl}_{2}$ and added dropwise to a $250 \mathrm{~mL}$ ice-cooled round bottom flask containing $100 \mathrm{~mL}$ redistilled $\mathrm{CH}_{2} \mathrm{Cl}_{2}, 11 \mathrm{~mL} \mathrm{Et}_{3} \mathrm{~N}(8.0 \mathrm{~g}, 79.5 \mathrm{mmol})$ and (S)-isopentyl-2-aminopropanoate hydrochloride (5.3g, $27.2 \mathrm{mmol})$. The mixture was stirred for $8 \mathrm{~h}$ at r.t.. The solution was washed with water and brine, and then concentrated in vacuo. The crude product was purified by column chromatography (petroleum ether/acetone $=5 / 1)$ to afford a white solid $(11.4 \mathrm{~g}, 84 \%) .{ }^{\mathbf{1}} \mathbf{H} \mathbf{~ N M R}\left(400 \mathrm{MHz}, \mathrm{CDCl}_{3}\right)$ $\delta 8.19(\mathrm{~d}, J=5.4 \mathrm{~Hz}, 2 \mathrm{H}), 6.71(\mathrm{~d}, J=6.8 \mathrm{~Hz}, 1 \mathrm{H}), 4.77(\mathrm{p}, J=7.1 \mathrm{~Hz}, 1 \mathrm{H}), 4.34-4.21$ (m, 2H), $1.75(\mathrm{~m}, 1 \mathrm{H}), 1.62(\mathrm{~m}, 3 \mathrm{H}), 1.56(\mathrm{~d}, J=7.1 \mathrm{~Hz}, 3 \mathrm{H}), 0.99(\mathrm{~d}, J=6.6 \mathrm{~Hz}, 6 \mathrm{H})$. ${ }^{13} \mathbf{C}$ NMR (101 MHz, $\left.\mathrm{CDCl}_{3}\right) \delta 172.94,162.73,161.98(\mathrm{~d}, J=247.8 \mathrm{~Hz}), 138.36(\mathrm{~d}, J=$ 
$2.2 \mathrm{~Hz}), 132.68(\mathrm{~d}, J=4.1 \mathrm{~Hz}), 80.14(\mathrm{~d}, J=29.7 \mathrm{~Hz}), 64.25,48.49,36.85,24.72,22.15$, 22.08, 18.11. HRMS (ESI): calcd for $\mathrm{C}_{15} \mathrm{H}_{19} \mathrm{FI}_{2} \mathrm{NO}_{3}[\mathrm{M}+\mathrm{H}]+533.9433$ found 533.9436 .

\section{(S)-isopentyl-2-(4-fluoro-3,5-bis(2-(trimethylsilyl)ethynyl)benzamido)propanoate}

(11e). To a $100 \mathrm{~mL}$ two-necked flask charged with $3 \mathrm{e}$ (5.3g, $10.0 \mathrm{mmol}), \mathrm{Pd}\left(\mathrm{PPh}_{3}\right)_{2} \mathrm{Cl}_{2}$ (214.5 mg, $0.3 \mathrm{mmol})$ and $\mathrm{CuI}(114.3 \mathrm{mg}, 0.6 \mathrm{mmol})$ under argon, $40 \mathrm{~mL}$ THF and 20 $\mathrm{mL} \mathrm{Et}{ }_{3} \mathrm{~N}$ were added via syringe. The solution was stirred for $10 \mathrm{~min}$ and then 5.6 mLethynyltrimethylsilane (3.9g, $40 \mathrm{mmol})$ was injected into the solution. The mixture was stirred overnight at r.t.. After removing the solvent under reduced pressure, the residue was purified by column chromatography (petroleum ether/acetone $=25 / 1$ ) to afford a yellow oil (3.5g, 74.0\%). ${ }^{1} \mathbf{H}$ NMR $\left(400 \mathrm{MHz}, \mathrm{CDCl}_{3}\right) \delta 7.83(\mathrm{~d}, J=6.1 \mathrm{~Hz}, 2 \mathrm{H})$, $6.76(\mathrm{~d}, J=7.0 \mathrm{~Hz}, 1 \mathrm{H}), 4.73(\mathrm{~m}, 1 \mathrm{H}), 4.29-4.14(\mathrm{~m}, 2 \mathrm{H}), 1.76-1.65(\mathrm{~m}, 1 \mathrm{H}), 1.57(\mathrm{~m}$, $2 \mathrm{H}), 1.50(\mathrm{~d}, J=7.1 \mathrm{~Hz}, 3 \mathrm{H}), 0.94(\mathrm{~d}, J=6.6 \mathrm{~Hz}, 6 \mathrm{H}), 0.27$ (s, $18 \mathrm{H}) .{ }^{13} \mathbf{C}$ NMR $(101$ $\mathrm{MHz}, \mathrm{CDCl} 3) \delta 172.77,164.53(\mathrm{~d}, \mathrm{~J}=263.0 \mathrm{~Hz}), 164.02,132.17$ (d, J = 1.9 Hz), 129.53 (d, J = 4.2 Hz), 112.36 (d, J = 16.7 Hz), 101.87 (d, J = 3.6 Hz), 95.77, 64.10, 48.40, 36.85, 24.70, 22.09, 22.03, 18.26, -0.65. HRMS (ESI): calcd for $\mathrm{C}_{25} \mathrm{H}_{37} \mathrm{FNO}_{3} \mathrm{Si}_{2}[\mathrm{M}+\mathrm{H}]^{+}$ 474.2291 found 474.2295 .

(S)-isopentyl-2-(3,5-diethynyl-4-fluorobenzamido)propanoate (2e). A solution of 11e (2.8g, $5.9 \mathrm{mmol})$ in $30 \mathrm{~mL}$ THF was added a solution of tetrabutylammonium fluoride (4.6g, $17.7 \mathrm{mmol})$ in $10 \mathrm{~mL}$ THF at r.t. The mixture was stirred for 30min. After removing the solvent under reduced pressure, the residue was redissolved in $30 \mathrm{~mL}$ $\mathrm{CHCl}_{3}$ and washed with water and brine. Then the organic layer was dried over anhydrous $\mathrm{Na}_{2} \mathrm{SO}_{4}$ and the solvent was removed under reduced pressure. The crude product was purified by column chromatography (petroleum ether/acetone $=8 / 1$ ) to afford a white solid (1.4g, 72\%). ${ }^{1} \mathbf{H}$ NMR $\left(400 \mathrm{MHz}, \mathrm{CDCl}_{3}\right) \delta 7.92(\mathrm{~d}, J=6.1 \mathrm{~Hz}, 2 \mathrm{H})$, $6.73(\mathrm{~s}, 1 \mathrm{H}), 4.76(\mathrm{~m}, 1 \mathrm{H}), 4.30-4.16(\mathrm{~m}, 2 \mathrm{H}), 3.40(\mathrm{~s}, 2 \mathrm{H}), 1.72(\mathrm{~m}, 1 \mathrm{H}), 1.59(\mathrm{~m}, 2 \mathrm{H})$, $1.53(\mathrm{~d}, J=7.1 \mathrm{~Hz}, 3 \mathrm{H}), 0.96(\mathrm{~d}, J=6.6 \mathrm{~Hz}, 6 \mathrm{H}) .{ }^{13} \mathbf{C} \mathbf{N M R}(101 \mathrm{MHz}, \mathrm{CDCl} 3) \delta 172.74$, 165.09 (d, J = 266.1 Hz), 163.77, 132.87 (d, J = 2.3 Hz), 129.87, 111.47 (d, J = 16.4 Hz), 
83.87 (d, J = 3.6 Hz), 74.99, 64.20, 48.46, 36.86, 24.72, 22.10, 22.04, 18.29. HRMS (ESI): calcd for $\mathrm{C}_{19} \mathrm{H}_{21} \mathrm{FNO}_{3}[\mathrm{M}+\mathrm{H}]^{+} 330.1500$ found 330.1500 .

Compound4e. To a $100 \mathrm{~mL}$ two-necked flask charged with $\mathbf{3 e}(532.9 \mathrm{mg}, 1.0 \mathrm{mmol}), \mathbf{2 e}$ (164.6 mg, $0.50 \mathrm{mmol}), \mathrm{Pd}_{2}(\mathrm{dba})_{3}(13.7 \mathrm{mg}, 0.015 \mathrm{mmol})$ and $\mathrm{CuI}(5.7 \mathrm{mg}, 0.030 \mathrm{mmol})$, $\mathrm{PPh}_{3}\left(39.8 \mathrm{mg}, 0.15 \mathrm{mmol}\right.$ ) under argon, $50 \mathrm{~mL}$ degassed $\mathrm{Et}_{3} \mathrm{~N}$ was added via syringe. The mixture was stirred at $75^{\circ} \mathrm{C}$ for $12 \mathrm{hrs}$, and then removed the solvent under reduced pressure. The residue was purified by column chromatography $\left(\mathrm{CHCl}_{3} / \mathrm{THF}=40 / 1\right)$ to afford a light yellow solid (124.2 mg, 22\%). ${ }^{1} \mathbf{H}$ NMR $\left(400 \mathrm{MHz}, \mathrm{CDCl}_{3}\right) \delta 8.25(\mathrm{~d}, J=$ $4.2 \mathrm{~Hz}, 2 \mathrm{H}), 8.01(\mathrm{~d}, J=6.0 \mathrm{~Hz}, 2 \mathrm{H}), 7.95(\mathrm{~d}, J=4.8 \mathrm{~Hz}, 2 \mathrm{H}), 7.07$ (d, $J=7.2 \mathrm{~Hz}, 1 \mathrm{H})$, $6.97(\mathrm{~d}, J=7.3 \mathrm{~Hz}, 2 \mathrm{H}), 4.81(\mathrm{~m}, 3 \mathrm{H}), 4.25(\mathrm{~m}, 6 \mathrm{H}), 1.73(\mathrm{~m}, 3 \mathrm{H}), 1.66-1.48(\mathrm{~m}, 18 \mathrm{H})$, $0.96(\mathrm{~d}, J=6.6 \mathrm{~Hz}, 18 \mathrm{H}) .{ }^{13} \mathbf{C}$ NMR $\left(101 \mathrm{MHz}, \mathrm{DMSO}-d_{6}\right) \delta 172.47,163.26,162.88(\mathrm{~d}, J$ $=212.6 \mathrm{~Hz}), 162.48(\mathrm{~d}, J=210.9 \mathrm{~Hz}), 139.84,133.77,132.93,131.76,130.87,110.87(\mathrm{~d}$, $J=15.9 \mathrm{~Hz}), 110.12(\mathrm{~d}, J=19.1 \mathrm{~Hz}), 87.70,87.15,83.37,83.10,63.10(\mathrm{~d}, J=2.9 \mathrm{~Hz})$, 48.82, 36.95, 24.61, 22.40, 22.32, 16.72. MS (MALDI-TOF): calcd for $\mathrm{C}_{49} \mathrm{H}_{54} \mathrm{~F}_{3} \mathrm{I}_{2} \mathrm{~N}_{3} \mathrm{O}_{9} \mathrm{Na}[\mathrm{M}+\mathrm{Na}]^{+} 1162.1794$ found 1162.1914 .

Compound5e. To a $50 \mathrm{~mL}$ two-necked flask charged with compound $4 \mathbf{e}(227.8 \mathrm{mg}, 0.20$ $\mathrm{mmol}), \mathrm{Pd}\left(\mathrm{PPh}_{3}\right)_{2} \mathrm{Cl}_{2}(4.3 \mathrm{mg}, 0.006 \mathrm{mmol})$ and $\mathrm{CuI}(2.3 \mathrm{mg}, 0.012 \mathrm{mmol})$ under argon, $15 \mathrm{~mL}$ THF and $8 \mathrm{~mL} \mathrm{Et}_{3} \mathrm{~N}$ were added via syringe. The solution was stirred for $10 \mathrm{~min}$ and then 0.11 mLethynyltrimethylsilane $(78.4 \mathrm{mg}, 0.8 \mathrm{mmol}$ ) was injected into the solution. The mixture was stirred overnight at r.t.. After removing the solvent under reduced pressure, the residue was purified by column chromatography $\left(\mathrm{CHCl}_{3} / \mathrm{THF}=\right.$ 60/1) to afford a yellow oil (174.9 mg, 81\%). ${ }^{1} \mathbf{H}$ NMR (400 MHz, $\left.\mathrm{CDCl}_{3}\right) \delta 8.05(\mathrm{~d}, J=$ $5.9 \mathrm{~Hz}, 2 \mathrm{H}), 7.97$ (dd, $J=11.8,5.8 \mathrm{~Hz}, 4 \mathrm{H}), 7.12(\mathrm{~d}, J=6.1 \mathrm{~Hz}, 1 \mathrm{H}), 6.99$ (d, $J=7.0 \mathrm{~Hz}$, 2H), $4.83(\mathrm{~m}, 13 \mathrm{H}), 4.26(\mathrm{~m}, 6 \mathrm{H}), 1.74(\mathrm{~m}, 3 \mathrm{H}), 1.60$ (m, 18H), 0.97 (d, $J=6.6 \mathrm{~Hz}, 18 \mathrm{H})$, $0.32(\mathrm{~s}, 18 \mathrm{H}) .{ }^{13} \mathrm{C}$ NMR $\left(101 \mathrm{MHz}, \mathrm{CDCl}_{3}\right) \delta 172.82,164.21$ (d, $\left.J=264.0 \mathrm{~Hz}\right), 163.98$, $163.88,163.80$ (d, $J=262.6 \mathrm{~Hz}), 132.82$ (d, $J=45.6 \mathrm{~Hz}), 131.86,130.10,129.82,112.65$ (d, $J=16.3 \mathrm{~Hz}), 111.83$ (d, $J=16.1 \mathrm{~Hz}), 111.49$ (d, $J=16.5 \mathrm{~Hz}), 102.31,95.58,87.45$, 
86.96, 64.14, 48.49 (d, $J=3.3 \mathrm{~Hz}), 36.87,24.72,22.10,22.05,18.21,18.17,-0.63 . \mathrm{MS}$

(MALDI-TOF): calcd for $\mathrm{C}_{59} \mathrm{H}_{72} \mathrm{~F}_{3} \mathrm{~N}_{3} \mathrm{O}_{9} \mathrm{Si}_{2} \mathrm{Na}[\mathrm{M}+\mathrm{Na}]^{+} 1102.4651$ found 1102.3342 .

Compound 6e. A solution of compound 5e (161.9 mg, $0.15 \mathrm{mmol})$ in $10 \mathrm{~mL}$ THF was added a solution of tetrabutylammonium fluoride $(117.5 \mathrm{mg}, 0.45 \mathrm{mmol})$ in $3 \mathrm{~mL}$ THF at r.t.. The mixture was stirred for 30min. After removing the solvent under reduced pressure, the residue was redissolved in $15 \mathrm{~mL} \mathrm{CHCl}_{3}$ and washed with water and brine. Then the organic layer was dried over anhydrous $\mathrm{Na}_{2} \mathrm{SO}_{4}$ and the solvent was removed under reduced pressure. The crude product was purified by column chromatography $\left(\mathrm{CHCl}_{3} / \mathrm{THF}=25 / 1\right)$ to afford a light yellow solid $(98.5 \mathrm{mg}, 70 \%) .{ }^{1} \mathbf{H} \mathbf{~ N M R}(400 \mathrm{MHz}$, $\left.\mathrm{CDCl}_{3}\right) \delta 8.11-7.93(\mathrm{~m}, 6 \mathrm{H}), 6.90(\mathrm{~d}, J=7.1 \mathrm{~Hz}, 1 \mathrm{H}), 6.84(\mathrm{~d}, J=7.3 \mathrm{~Hz}, 2 \mathrm{H}), 4.85-$ $4.77(\mathrm{~m}, 3 \mathrm{H}), 4.28(\mathrm{~m}, 6 \mathrm{H}), 3.44(\mathrm{~s}, 2 \mathrm{H}), 1.75(\mathrm{~m}, 3 \mathrm{H}), 1.65-1.56(\mathrm{~m}, 18 \mathrm{H}), 0.98(\mathrm{~d}, J=$ $6.6 \mathrm{~Hz}, 18 \mathrm{H}) .{ }^{13} \mathrm{C}$ NMR $\left(101 \mathrm{MHz}, \mathrm{DMSO}-d_{6}\right) \delta 172.46,163.75(\mathrm{~d}, J=260.7 \mathrm{~Hz})$, $163.58,163.54,161.87,134.02(\mathrm{~d}, J=41.6 \mathrm{~Hz}), 133.33,130.99,130.73,111.11(\mathrm{~d}, J=$ $15.8 \mathrm{~Hz}), 110.85(\mathrm{~d}, J=15.7 \mathrm{~Hz}), 110.64$ (d, $J=15.8 \mathrm{~Hz}), 88.10,87.35$ (d, $J=30.2 \mathrm{~Hz})$, 75.32, 63.09, 48.81, 36.95, 24.62, 22.39, 22.31, 16.71. MS (MALDI-TOF): calcd for $\mathrm{C}_{53} \mathrm{H}_{56} \mathrm{~F}_{3} \mathrm{~N}_{3} \mathrm{O}_{9} \mathrm{Na}[\mathrm{M}+\mathrm{Na}]^{+} 958.3861$ found 958.3265 .

Macrocycle1e. To a $100 \mathrm{~mL}$ two-necked flask charged with $\operatorname{Pd}_{2}(\mathrm{dba})_{3}(22.6 \mathrm{mg}, 0.025$ $\mathrm{mmol})$ and $\mathrm{CuI}(9.4 \mathrm{mg}, 0.049 \mathrm{mmol}), \mathrm{PPh}_{3}(65.6 \mathrm{mg}, 0.25 \mathrm{mmol})$ under argon, $20 \mathrm{~mL}$ degassed $\mathrm{Et}_{3} \mathrm{~N}$ and $40 \mathrm{~mL}$ degassed $\mathrm{DMF}$ were added via syringe. The solution was stirred at $85^{\circ} \mathrm{C}$ for $10 \mathrm{~min}$. A solution of compound $4 \mathrm{e}(57.0 \mathrm{mg}, 0.050 \mathrm{mmol})$ and compound 6e $(46.8 \mathrm{mg}, 0.050 \mathrm{mmol})$ in $10 \mathrm{~mL}$ DMF was slowly injected into the flask within $48 \mathrm{~h}$ by a syringe pump and then the solution was stirred for further $12 \mathrm{~h}$. The reaction mixture was concentrated in vacuo and purified by column chromatography $\left(\mathrm{CHCl}_{3} / \mathrm{THF}=10 / 1\right.$ and then THF $)$ to afford the crude product. The crude product was then washed with a few acetone and methanol to give the desired product $(7.0 \mathrm{mg}, 8 \%)$ as a yellow solid. ${ }^{1}$ H NMR (400 MHz, DMF- $\left.d_{7}\right) \delta 9.23(\mathrm{~d}, J=6.2 \mathrm{~Hz}, 6 \mathrm{H}), 8.36(\mathrm{~d}, J=6.1$ $\mathrm{Hz}, 12 \mathrm{H}), 4.86-4.77(\mathrm{~m}, 6 \mathrm{H}), 4.39(\mathrm{~m}, 12 \mathrm{H}), 1.91(\mathrm{~m}, 6 \mathrm{H}), 1.85-1.50(\mathrm{~m}, 36 \mathrm{H}), 1.11$ 
$(\mathrm{d}, J=6.6 \mathrm{~Hz}, 36 \mathrm{H}) .{ }^{13} \mathbf{C}$ NMR $\left(101 \mathrm{MHz}, \mathrm{DMF}-d_{7}\right) \delta 172.64,163.76,162.15,133.44$, 130.44, 111.17 (d, $J=15.8 \mathrm{~Hz}), 87.37,63.13,49.23,37.11,24.81,21.92,21.86,16.41$. MS (MALDI-TOF): calcd for $\mathrm{C}_{102} \mathrm{H}_{108} \mathrm{~F}_{6} \mathrm{~N}_{6} \mathrm{O}_{18} \mathrm{Na}[\mathrm{M}+\mathrm{Na}]^{+} 1841.7517$ found 1841.6089 .

Macrocycle1l. To a $100 \mathrm{~mL}$ two-necked flask charged with $\mathrm{Pd}_{2}(\mathrm{dba})_{3}(22.6 \mathrm{mg}, 0.025$ $\mathrm{mmol})$ and $\mathrm{CuI}$ (9.4 mg, $0.049 \mathrm{mmol}), \mathrm{PPh}_{3}(65.6 \mathrm{mg}, 0.25 \mathrm{mmol})$ under argon, $20 \mathrm{~mL}$ degassed $\mathrm{Et}_{3} \mathrm{~N}$ and $40 \mathrm{~mL}$ degassed DMF were added via syringe. The solution was stirred at $85^{\circ} \mathrm{C}$ for $10 \mathrm{~min}$. A solution of compound $4 \mathbf{i}(61.3 \mathrm{mg}, 0.050 \mathrm{mmol})$ and compound $\mathbf{6 f}(51.3 \mathrm{mg}, 0.050 \mathrm{mmol})$ in $10 \mathrm{~mL}$ DMF was slowly injected into the flask within $48 \mathrm{~h}$ by a syringe pump and then the solution was stirred for further $12 \mathrm{~h}$. The reaction mixture was concentrated in vacuo and purified by column chromatography $\left(\mathrm{CHCl}_{3} / \mathrm{THF} / \mathrm{AcOH}=25 / 1 / 0.5\right)$ to afford the crude product. The crude product was then washed with a few acetone and methanol to give the desired product $(12.1 \mathrm{mg}, 12 \%)$ as a light yellow solid. ${ }^{1} \mathbf{H}$ NMR (400 MHz, DMF- $\left.d_{7}\right) \delta 9.17(\mathrm{dd}, J=13.5,6.7 \mathrm{~Hz}, 5 \mathrm{H}), 8.76$ (d, $J=6.3 \mathrm{~Hz}, 1 \mathrm{H}), 8.41$ (s, 2H), 8.34 (s, 2H), 8.33 (s, 2H), 8.27 (m, 4H), 8.20 (s, 2H), 8.17 (s, 2H), $8.08(\mathrm{~s}, 2 \mathrm{H}), 7.88(\mathrm{~s}, 2 \mathrm{H}), 6.82(\mathrm{~s}, 2 \mathrm{H}), 4.72(\mathrm{~m}, 6 \mathrm{H}), 4.28-4.13(\mathrm{~m}, 12 \mathrm{H}), 1.79$ $-1.67(\mathrm{~m}, 12 \mathrm{H}), 1.63(\mathrm{~m}, 18 \mathrm{H}), 1.47-1.25(\mathrm{~m}, 60 \mathrm{H}), 0.91(\mathrm{t}, J=6.2 \mathrm{~Hz}, 18 \mathrm{H}) .{ }^{13} \mathbf{C}$ NMR (101 MHz, DMF- $\left.d_{7}\right) \delta 173.01,172.63,172.57,172.55,165.09,164.94,164.71$, 164.16, 154.35, 137.43, 137.00, 135.31, 134.84, 133.34, 132.96, 131.10, 130.79, 130.14, $130.00,129.98,123.86,123.34,122.76,122.70,111.89,111.38,105.49,94.08,93.35$, $89.50,88.39,86.97,82.42,64.59,49.13,31.53,28.99,28.96,28.45,25.65,22.34,16.40$, 13.51. MS (MALDI-TOF): calcd for $\mathrm{C}_{120} \mathrm{H}_{150} \mathrm{FN}_{7} \mathrm{O}_{18} \mathrm{Na}[\mathrm{M}+\mathrm{Na}]^{+} 2019.0914$ found 2018.9128. 


\section{III. ${ }^{1} \mathrm{H}$ NMR and ${ }^{13} \mathrm{C}$ NMR Spectra of Macrocycles 1a-I}

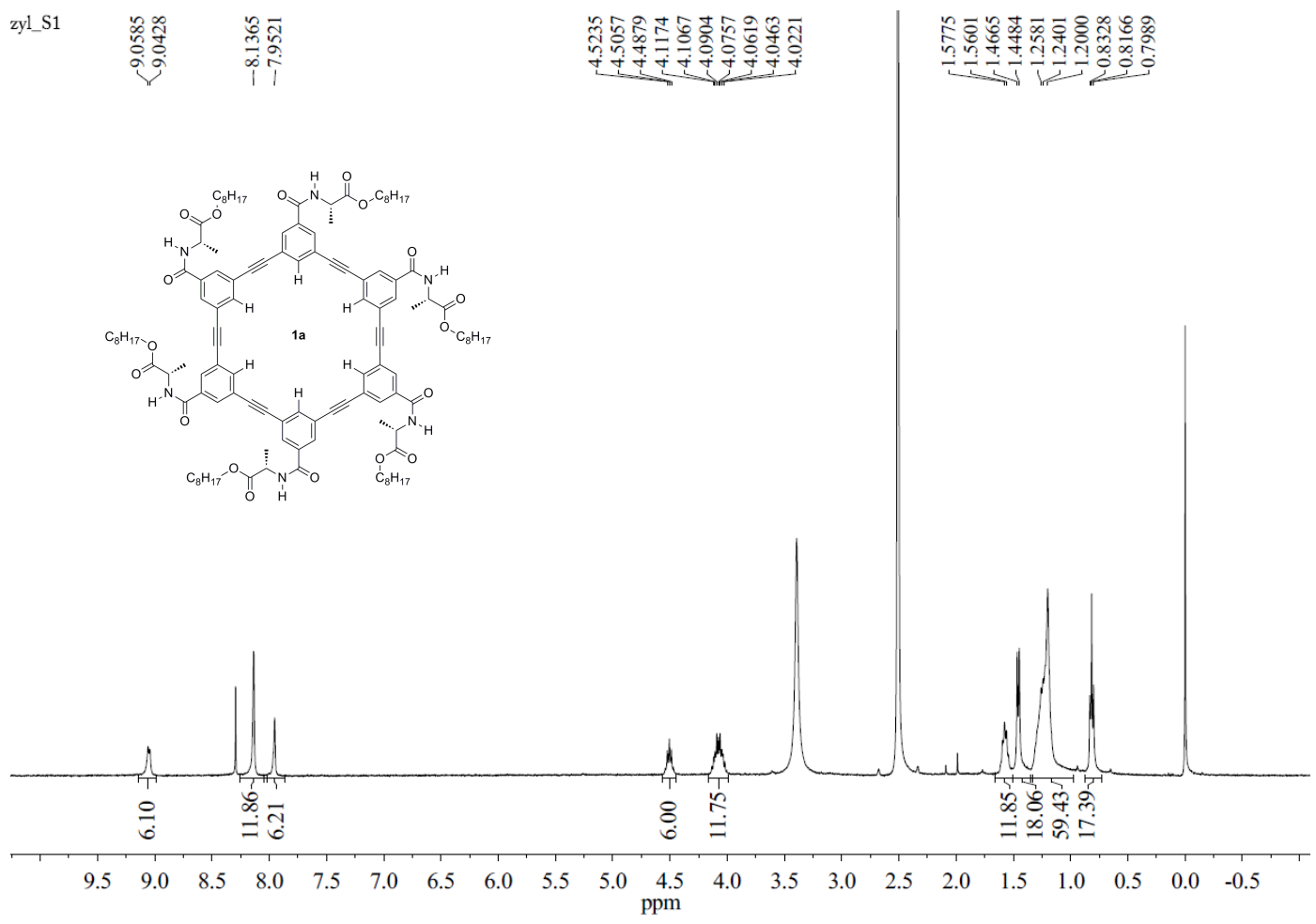

Figure S1 ${ }^{1} \mathrm{H}$ NMR Spectra of macrocycle $1 \mathrm{a}\left(2 \mathrm{mM}, 10 \% \mathrm{CDCl}_{3}+\mathrm{DMSO}-d_{6}\right)$
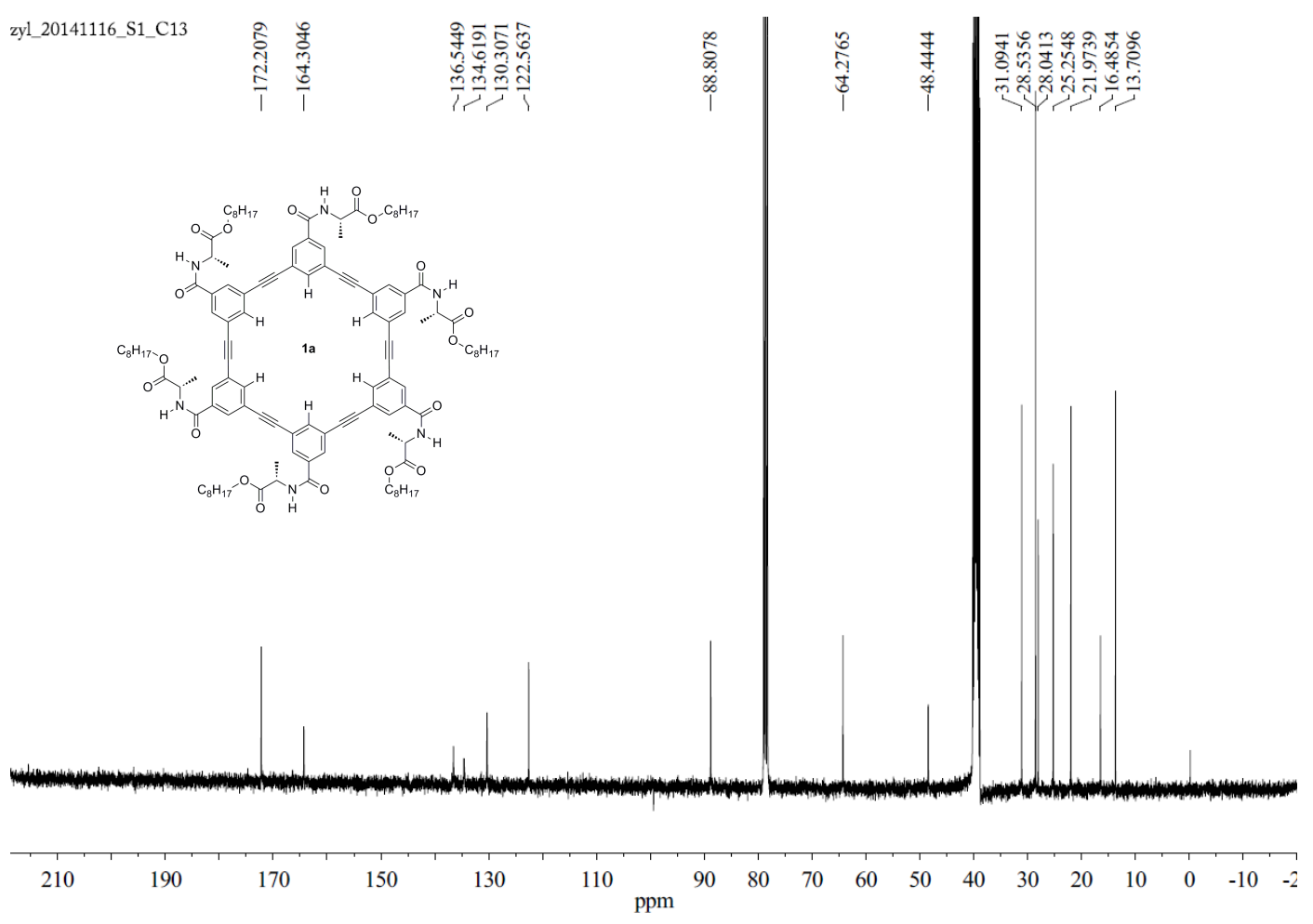

Figure S2 ${ }^{13} \mathrm{C}$ NMR Spectra of macrocycle 1a (4 mM, 20\%CDCl $\left.3+\mathrm{DMSO}-d_{6}\right)$ 


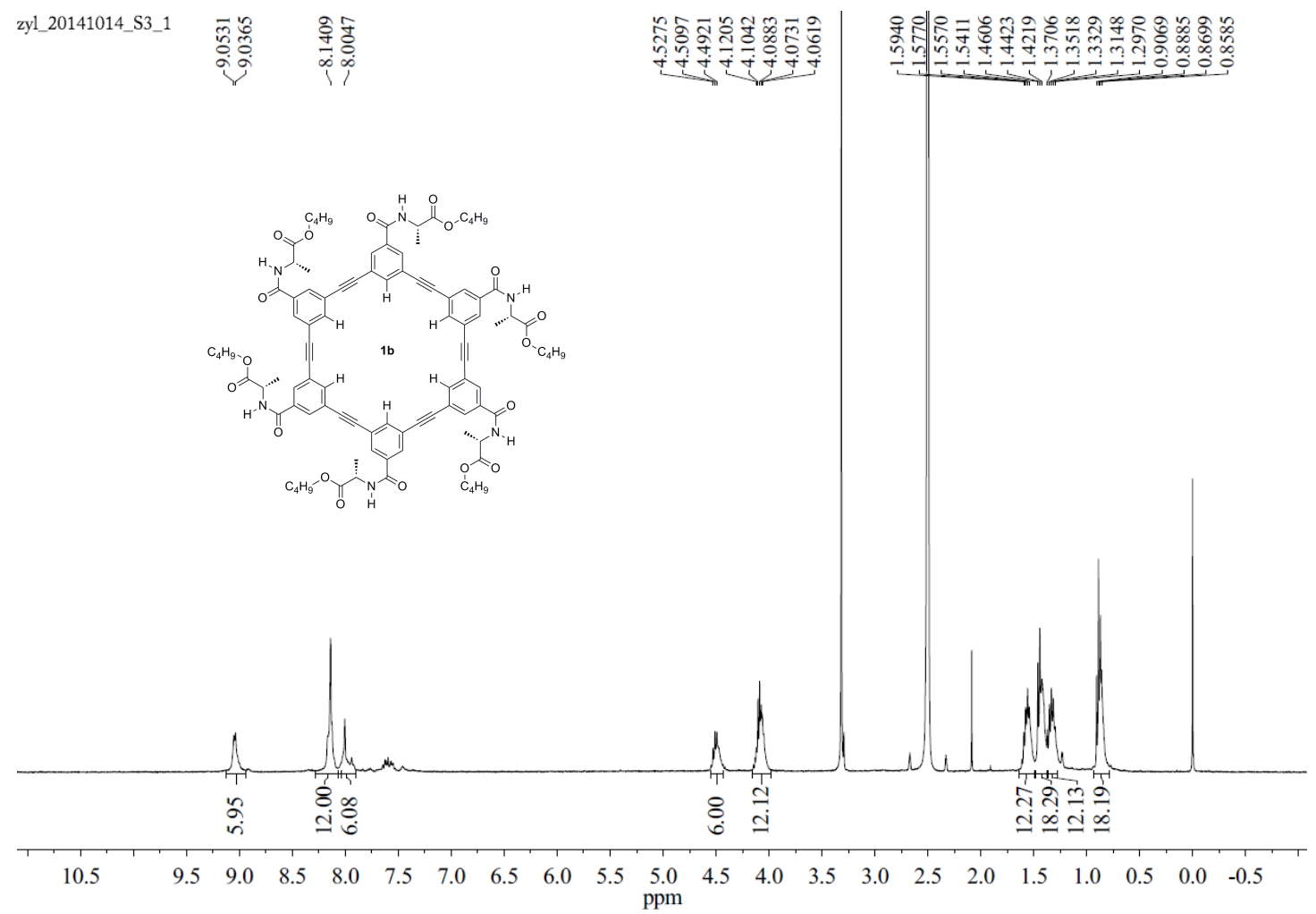

Figure $\mathbf{S 3}{ }^{1} \mathrm{H}$ NMR Spectra of macrocycle $\mathbf{1 b}\left(2 \mathrm{mM}\right.$, DMSO- $\left.d_{6}\right)$

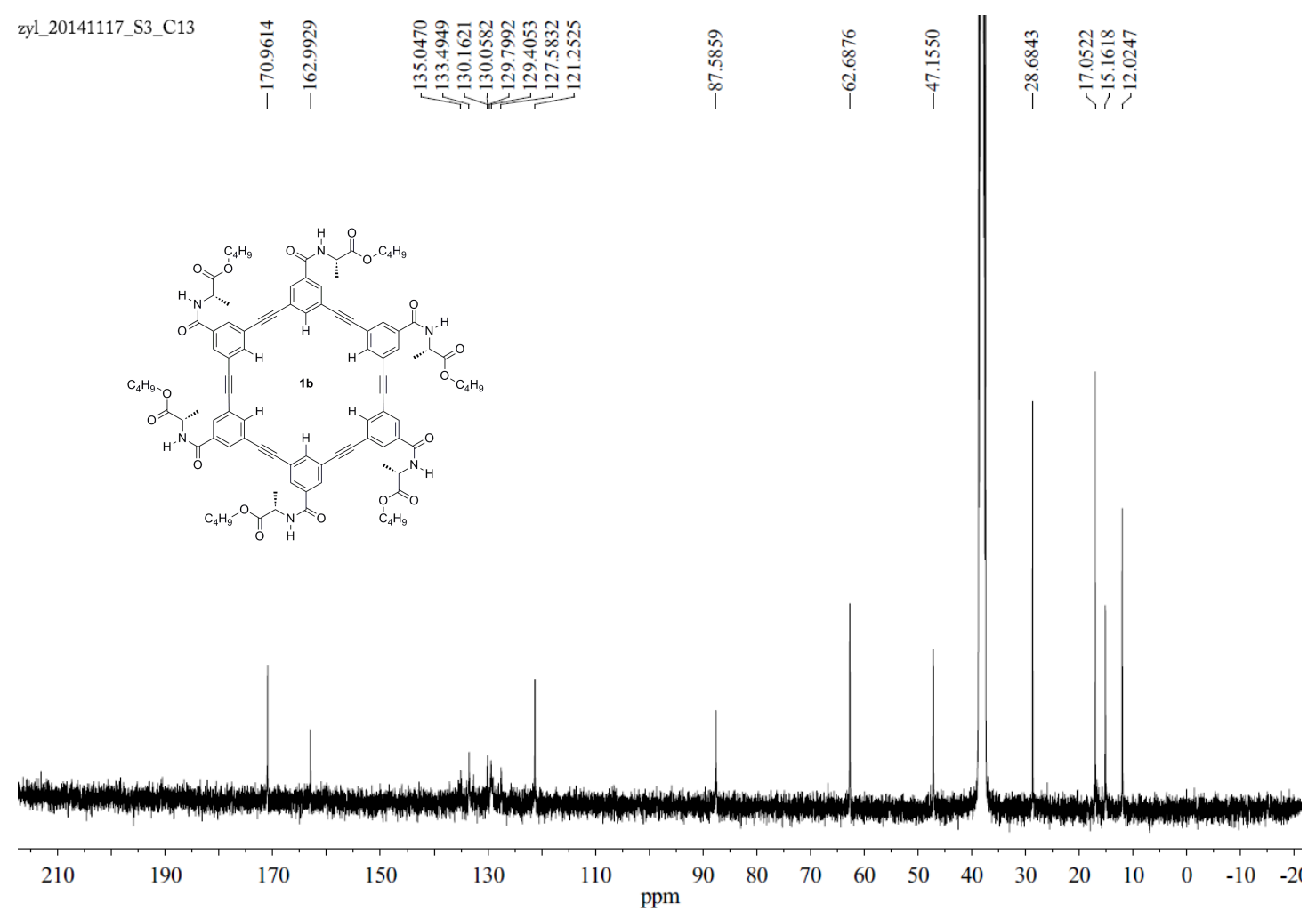

Figure S4 ${ }^{13} \mathrm{C}$ NMR Spectra of macrocycle $1 \mathrm{~b}$ (4 mM, DMSO- $\left.d_{6}\right)$ 


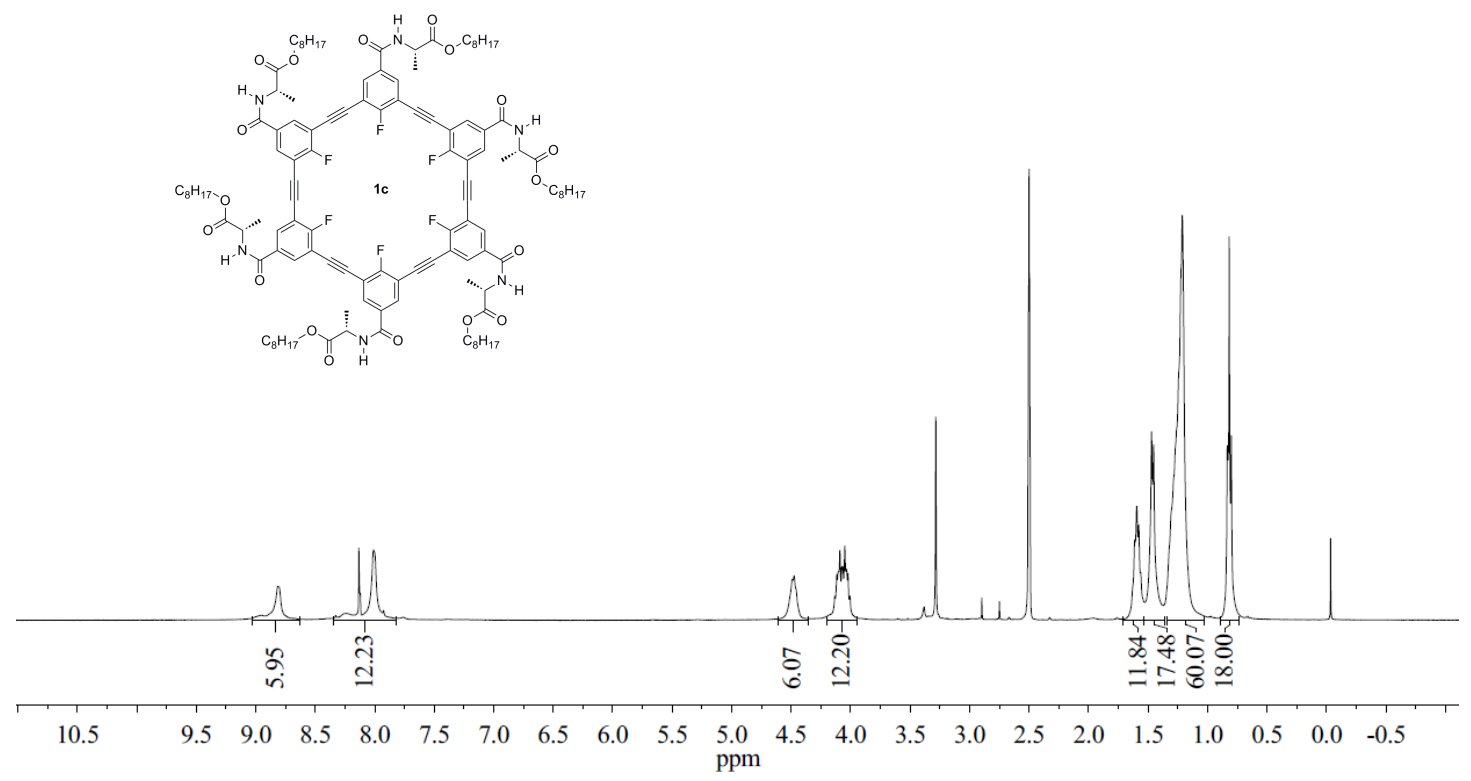

Figure S5 ${ }^{1} \mathrm{H}$ NMR Spectra of macrocycle $1 \mathrm{c}\left(2 \mathrm{mM}, 10 \% \mathrm{CDCl}_{3}+\mathrm{DMSO}-d_{6}\right)$

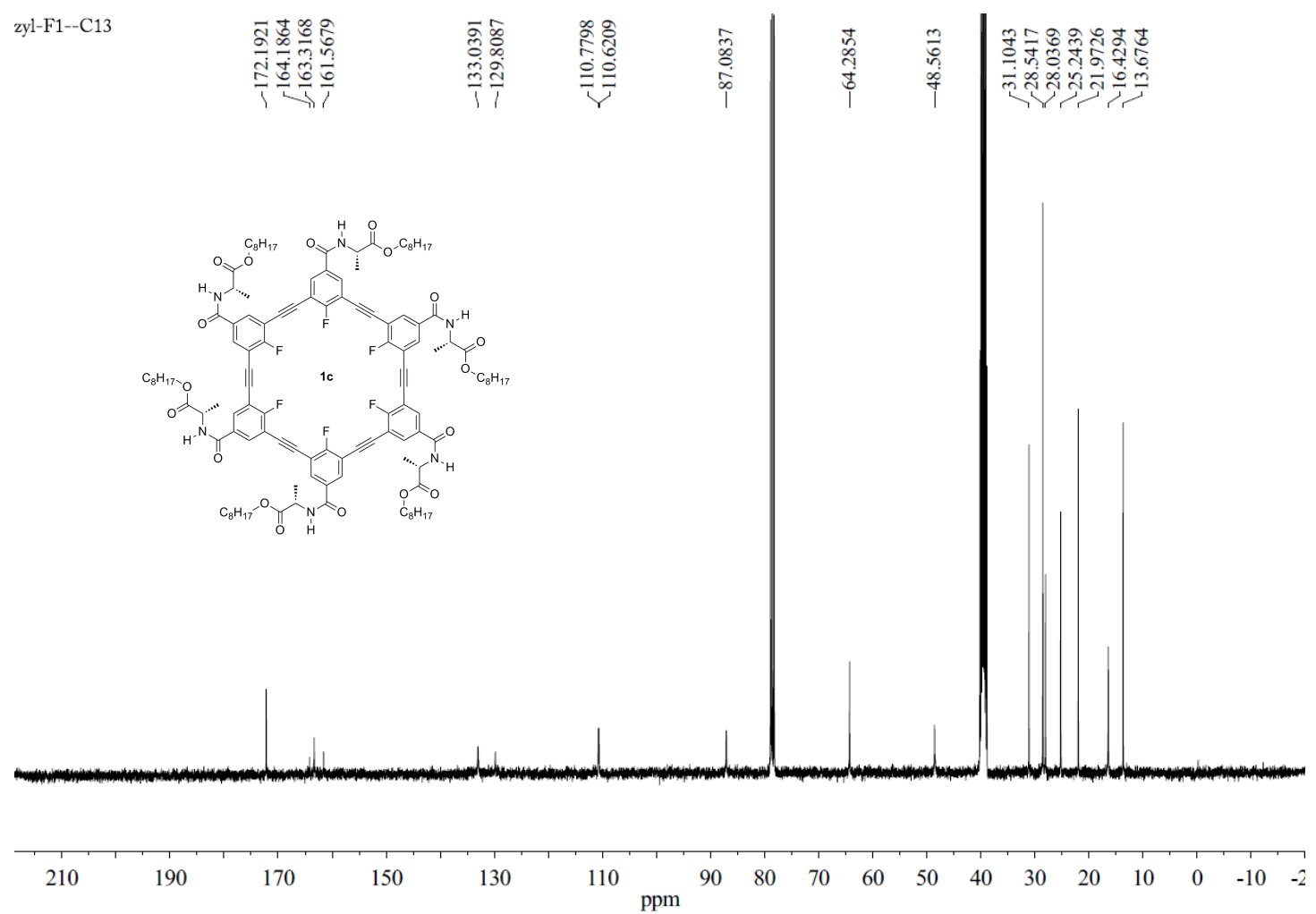

Figure S6 ${ }^{13} \mathrm{C}$ NMR Spectra of macrocycle 1c (4 mM, 20\% $\mathrm{CDCl}_{3}$ in DMSO- $\left.d_{6}\right)$ 


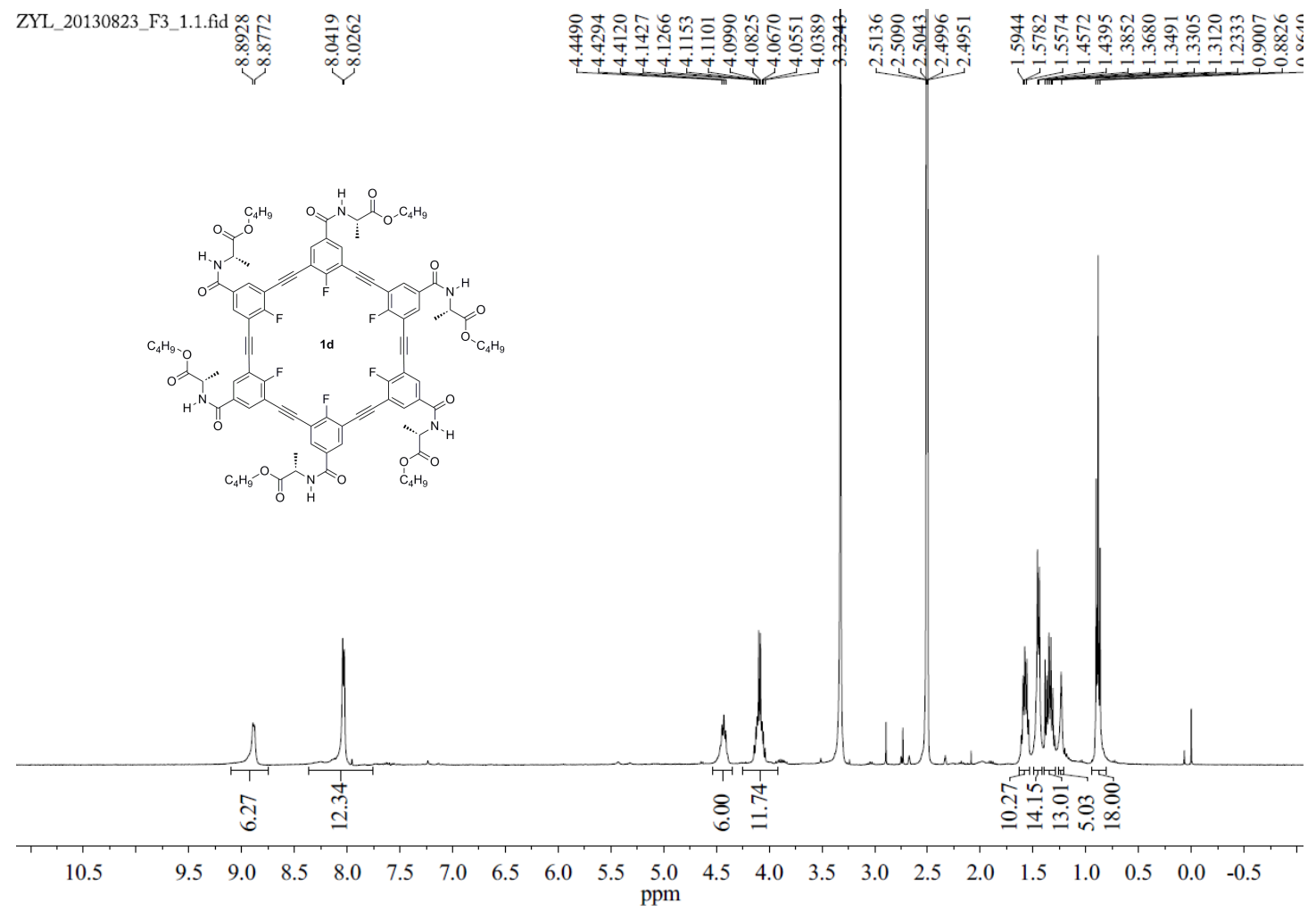

Figure S7 ${ }^{1} \mathrm{H}$ NMR Spectra of macrocycle1d (2 mM, DMSO- $\left.d_{6}\right)$

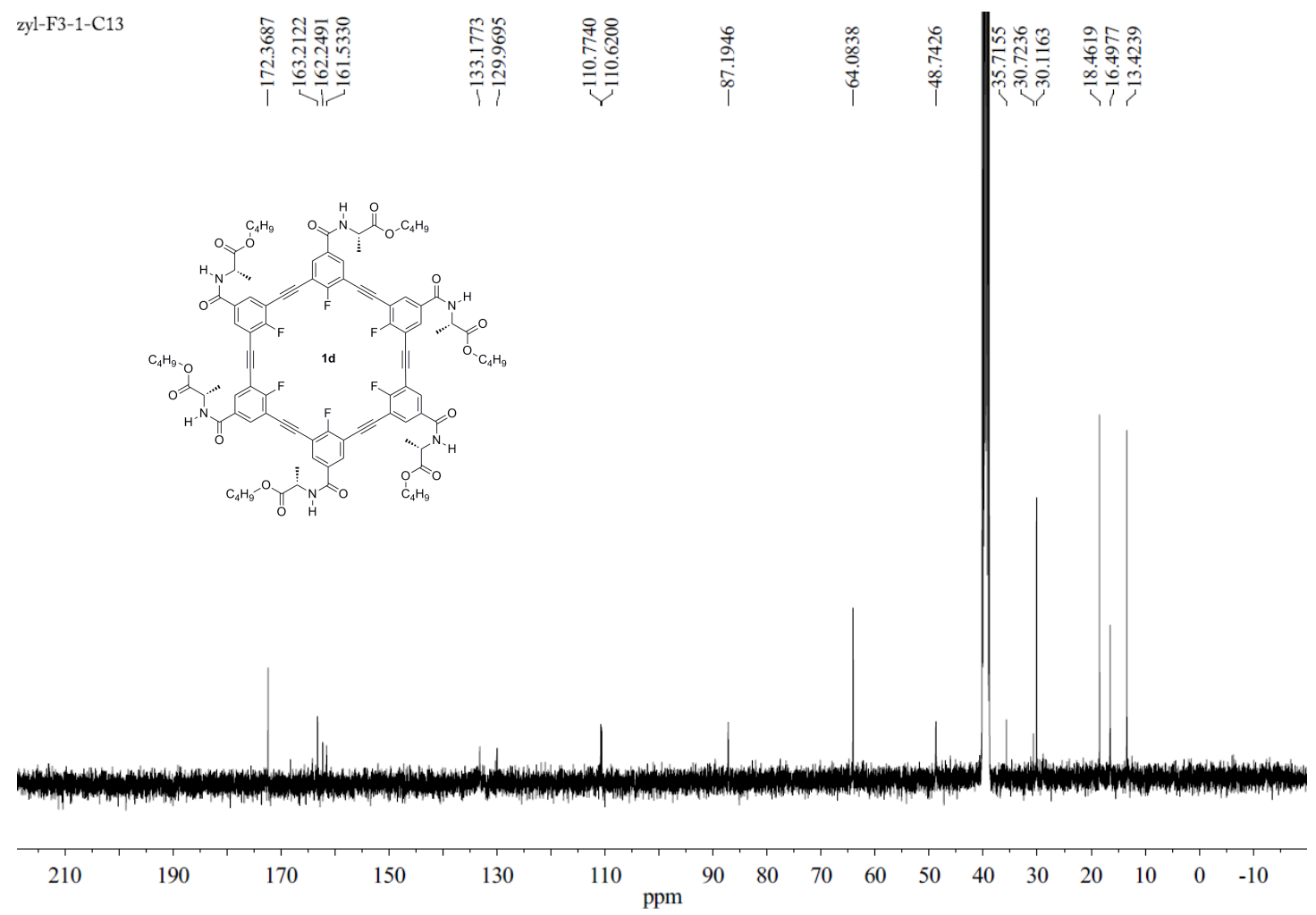

Figure S8 ${ }^{13} \mathrm{C}$ NMR Spectra of macrocycle 1d (4 mM, DMSO- $\left.d_{6}\right)$ 

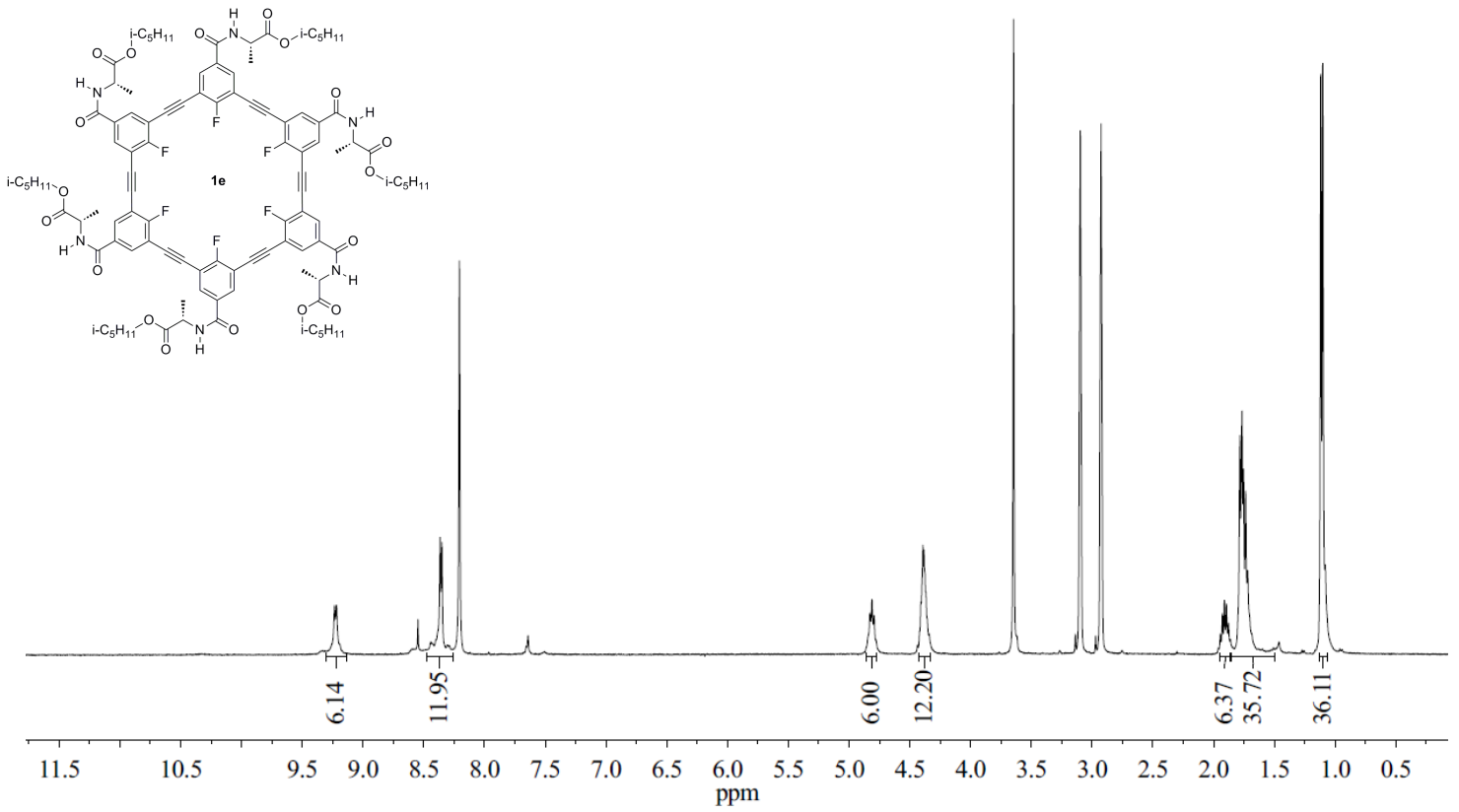

Figure S9 ${ }^{1} \mathrm{H}$ NMR Spectra of macrocycle 1e (2 mM, DMF- $\left.d_{7}\right)$

wqh_F3a-13C

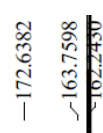

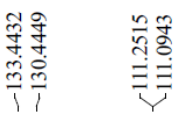

$\infty$
0
$\infty$
$\infty$
$\infty$
1

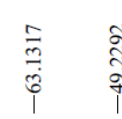
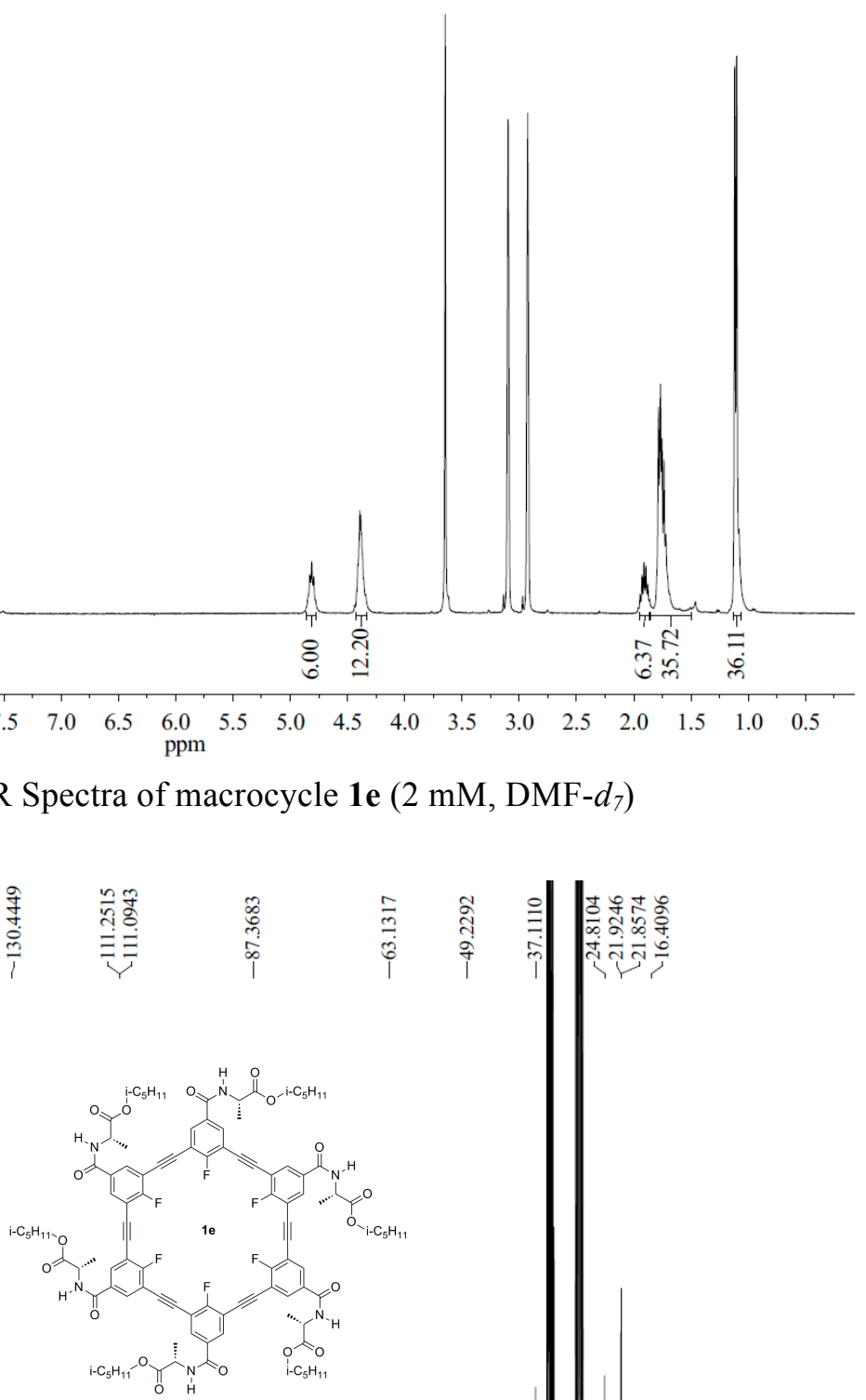


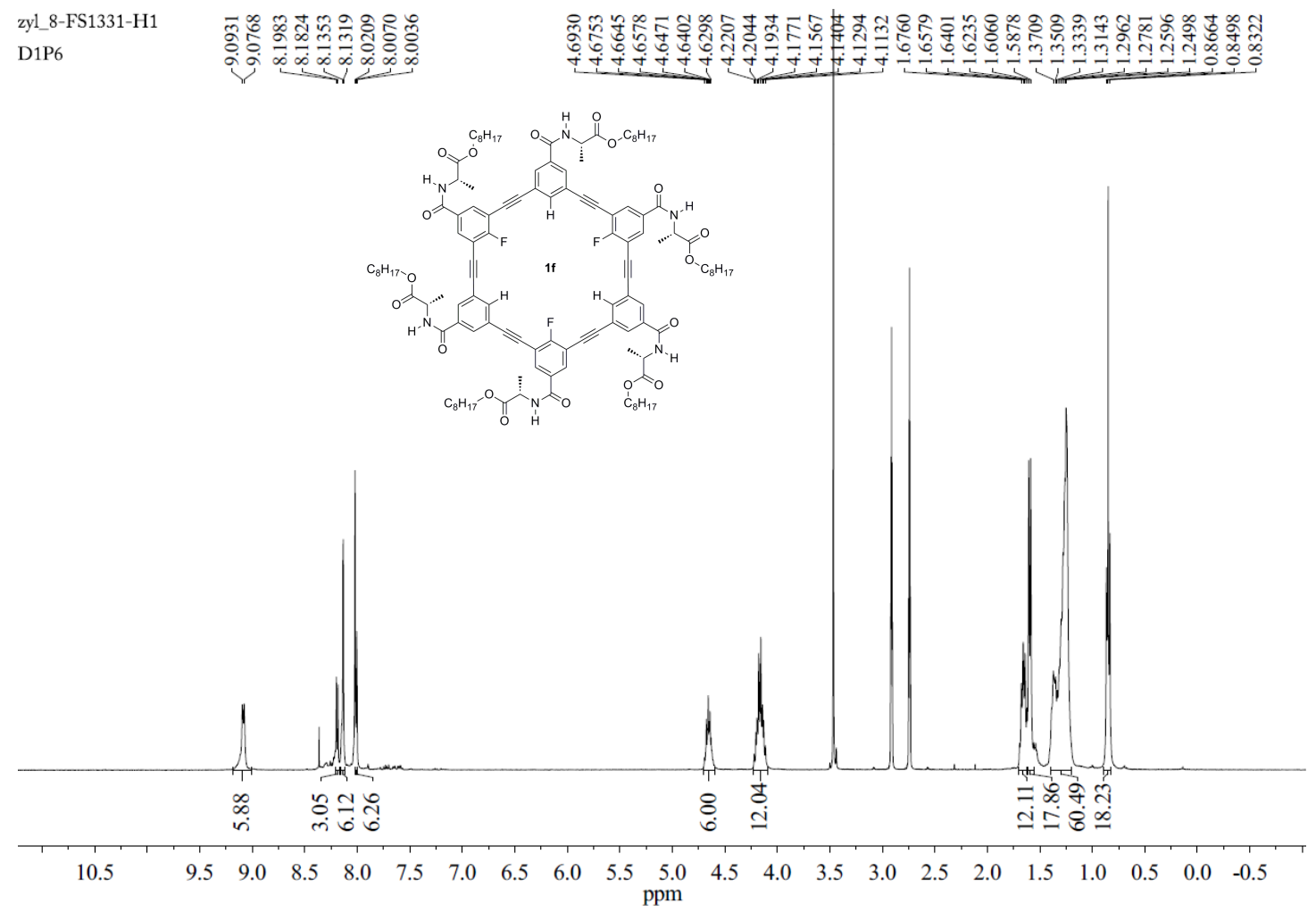

Figure S11 ${ }^{1} \mathrm{H}$ NMR Spectra of macrocycle 1 (f (2 mM, DMF- $\left.d_{7}\right)$

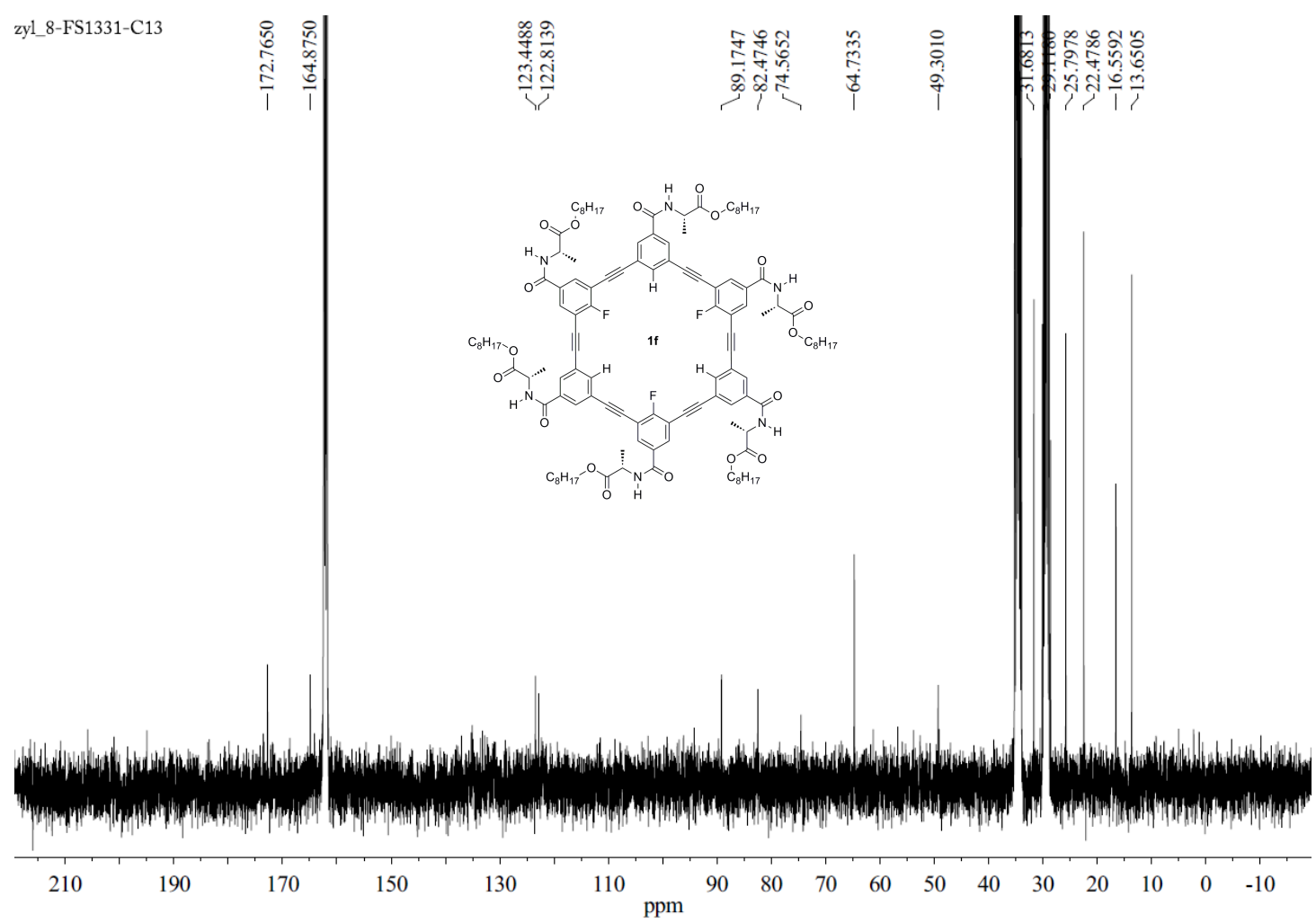

Figure S12 ${ }^{13} \mathrm{C}$ NMR Spectra of macrocycle 1 (f $\left(4 \mathrm{mM}\right.$, DMF- $\left.d_{7}\right)$ 


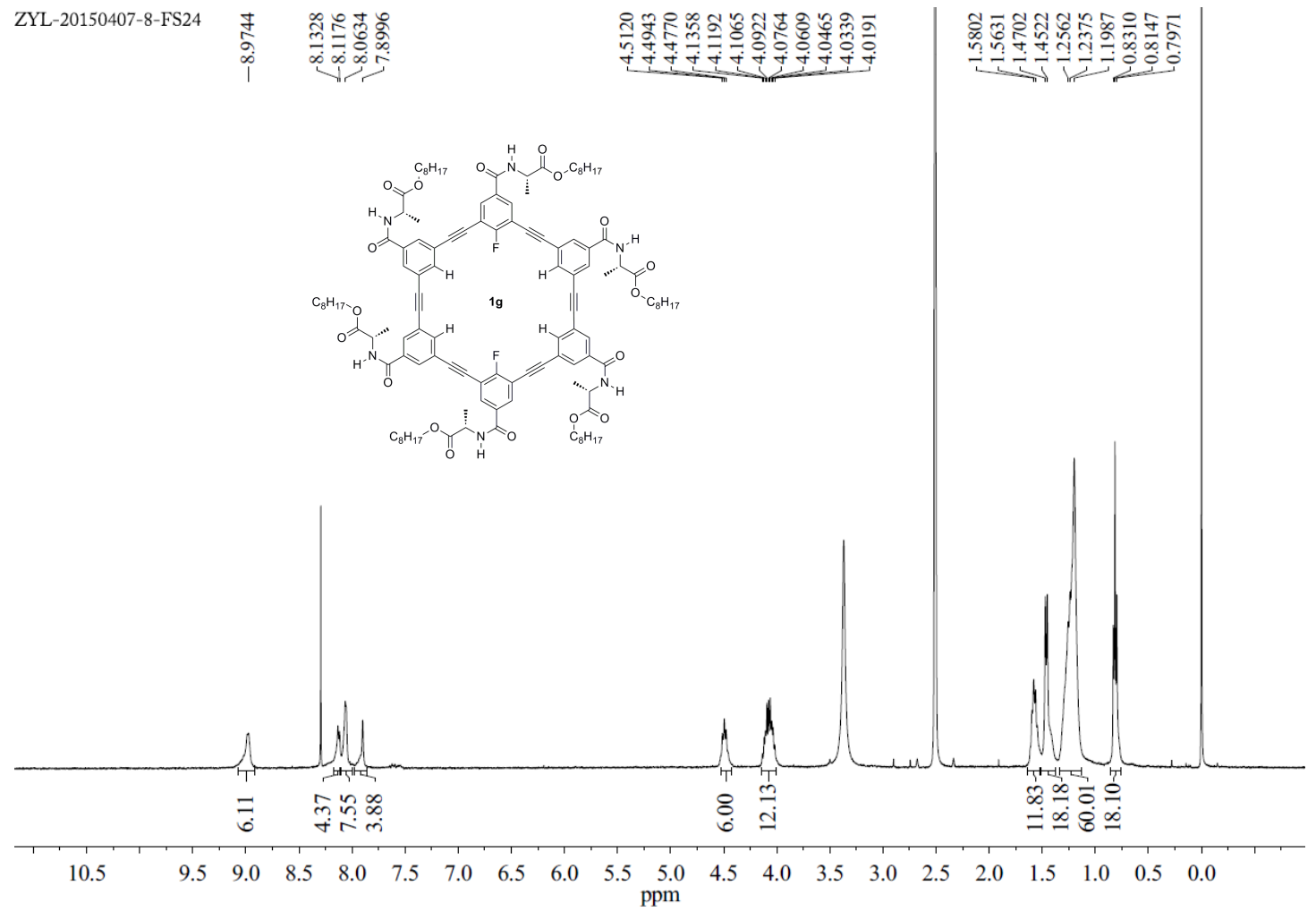

Figure S13 ${ }^{1} \mathrm{H}$ NMR Spectra of macrocycle $1 \mathrm{~g}\left(2 \mathrm{mM}, 10 \% \mathrm{CDCl}_{3}+\mathrm{DMSO}_{-} d_{6}\right)$

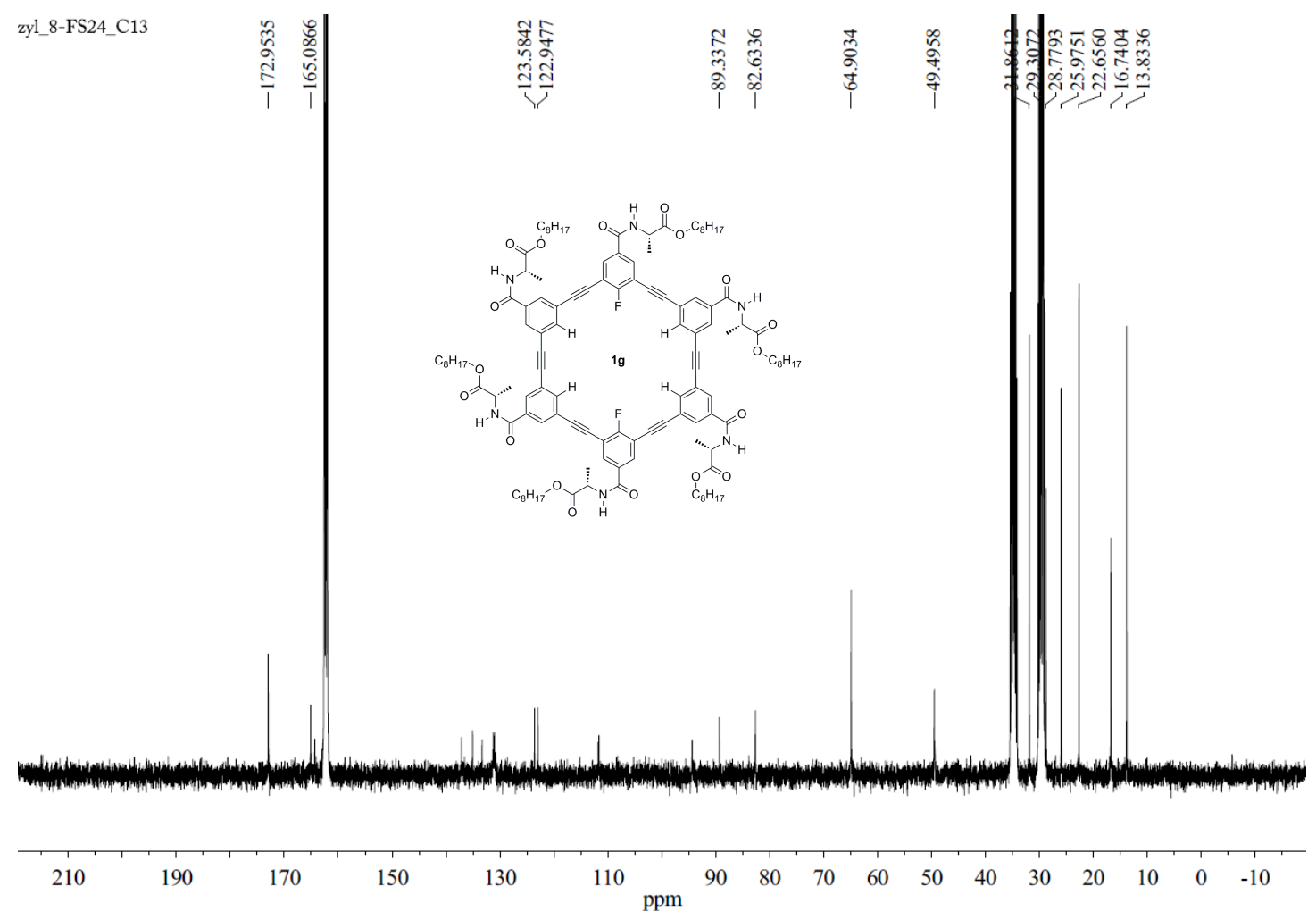

Figure S14 ${ }^{13} \mathrm{C}$ NMR Spectra of macrocycle $1 \mathrm{~g}$ (4 mM, DMF- $\left.d_{7}\right)$ 


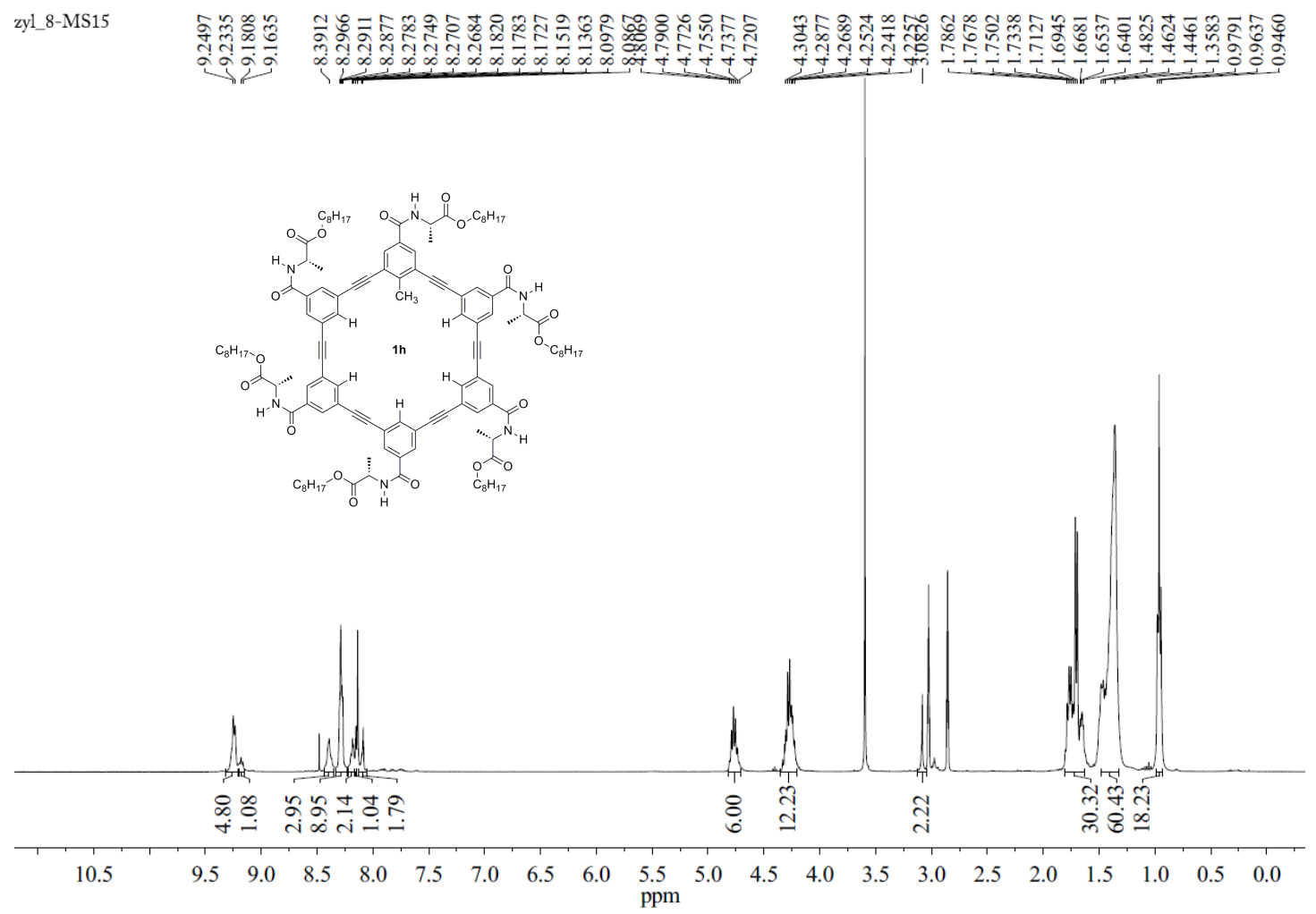

Figure S15 ${ }^{1} \mathrm{H}$ NMR Spectra of macrocycle1h $\left(2 \mathrm{mM}\right.$, DMF- $\left.d_{7}\right)$
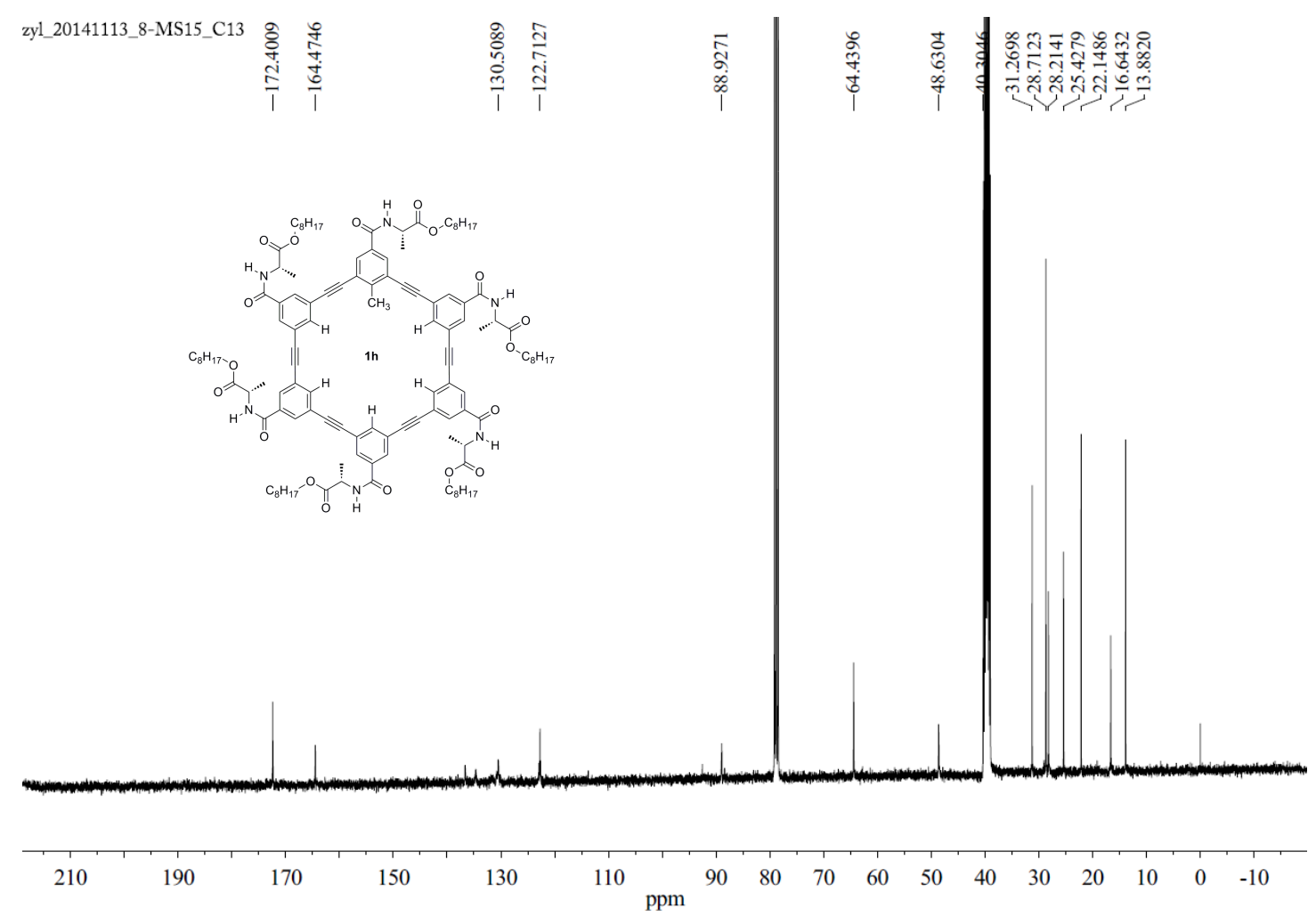

Figure S16 ${ }^{13} \mathrm{C}$ NMR Spectra of macrocycle $1 \mathrm{~h}\left(4 \mathrm{mM}, 20 \% \mathrm{CDCl}_{3}+\mathrm{DMSO}-d_{6}\right)$ 
zyl_8-MS24
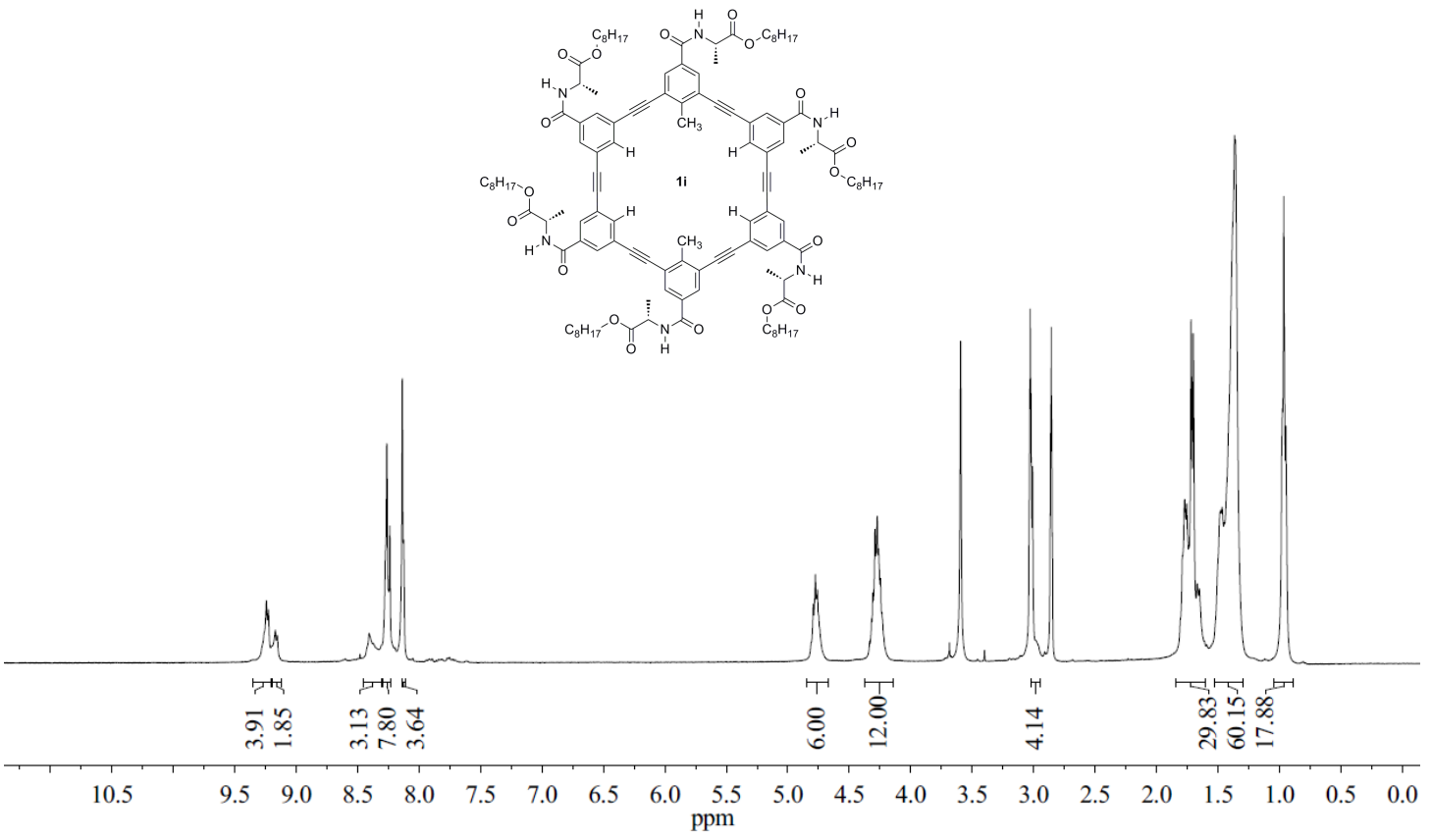

Figure S17 ${ }^{1} \mathrm{H}$ NMR Spectra of macrocycle1i (4 mM, DMF- $\left.d_{7}\right)$
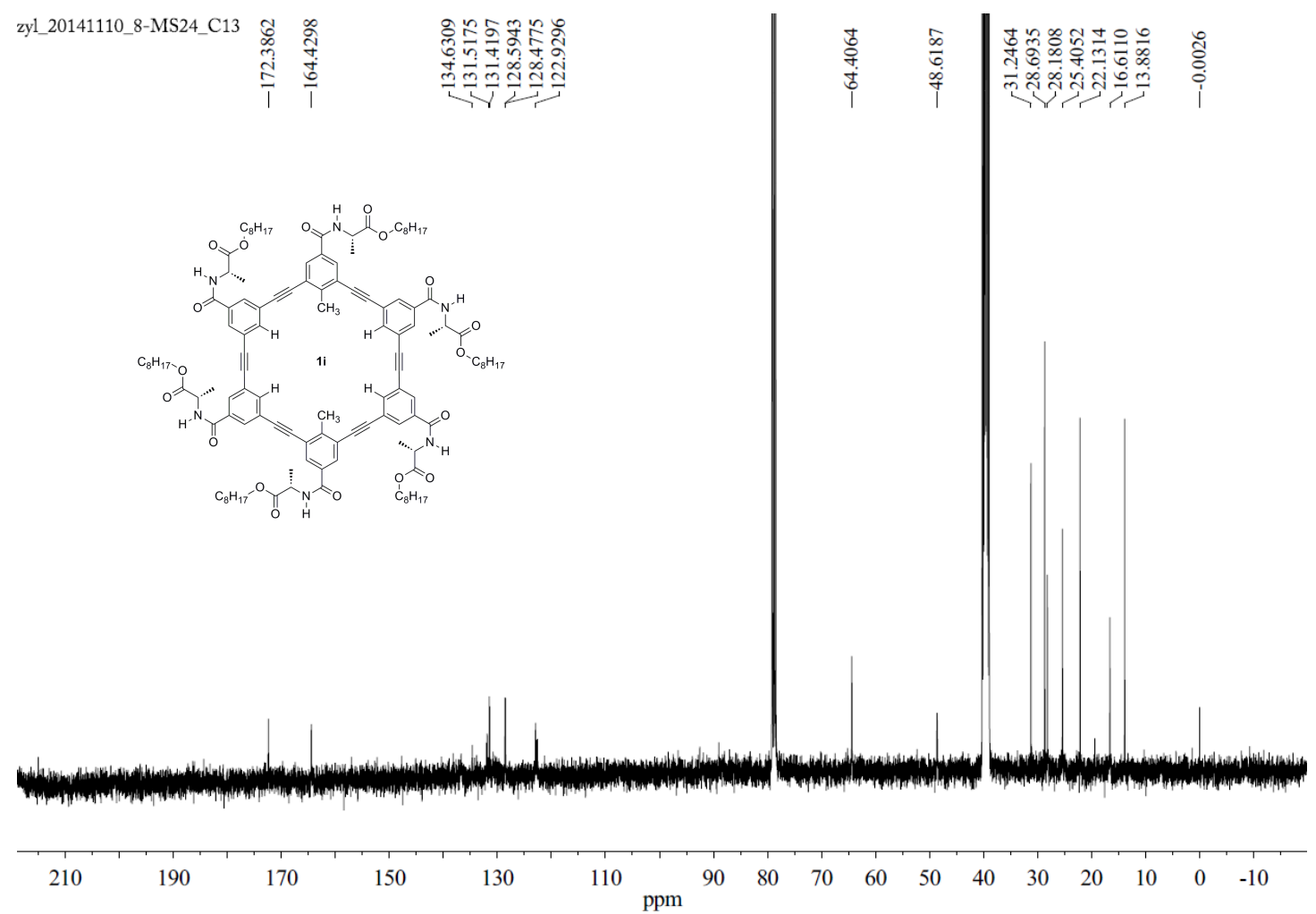

Figure S18 ${ }^{13} \mathrm{C}$ NMR Spectra of macrocycle1i (4 mM, 20\% $\left.\mathrm{CDCl}_{3}+\mathrm{DMSO}_{-} d_{6}\right)$ 


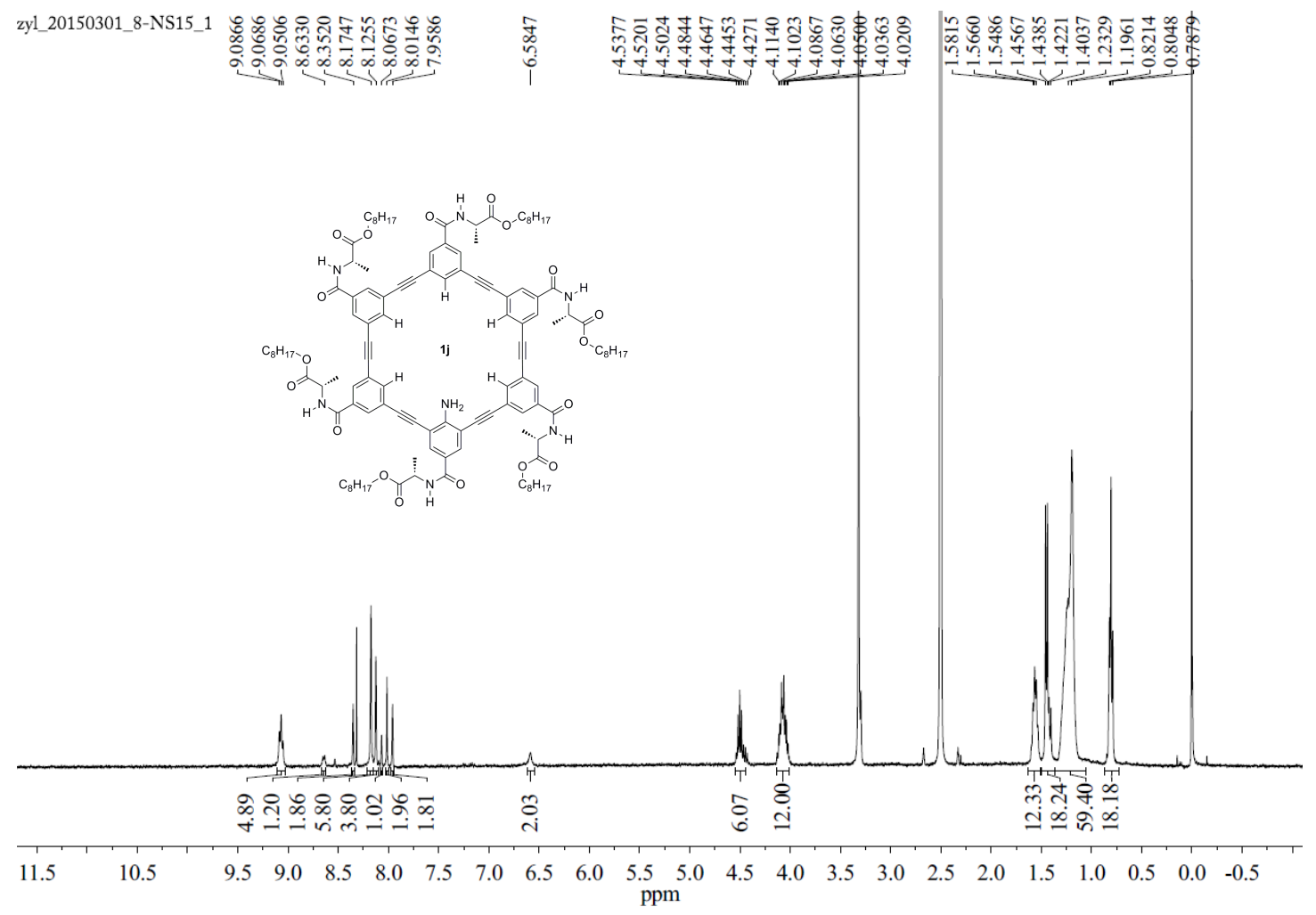

Figure S19 ${ }^{1} \mathrm{H}$ NMR Spectra of macrocycle $\mathbf{1 j}\left(2 \mathrm{mM}, 10 \% \mathrm{CDCl}_{3}+\mathrm{DMSO}_{6}\right)$

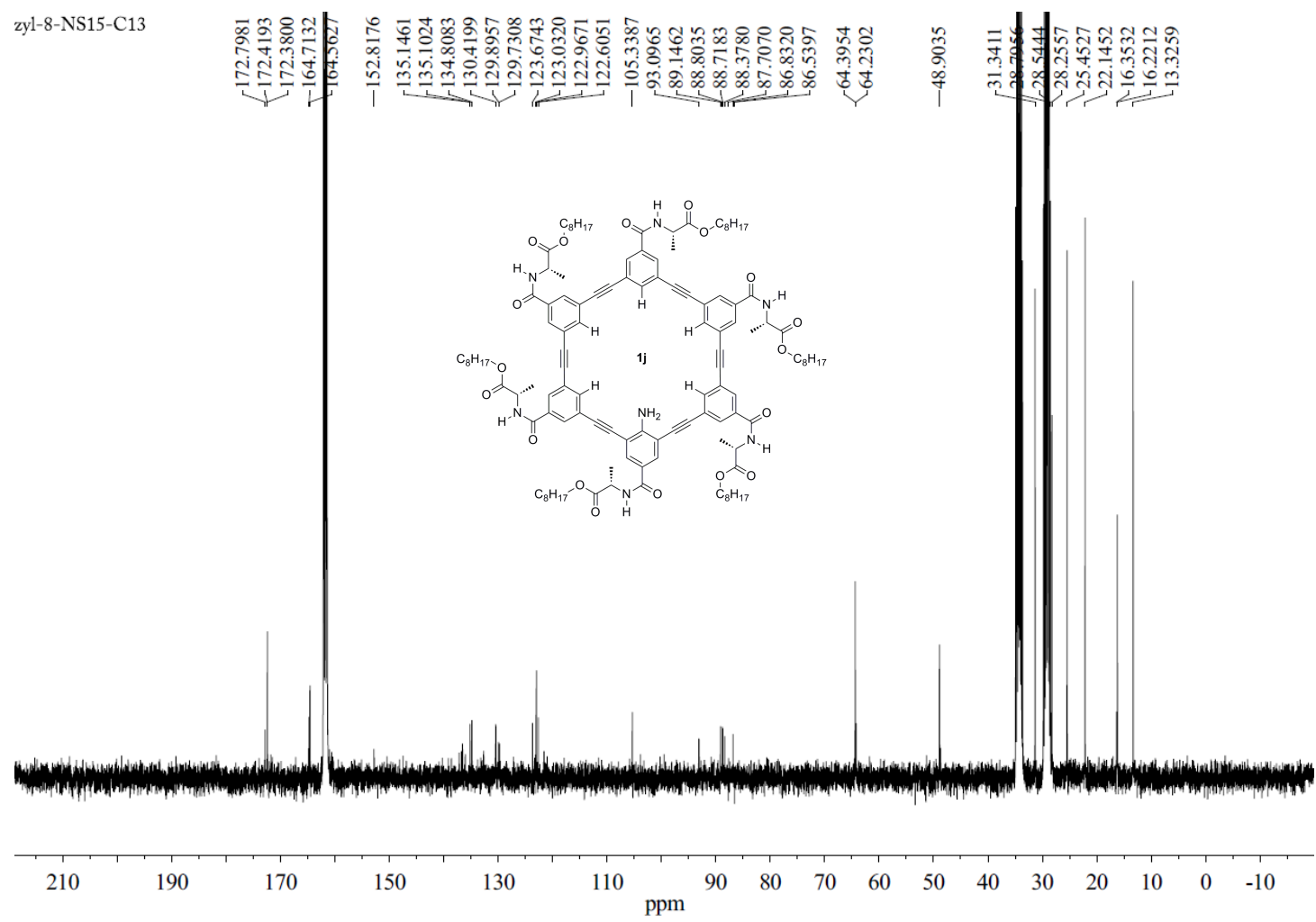

Figure S20 ${ }^{13} \mathrm{C}$ NMR Spectra of macrocycle1j (4 mM, DMF- $\left.d_{7}\right)$ 
zyl_8-NS24
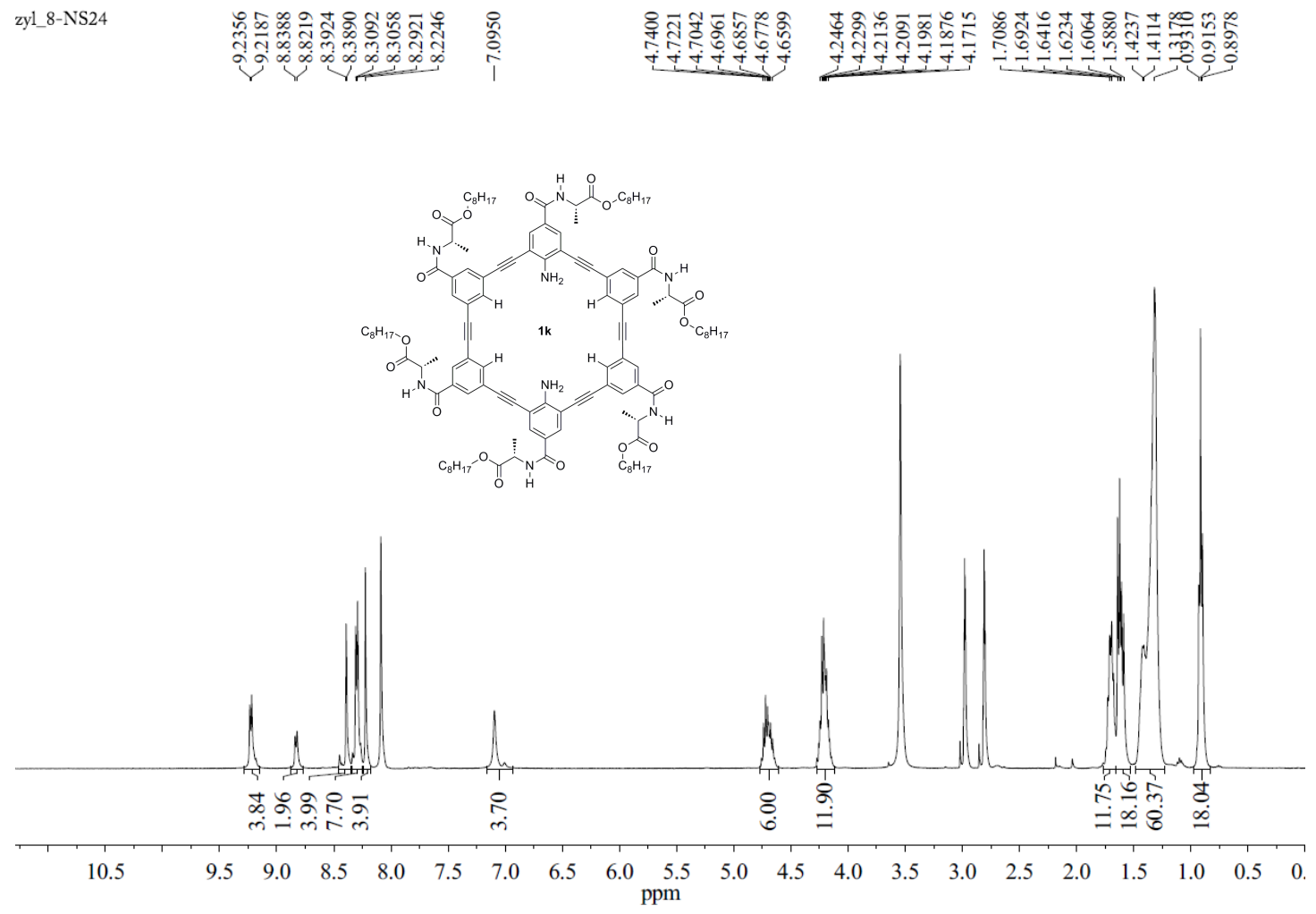

Figure S21 ${ }^{1}$ H NMR Spectra of macrocycle1k (2 mM, DMF- $\left.d_{7}\right)$

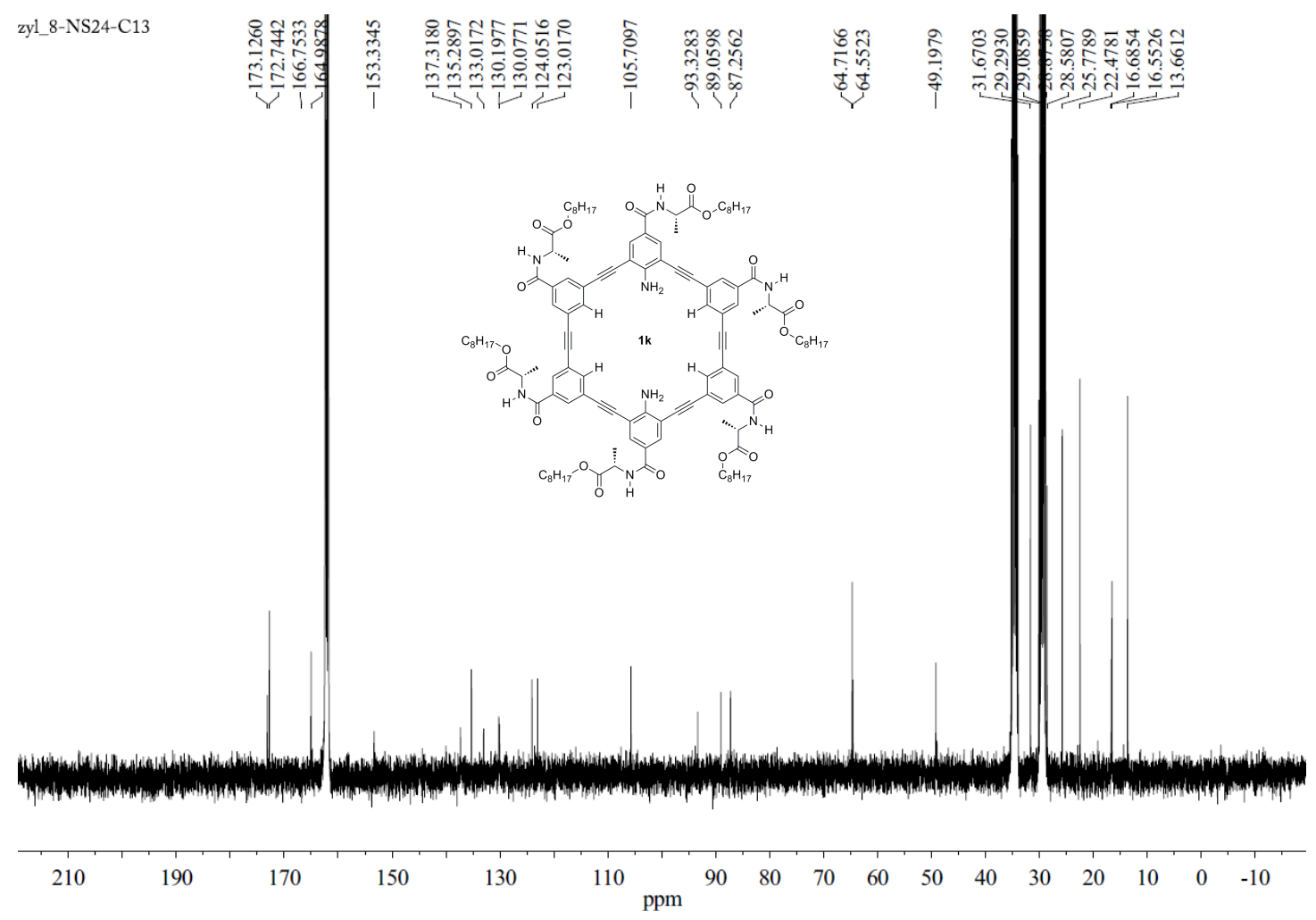

Figure S22 ${ }^{13} \mathrm{C}$ NMR Spectra of macrocycle1k (4 mM, DMF- $d_{7}$ ) 
zyl_8-FN14
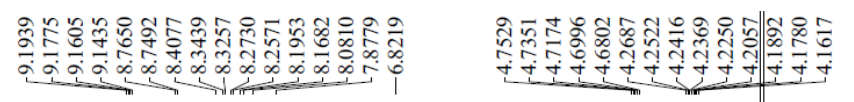

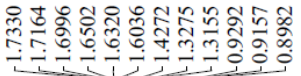
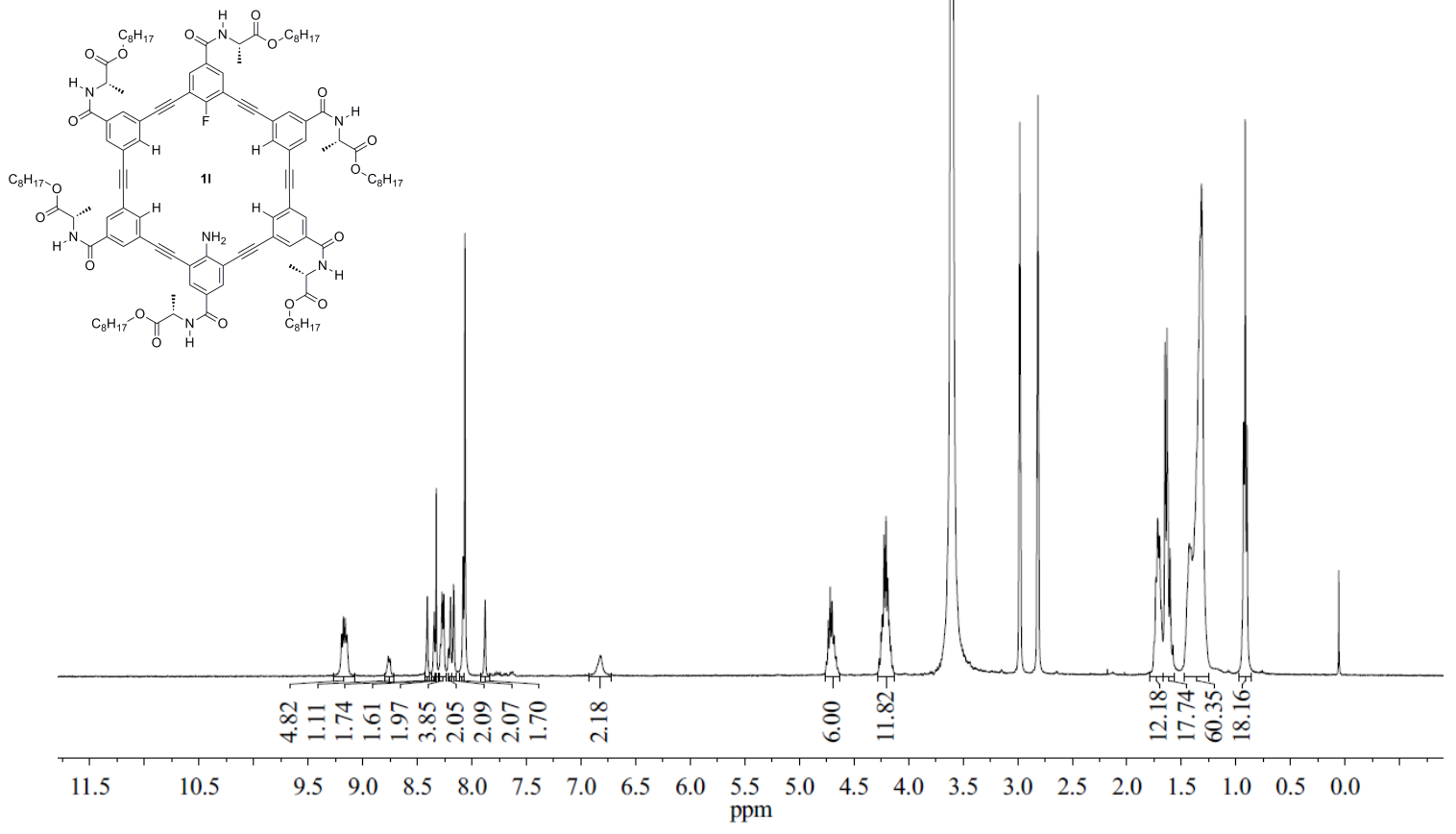

Figure S23 ${ }^{13} \mathrm{C}$ NMR Spectra of macrocycle $1 \mathrm{~g}$ (4 mM, DMF- $\left.d_{7}\right)$

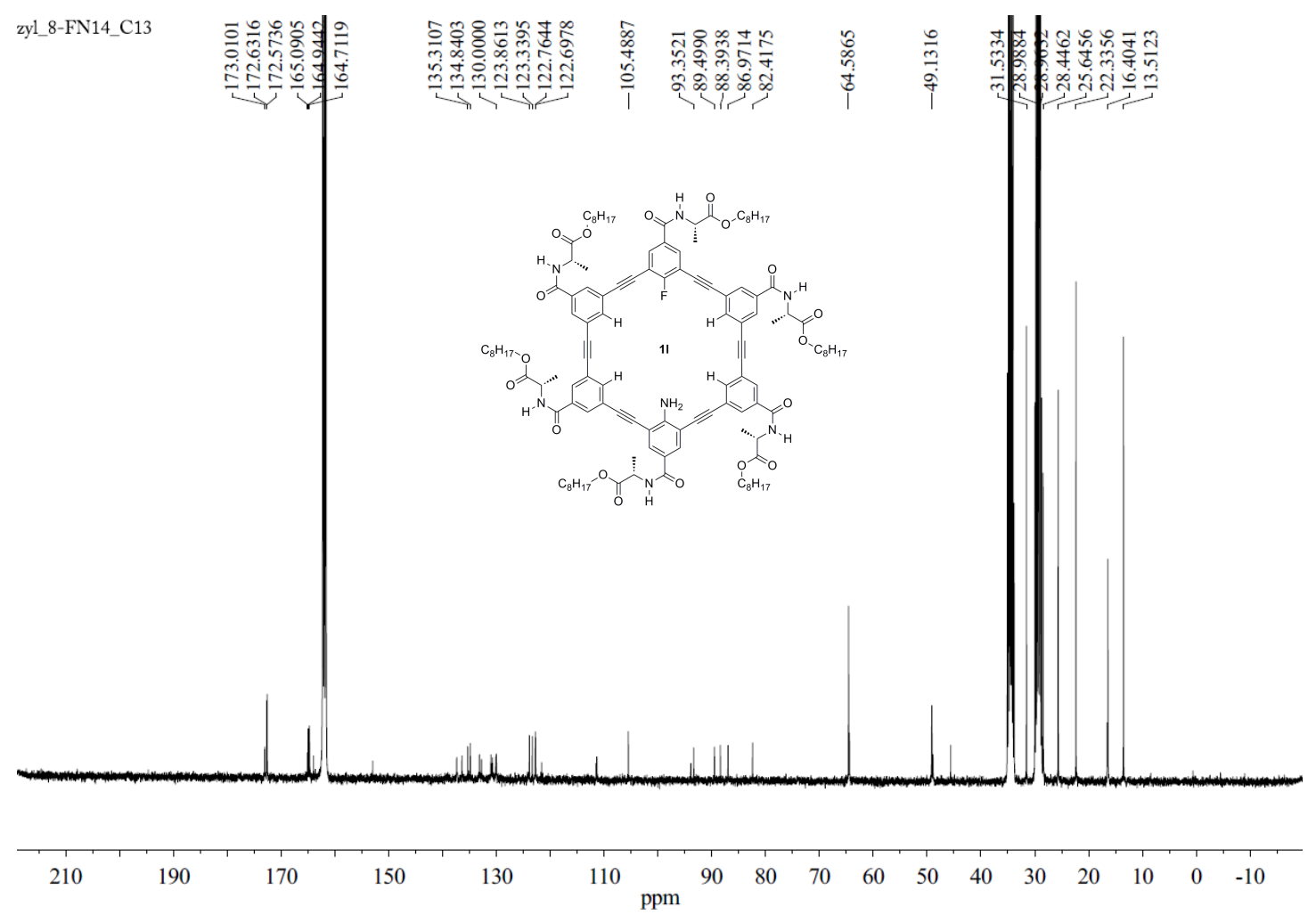

Figure S24 ${ }^{13} \mathrm{C}$ NMR Spectra of macrocycle 11 (4 mM, DMF- $\left.d_{7}\right)$ 


\section{Mass Spectra of macrocycles 1a-I}

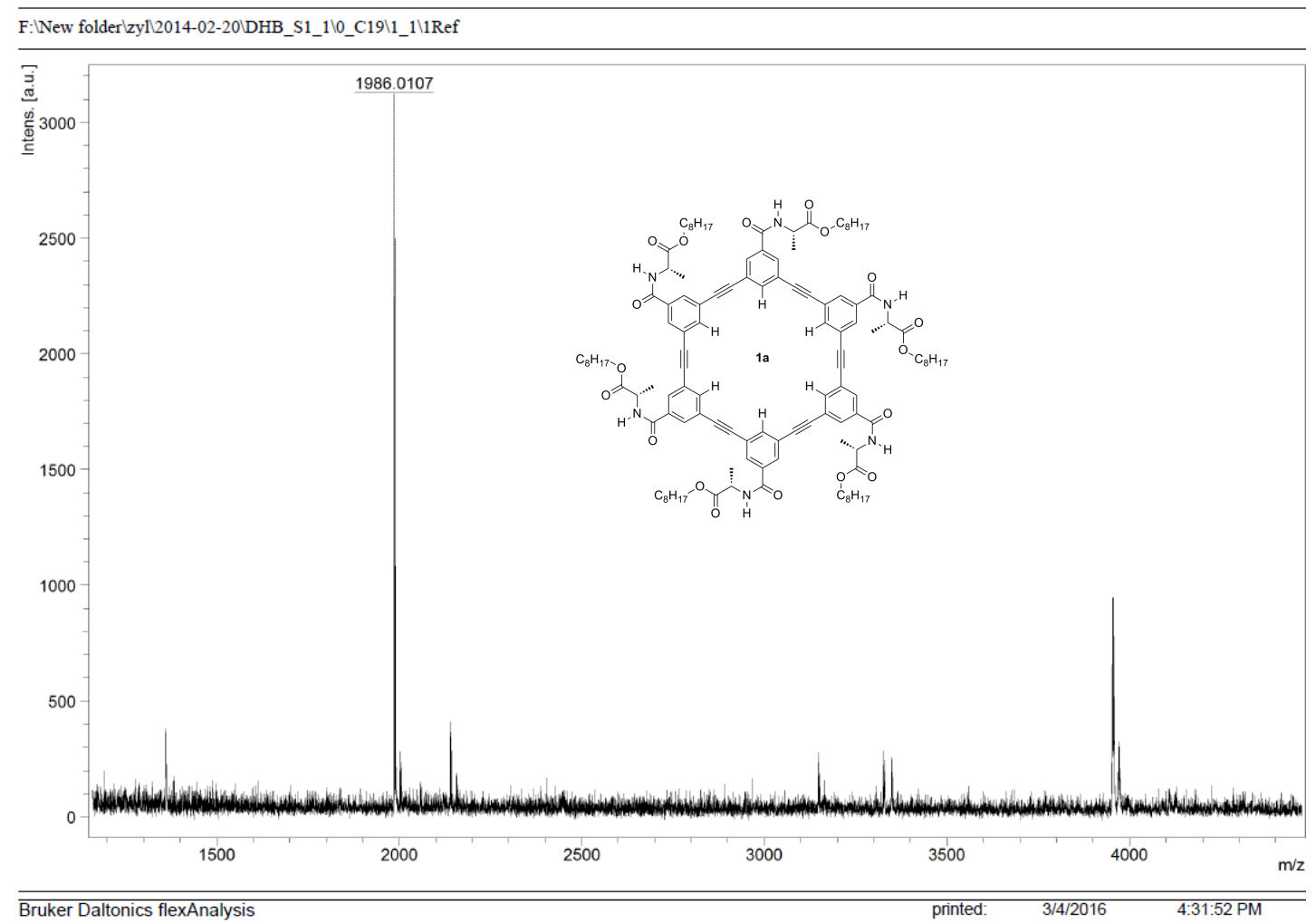

Figure S25 Mass Spectra of macrocycle1a

\begin{tabular}{|c|c|c|c|c|c|c|}
\hline Formula & Mass & Error & mSigma & $\mathrm{Db} 1 \mathrm{Eq}$ & $\mathrm{N}$ rule & $\begin{array}{c}\text { Electron } \\
\text { Configuration }\end{array}$ \\
\hline $\begin{array}{llllllll}\text { C } 120 & \text { H } & 150 & \text { N } & 6 & \text { Na } & 0 & 18\end{array}$ & $1,986.0899$ & 39. 8847 & 685.3103 & 48.50 & ok & even \\
\hline
\end{tabular}




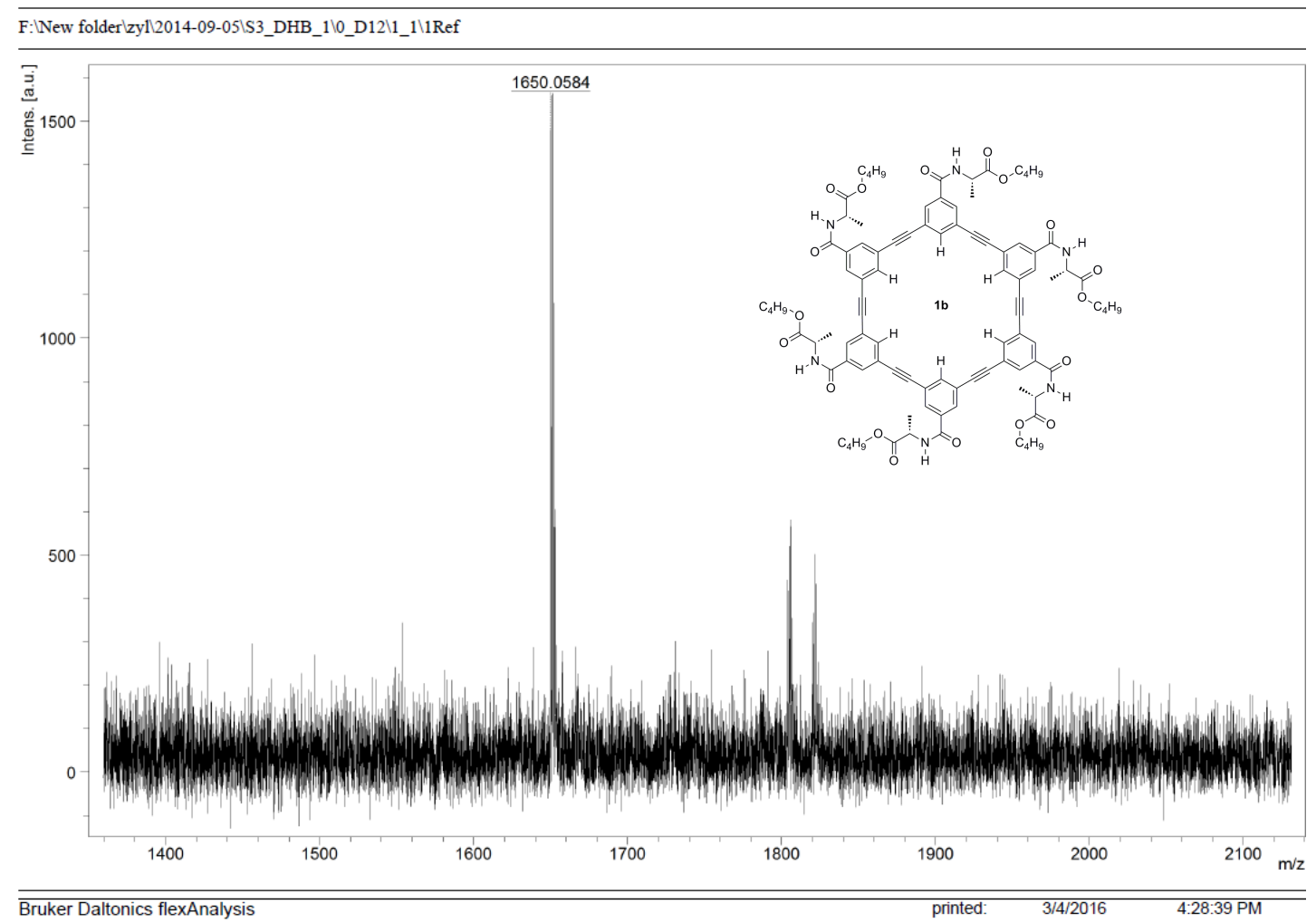

Figure S26Mass Spectra of macrocycle1b

\begin{tabular}{cccccccc}
\hline \multicolumn{2}{c}{ Formula } & Mass & Error & mSigma & DblEq & N rule & $\begin{array}{c}\text { Electron } \\
\text { Configuration }\end{array}$ \\
\hline C $96 \quad$ H $102 \mathrm{~N} 6$ Na 018 & $1,649.7143$ & 208.5543 & 718.5332 & 48.50 & ok & even \\
\hline
\end{tabular}




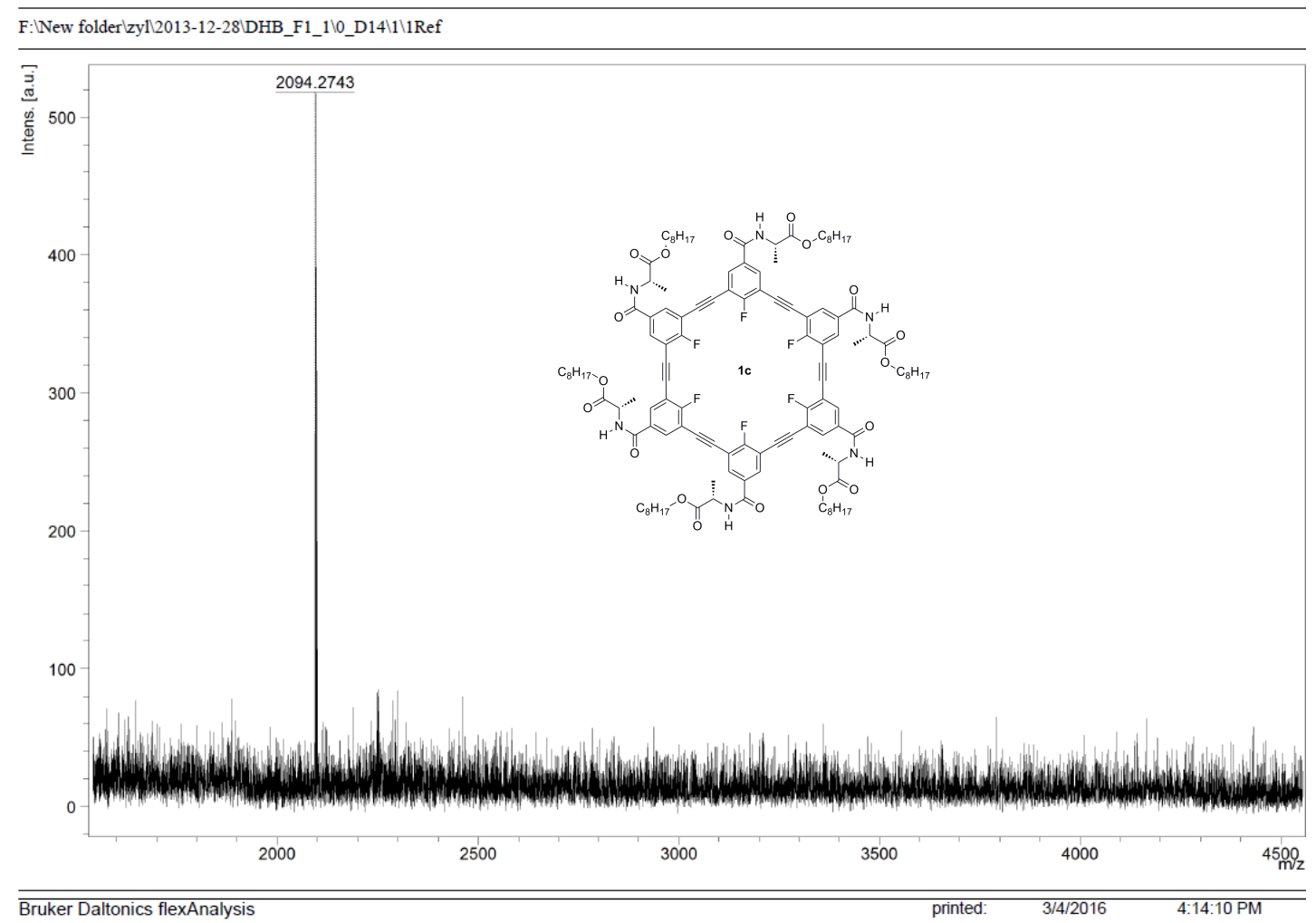

Figure S27Mass Spectra of macrocycle1c

\begin{tabular}{lcccccc}
\hline \multicolumn{1}{c}{ Formula } & Mass & Error & mSigma & Db1Eq & N rule & $\begin{array}{c}\text { Electron } \\
\text { Configuration }\end{array}$ \\
\hline $\begin{array}{l}\text { C 120 H 144 F 6 N 6 Na O } \\
18\end{array}$ & $2,094.0334$ & 115.0720 & 685.4020 & 48.50 & ok & even \\
\hline
\end{tabular}




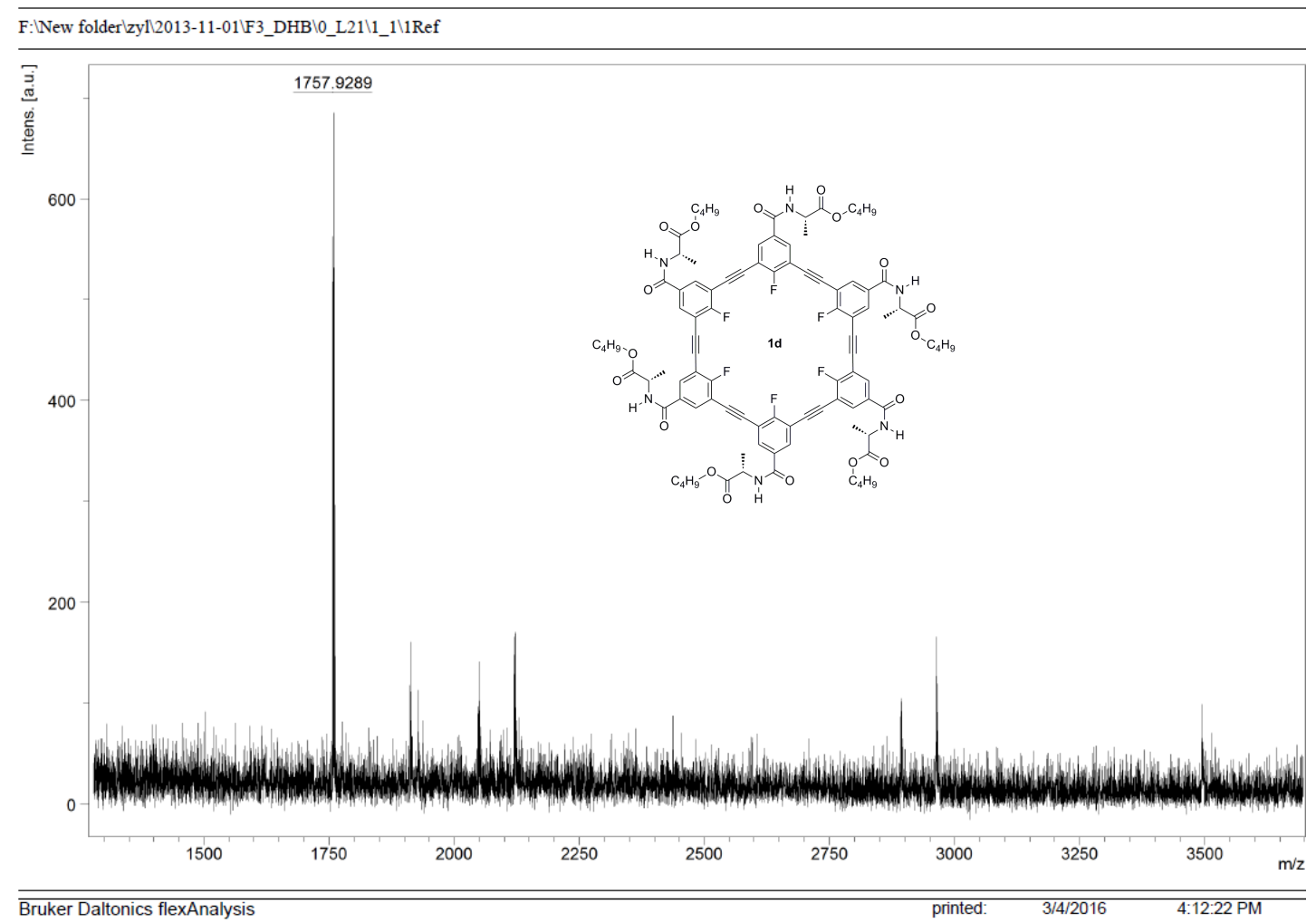

Figure S28Mass Spectra of macrocycle1d

\begin{tabular}{ccccccc}
\hline Formula & Mass & Error & mSigma & DblEq & N rule & $\begin{array}{c}\text { Electron } \\
\text { Configuration }\end{array}$ \\
\hline C 96 H 96 F 6 N 6 Na O 18 & $1,757.6578$ & 154.2438 & 718.6205 & 48.50 & ok & even \\
\hline
\end{tabular}




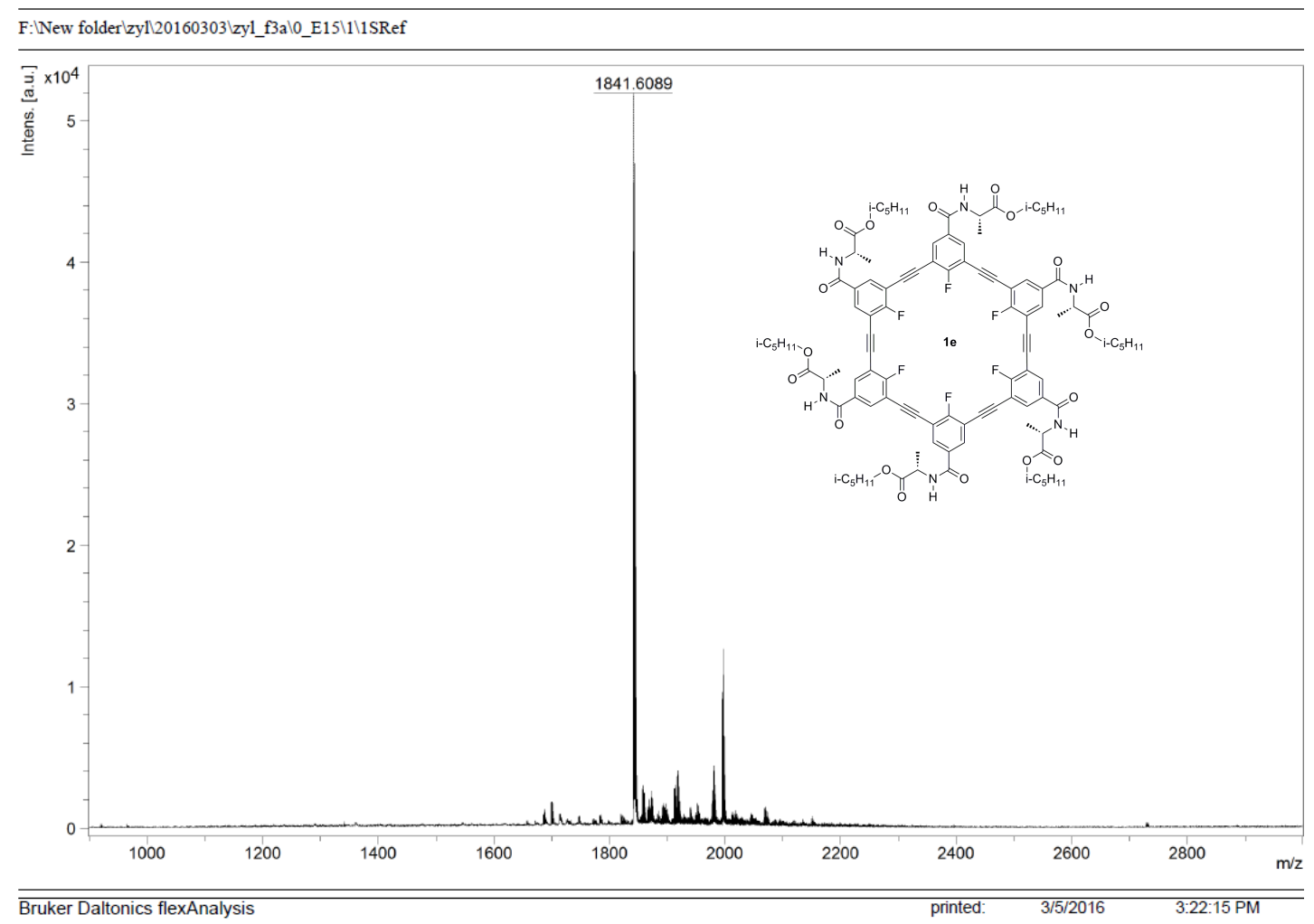

Figure S29Mass Spectra of macrocycle1e

\begin{tabular}{lcccccc}
\hline Formula & Mass & Error & mSigma & Db1Eq & N rule & $\begin{array}{c}\text { Electron } \\
\text { Configuration }\end{array}$ \\
\hline $\begin{array}{l}\text { C 102 H 108 F 6 N 6 Na O } \\
18\end{array}$ & $1,841.7517$ & 77.4887 & 710.4324 & 48.50 & ok & even \\
\hline
\end{tabular}




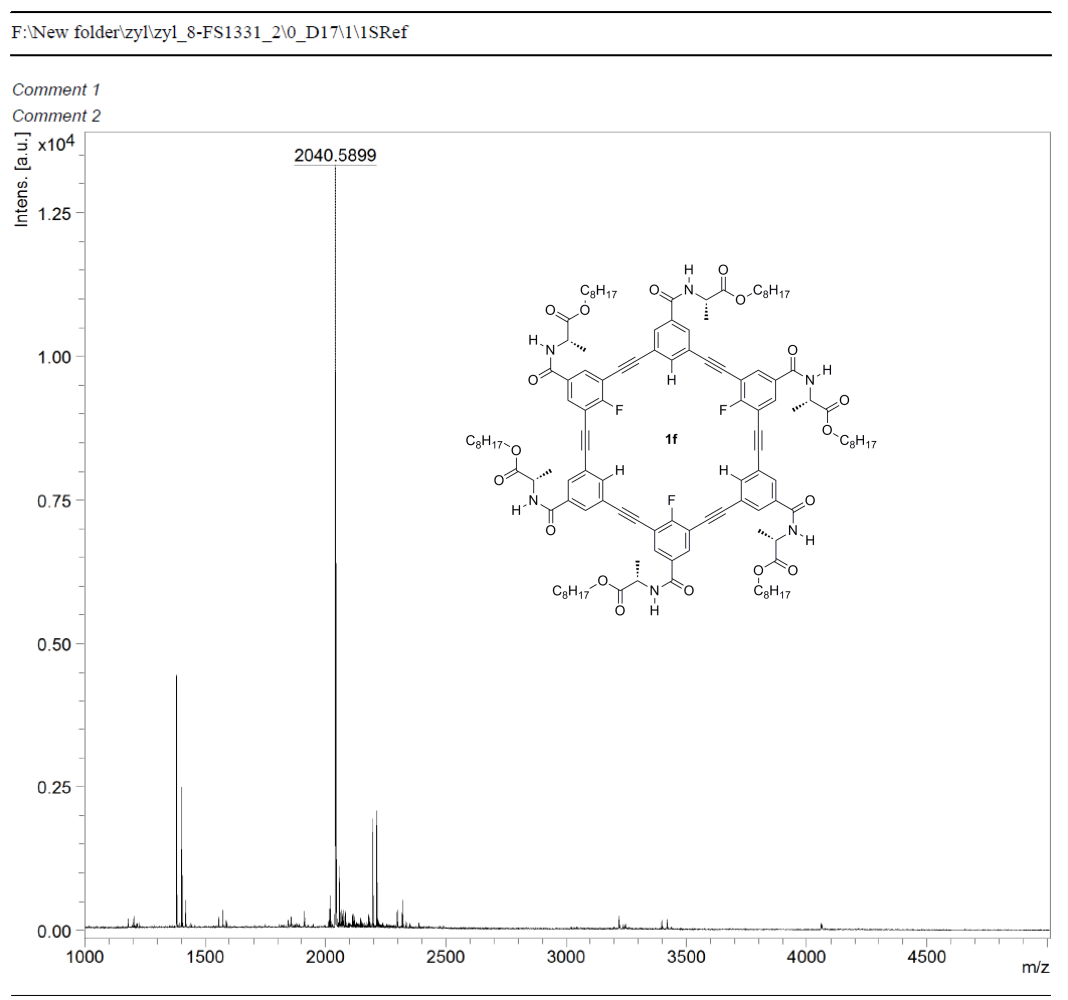

Figure S30Mass Spectra of macrocycle1f

\begin{tabular}{lcccccc}
\hline \multicolumn{1}{c}{ Formula } & Mass & Error & mSigma & DblEq & N rule & $\begin{array}{c}\text { Electron } \\
\text { Configuration }\end{array}$ \\
\hline $\begin{array}{l}\text { C 120 H 147 F 3 N 6 Na O } \\
18\end{array}$ & $2,040.0616$ & 258.8728 & 685.3561 & 48.50 & ok & even \\
\hline
\end{tabular}




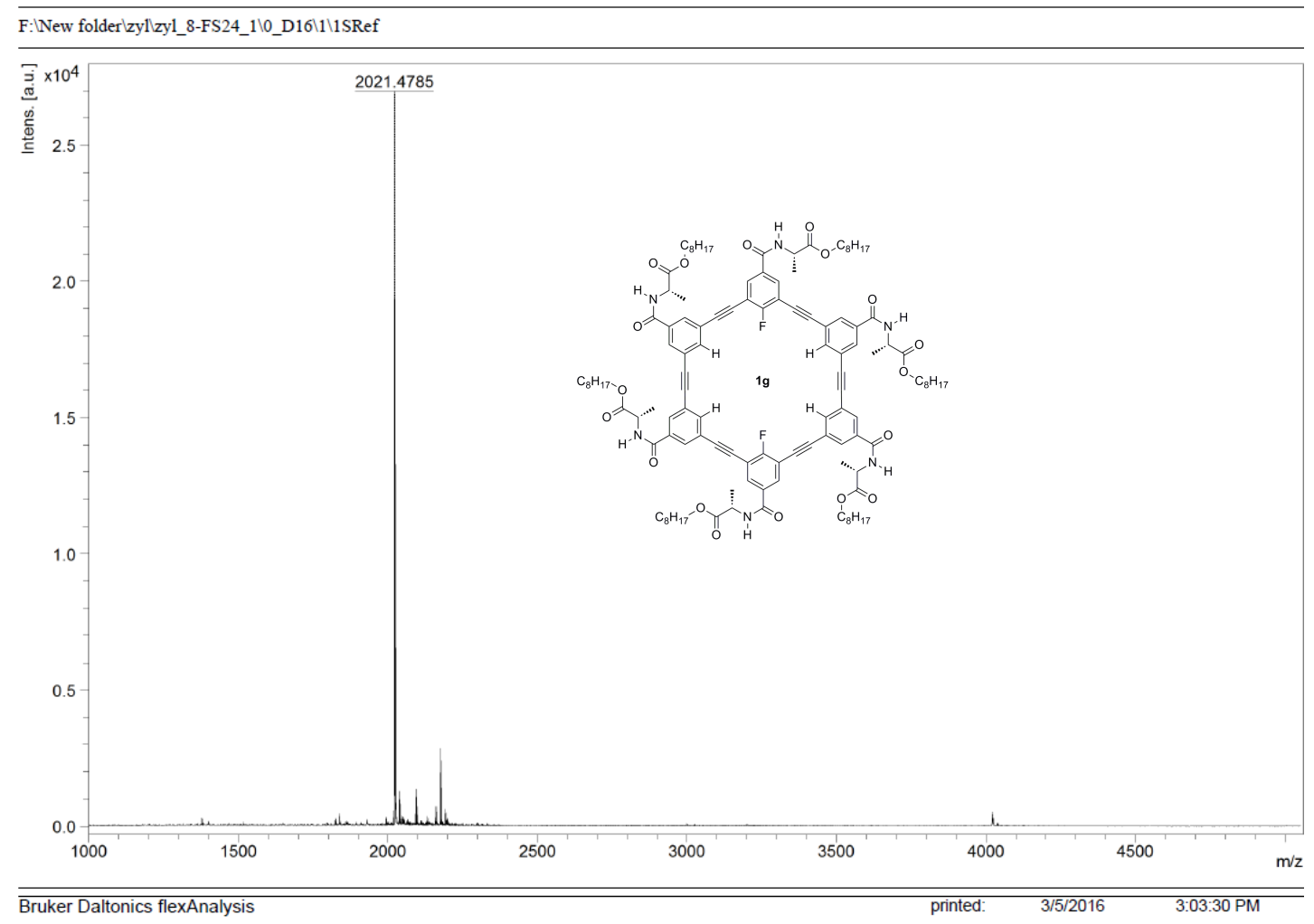

Figure S31Mass Spectra of macrocycle1g

\begin{tabular}{lcccccc}
\hline Formula & Mass & Error & mSigma & DblEq & N rule & $\begin{array}{c}\text { Electron } \\
\text { Configuration }\end{array}$ \\
\hline $\begin{array}{l}\text { C 120 H 148 F 2 N 6 Na O } \\
18\end{array}$ & $2,022.0710$ & 293.1365 & NaN & 48.50 & ok & even \\
\hline
\end{tabular}




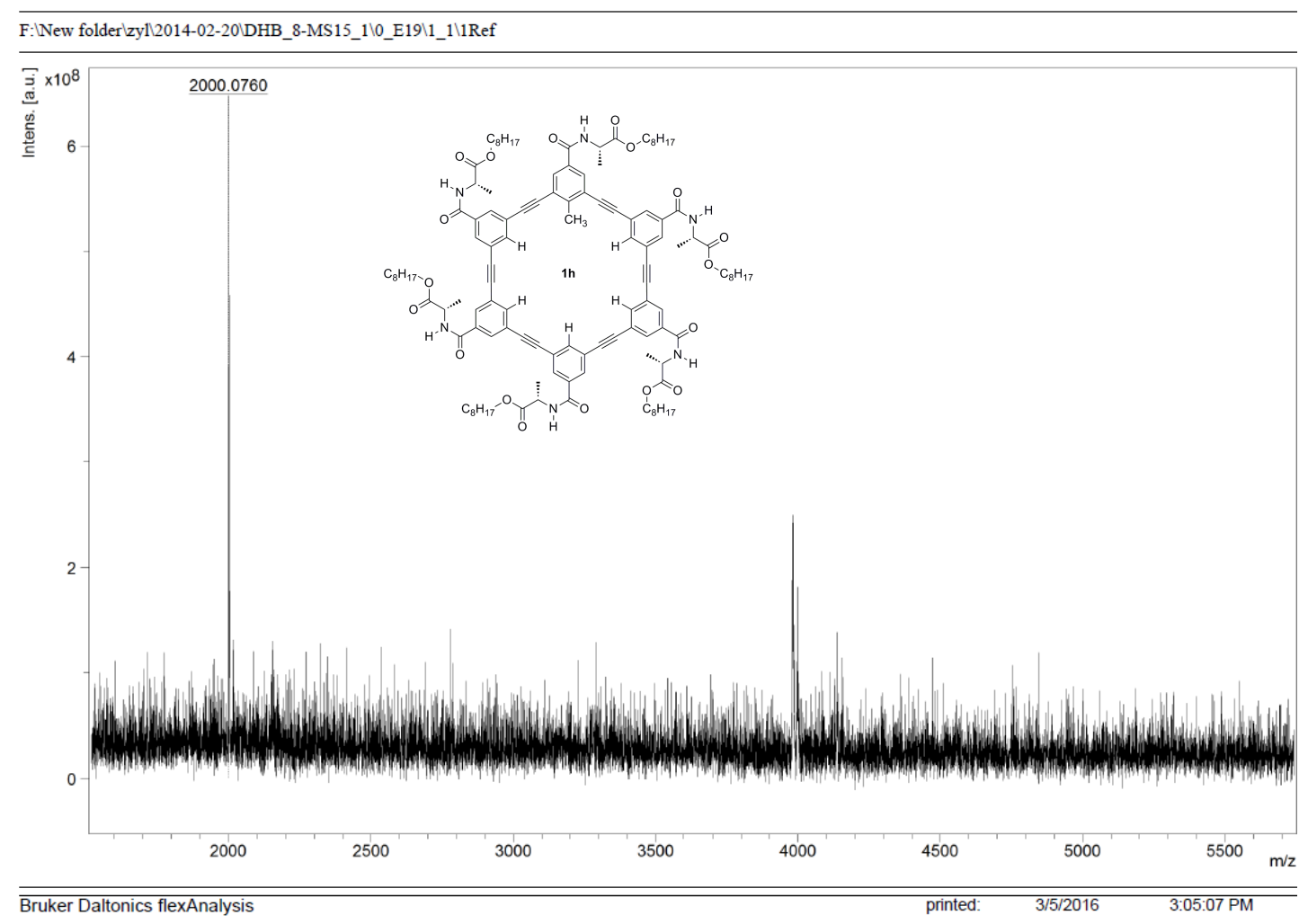

Figure S32Mass Spectra of macrocycle1h

\begin{tabular}{ccccccc}
\hline Formula & Mass & Error & mSigma & DblEq & N rule & $\begin{array}{c}\text { Electron } \\
\text { Configuration }\end{array}$ \\
\hline C 121 H 152 N 6 Na O 18 & $2,000.1055$ & 14.7576 & 683.8624 & 48.50 & ok & even \\
\hline
\end{tabular}




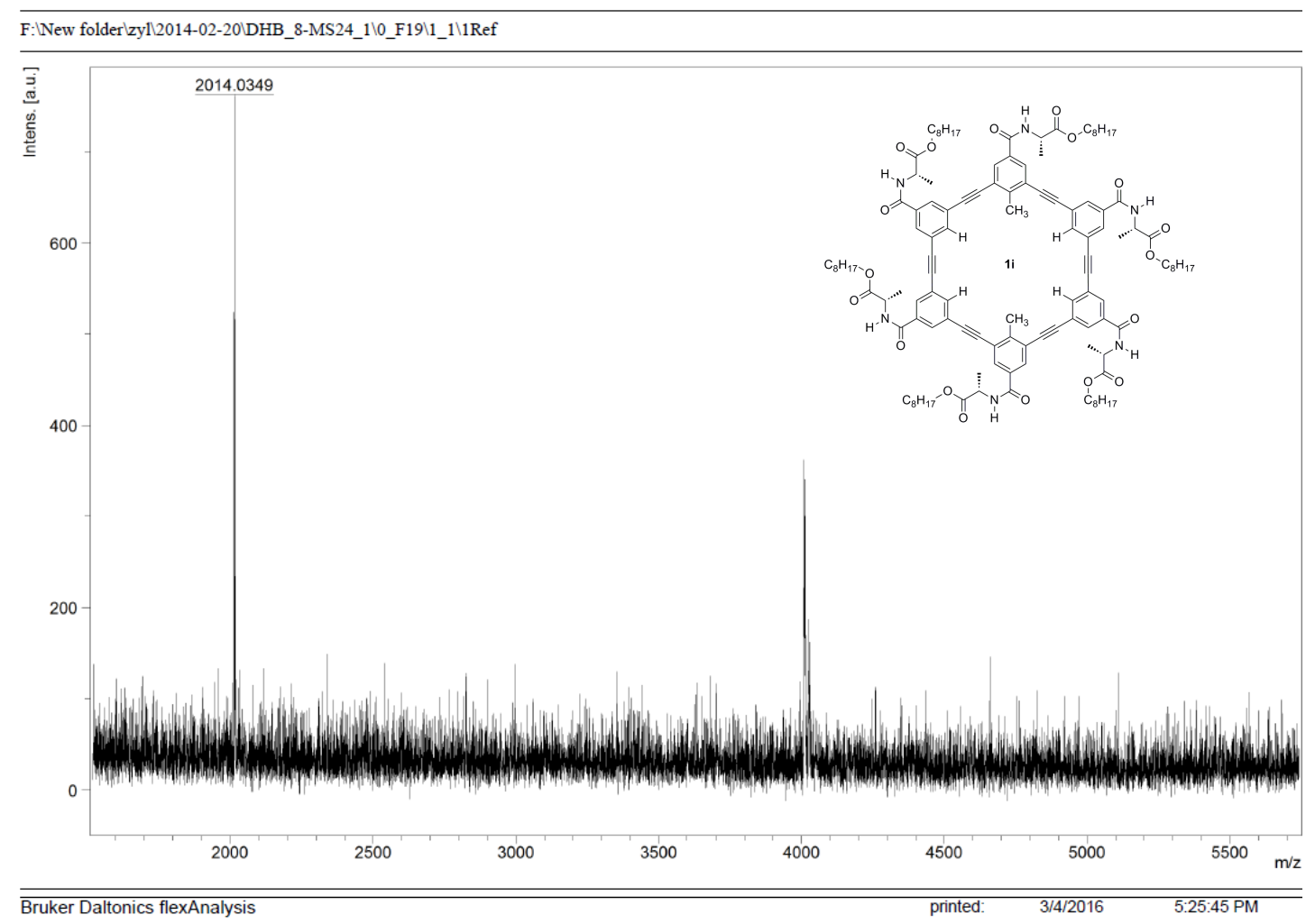

Figure S33Mass Spectra of macrocycle1i

\begin{tabular}{ccccccc}
\hline Formula & Mass & Error & mSigma & DblEq & N rule & $\begin{array}{c}\text { Electron } \\
\text { Configuration }\end{array}$ \\
\hline C 122 H 154 N 6 Na O 18 & $2,014.1212$ & 42.8501 & 682.4064 & 48.50 & ok & even \\
\hline
\end{tabular}




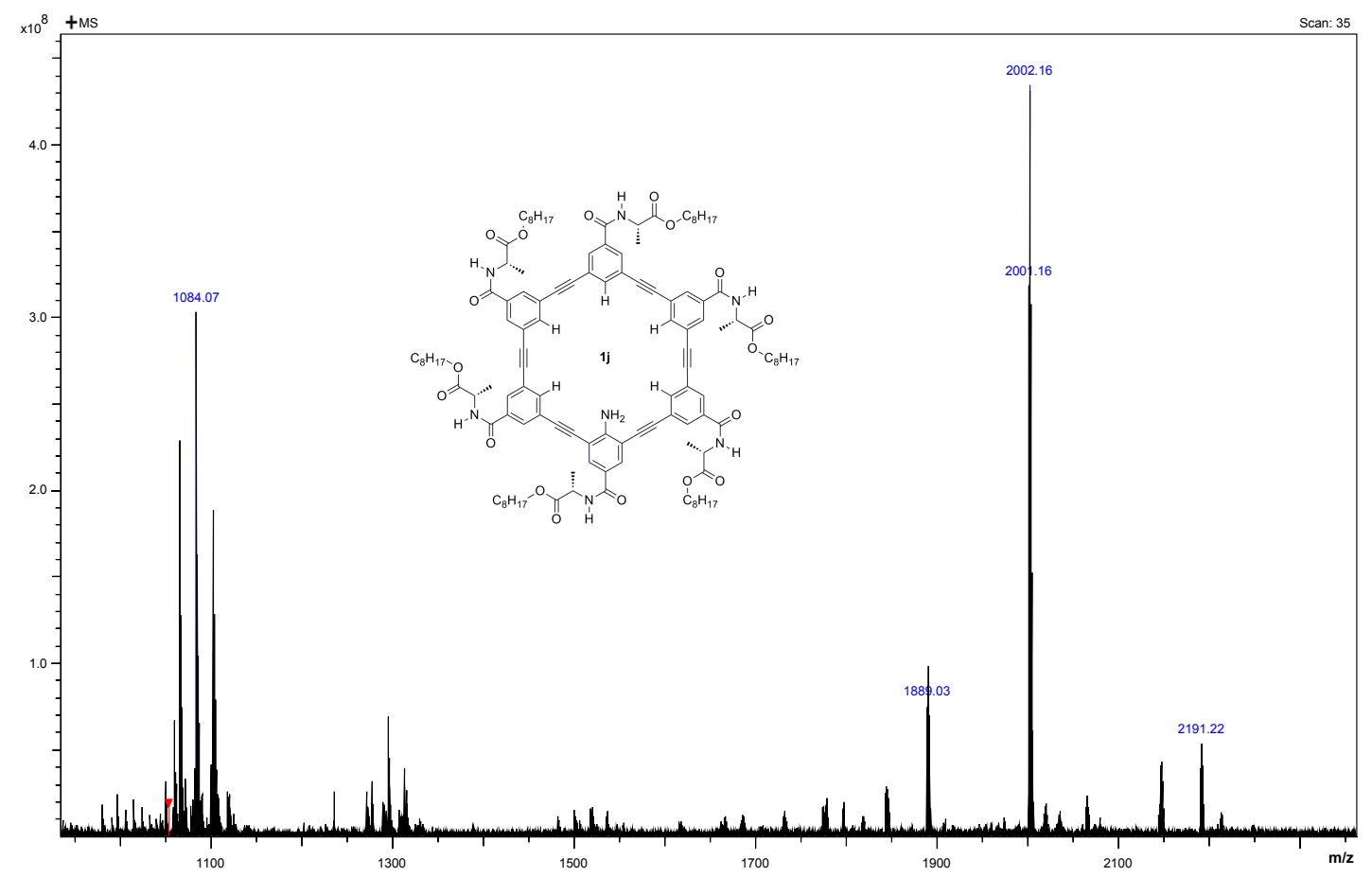

Figure S34Mass Spectra of macrocycle1 $\mathbf{j}$

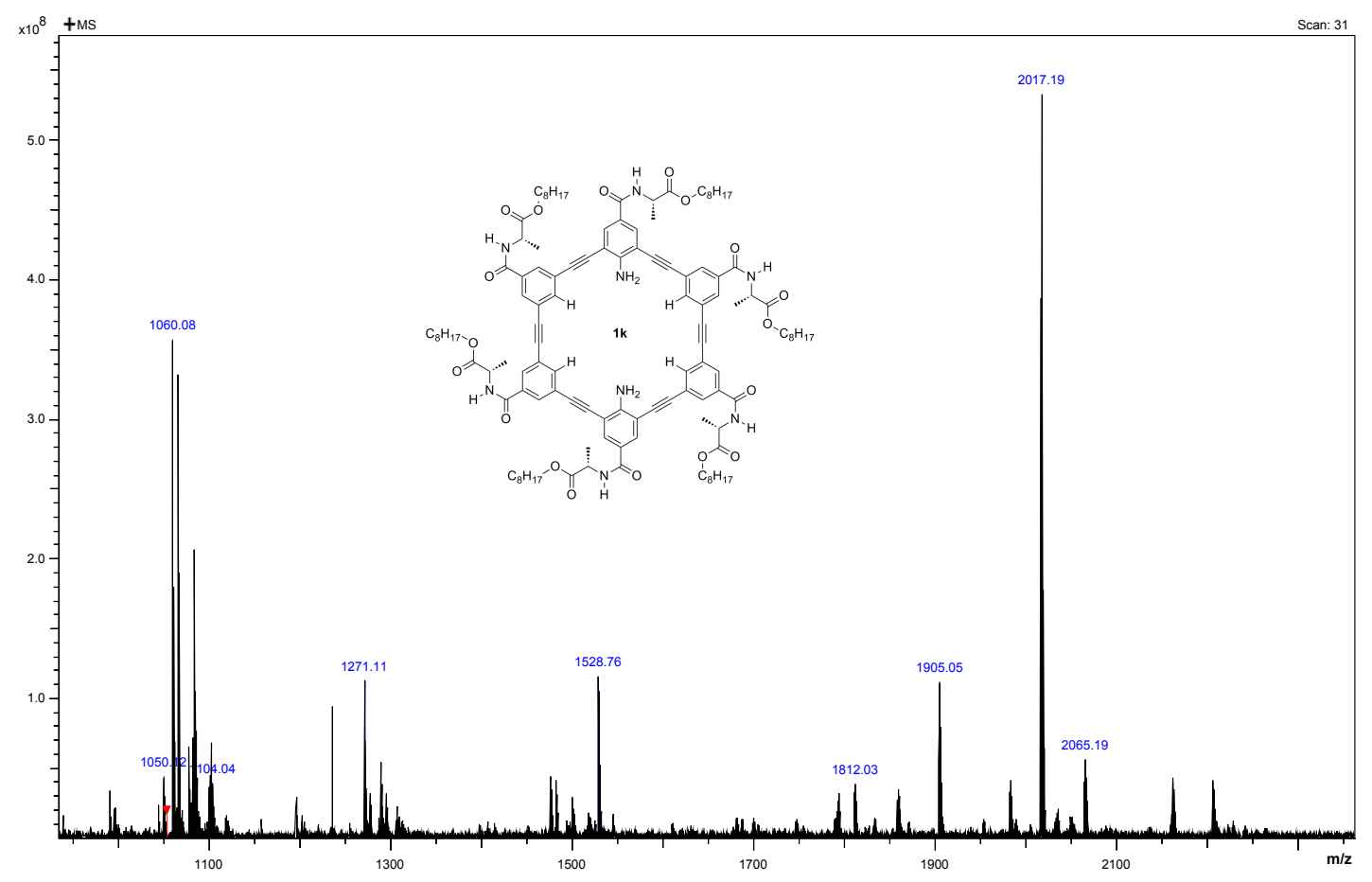

Figure S35Mass Spectra of macrocycle1k 


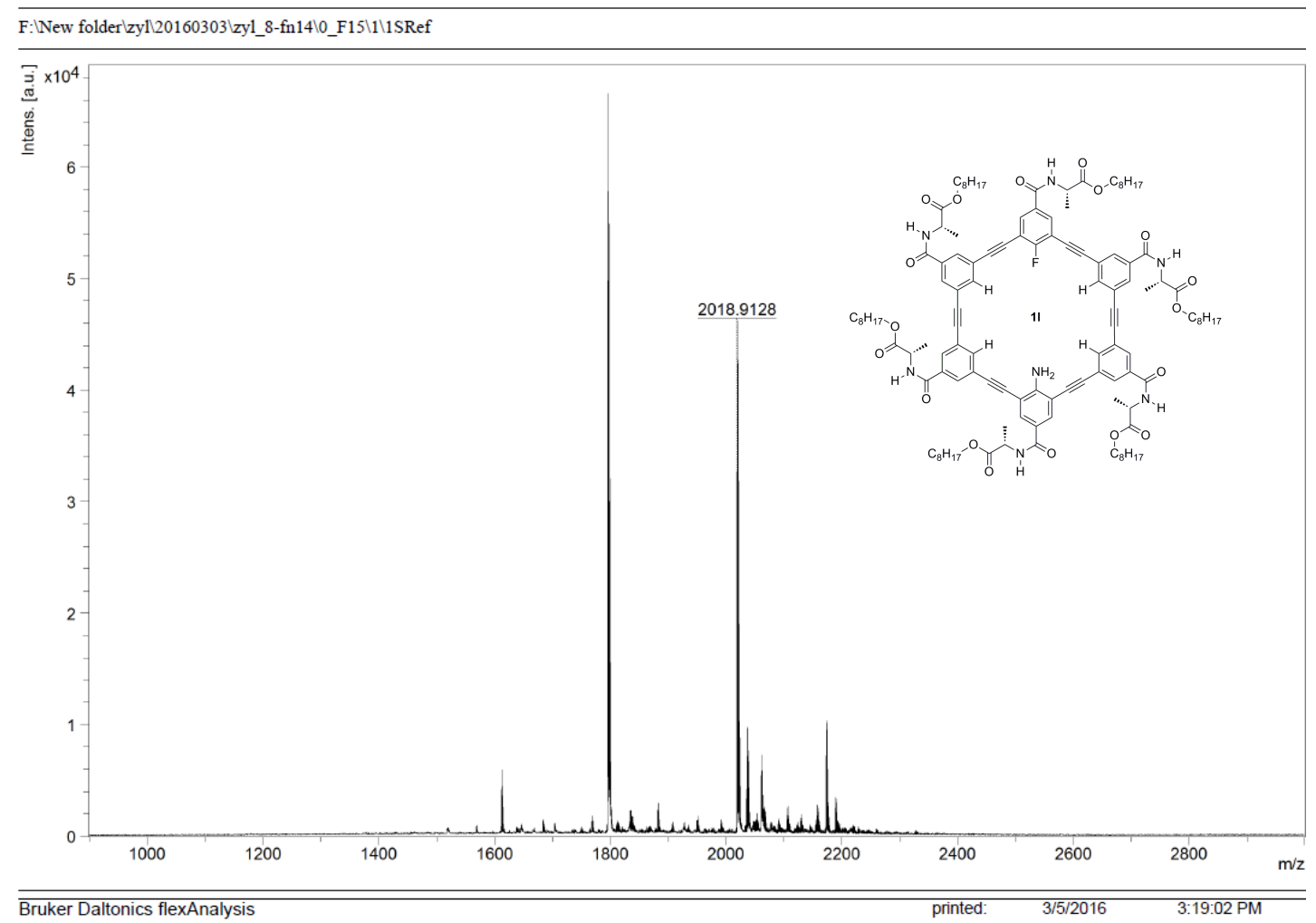

Figure S36Mass Spectra of macrocycle1I

\begin{tabular}{ccccccc}
\hline Formula & Mass & Error & mSigma & DblEq & N rule & $\begin{array}{c}\text { Electron } \\
\text { Configuration }\end{array}$ \\
\hline C 120 H 150 F N 7 Na O 18 & $2,019.0914$ & 88.4464 & 684.8272 & 48.50 & ok & even \\
\hline
\end{tabular}




\section{V. ${ }^{1} \mathrm{H}$ NMR Spectra of Macrocycles $1 \mathrm{a}, 1 \mathrm{c}, 1 \mathrm{~g}, 1 \mathrm{i}, 1 \mathrm{k}$ in $\mathrm{CDCl}_{3}$}

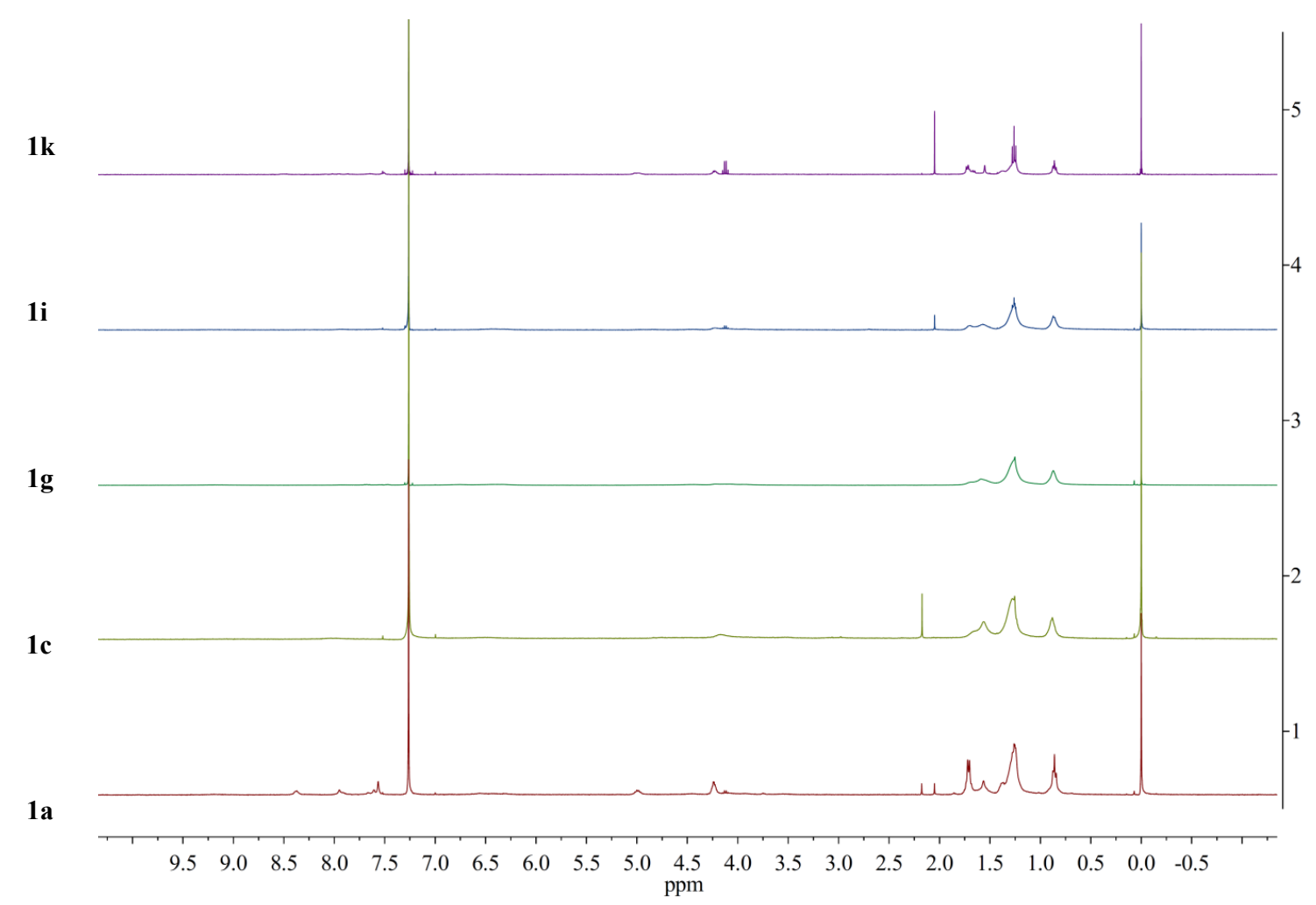

Figure S37 ${ }^{1} \mathrm{H}$ NMR Spectra of $\mathbf{1 a}(1 \mathrm{mM}), \mathbf{1 c}(1 \mathrm{mM}), \mathbf{1 g}(1 \mathrm{mM}), \mathbf{1 i}(1 \mathrm{mM})$, and $\mathbf{1 k}(1 \mathrm{mM})$ in $\mathrm{CDCl}_{3}$ 


\section{UV Spectra of Macrocycles 1a, 1c, 1g, 1i, and 1k}
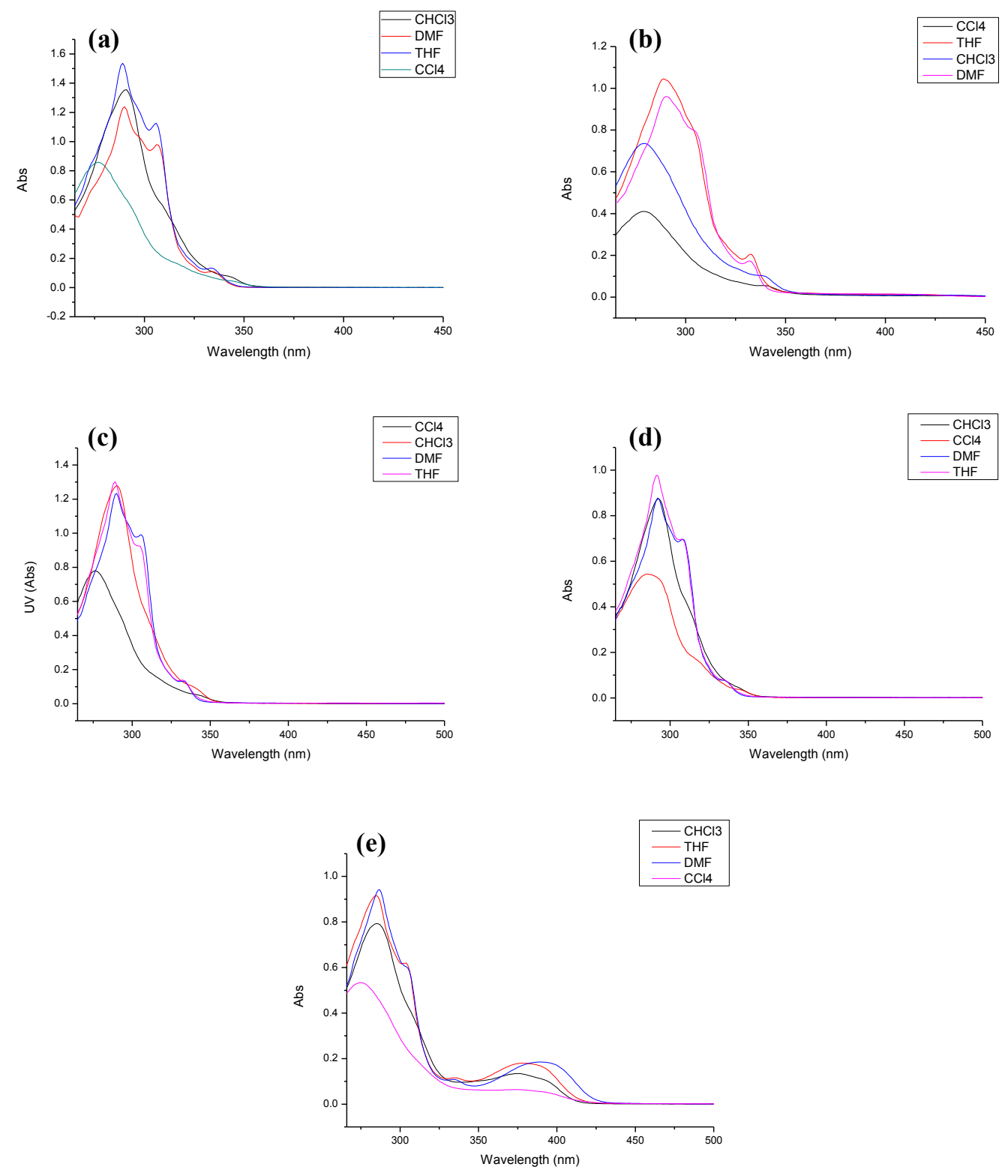

Figure S38UV spectra of (a) 1 a $(5 \mu \mathrm{M})$, (b) $1 \mathrm{c}(5 \mu \mathrm{M})$, (c) 1 g $(5 \mu \mathrm{M})$, (d) $1 \mathbf{i}(5 \mu \mathrm{M})$, and (e) $1 \mathbf{k}$ $(5 \mu \mathrm{M})$ in four different solvents. 


\section{UV Spectra of Macrocycles 1a, 1c, 1g, 1i, and 1k}
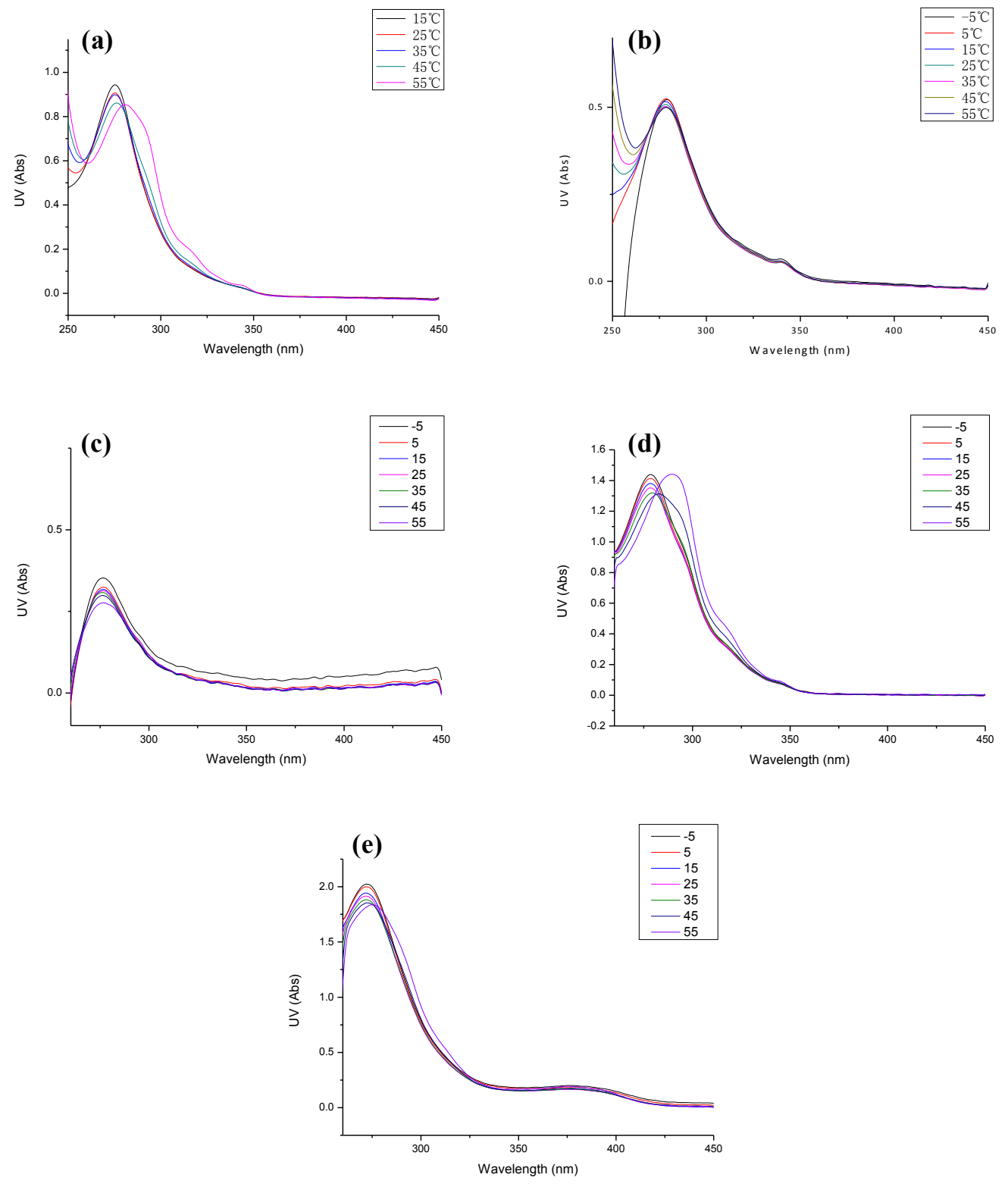

Figure S39Temperature-dependent UV spectra of (a) $\mathbf{1 a}$, (b) $\mathbf{1 c}$, (c) $\mathbf{1 g}$, (d) $\mathbf{1} \mathbf{i}$, and (e) $\mathbf{1 k}$ in $\mathrm{CCl}_{4}$ $(10 \mu \mathrm{M})$. 


\section{CD Spectra of Macrocycles 1a, 1c, 1g, 1i, and 1k}
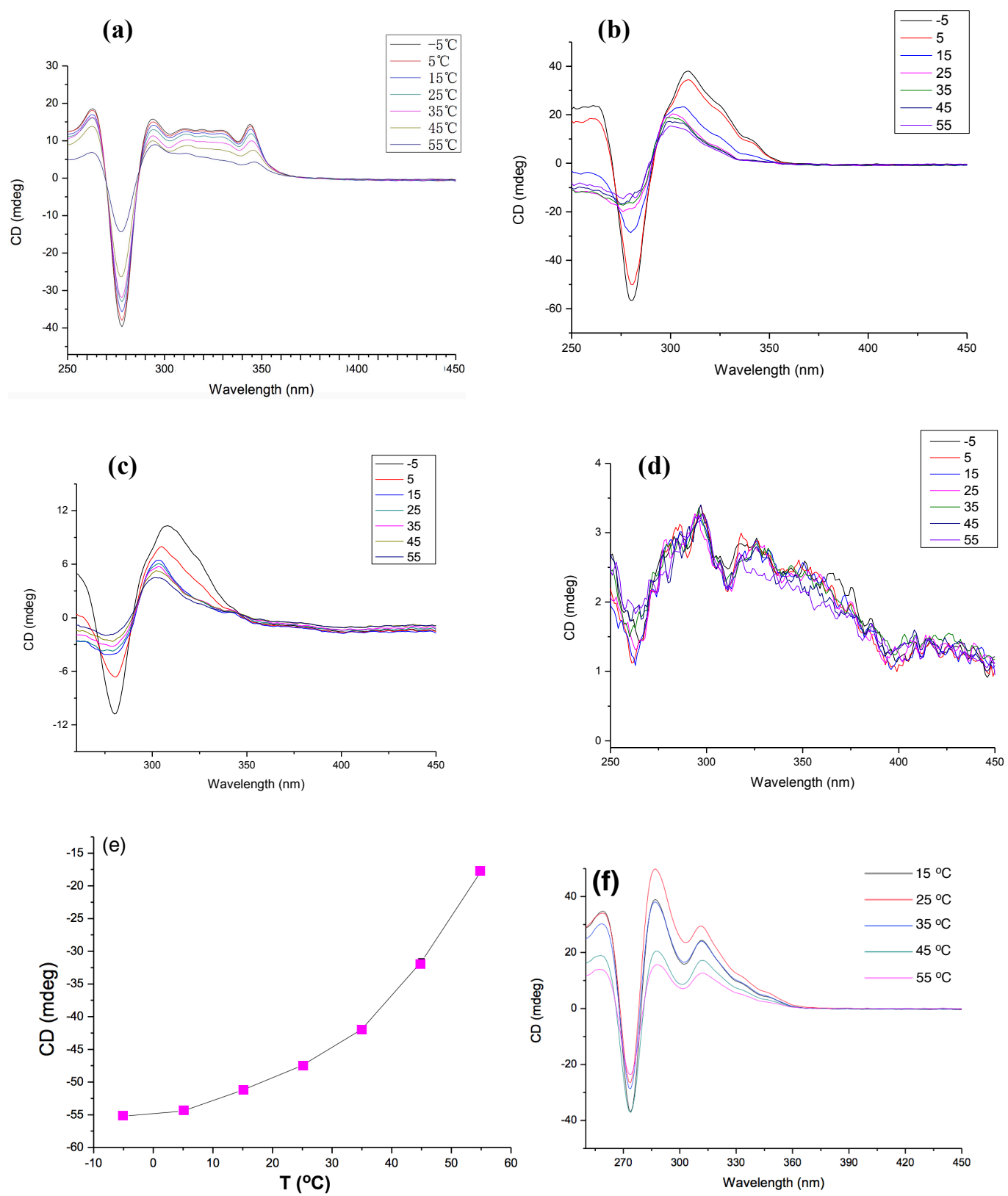

Figure S40Temperature-dependent CD spectra of (a) $1 \mathbf{c}$ in $\mathrm{CHCl}_{3}(10 \mu \mathrm{M})$, (b) $\mathbf{1 g}$ in $\mathrm{CHCl}_{3}$ $(10 \mu \mathrm{M})$, (c) $1 \mathbf{i}$ in $\mathrm{CHCl}_{3}(10 \mu \mathrm{M})$, (d) $\mathbf{1} \mathbf{k}$ in $\mathrm{CHCl}_{3}(10 \mu \mathrm{M})$, (e) plot of the minima of the CD spectra of $1 \mathbf{i}\left(10 \mu \mathrm{M}\right.$ in $\left.\mathrm{CCl}_{4}\right)$ at $277 \mathrm{~nm}$ against temperature, and (f) variable-temperature $\mathrm{CD}$ spectra of 1 a measured in $\mathrm{CCl}_{4}(10 \mu \mathrm{M})$. 